\title{
Manipulation of Monodisperse Emulsions in Microchannels
}

\section{Dissertation}

zur Erlangung des mathematisch-naturwissenschaftlichen Doktorgrades

"Doctor rerum naturalium"

der Georg-August-Universität Göttingen

\section{vorgelegt von \\ Enkhtuul Surenjav}

aus Uws Provinz, Mongolei

Göttingen, 2008 
Referent : Prof. Dr. Stephan Herminghaus

Koreferent : Prof. Dr. Eberhard Bodenschatz

Tag der mündlichen Prüfung : 15. Dezember 2008 


\section{Abstract}

The manipulation of monodisperse gel emulsions confined in a microfluidic channel network has been investigated. Monodisperse gel emulsions were organized by spatial confinement as a function of dispersed phase volume fraction and manipulated using fixed ("passive") and switchable ("active") channel geometries. Furthermore, quasi two-dimensional structural transitions of static emulsion topologies have been studied as a function of lateral force. The controlled droplet formation, targeted electrocoalescence of pairs of droplets, and manipulation of the droplets using the channel geometry has been used to study fibrin network formation and manipulation within the droplet.

Transitions between certain arrangements in an emulsion flowing through a channel can be induced, e.g. by varying the geometry of the channel along its length. Due to the finite energy required to change a certain droplet arrangement, these transitions are inherently hysteretic and depend not only on the droplet size but also on the volume fraction of the dispersed phase. We studied these droplet rearrangements for various channel geometries including constrictions and corners as a function of volume fraction and droplet size. The stability of certain droplet arrangements and their transitions are discussed for static droplet arrangements. We studied the influence of dispersed phase volume fraction and drop size by applying lateral force to the emulsion.

To actively manipulate the emulsion arrangements in a microchannel we used a ferrofluid as the continuous phase of the emulsion. By applying external magnetic fields to the confined arrangement, we could observe a transition between two droplet arrangements. In this case,

a temporarily created ferrofluid plug caused by inhomogeneous magnetic field changes the channel geometry which leads to the transition.

In combination with a technique to coalesce targeted pairs of droplets, we performed in situ measurements of the formation, manipulation, and structure of droplet-encapsulated fibrin networks. In the dynamic case, where droplets were travelling continuously through the 
channels, we observed fibrin network aggregation due to the velocity distribution of the flow field inside the droplet. However, when the droplet is parked in the reaction chamber until the fibrin network is fully developed, then controllably deformed, we observed elastic recovery of the fibrin network. 


\section{Kurzzusammenfassung}

Die Frage wie räumlich auf mikrofluide Kanalnetzwerke begrenzte monodisperse Gelemulsionen auf verschiedene Manipulationen reagieren, wurde in der vorliegenden Arbeit bearbeitet. In verschiedenen Kanalgeometrien wurde die Tropfenanordnung in Abhängigkeit von der Volumenfraktion der dispergierten Phase untersucht, wobei sowohl statische ("passive") als auch schaltbare ("aktive") Kanalgeometrien genutzt wurden. Weiterhin wurden quasizweidimensionale Strukturübergänge an statischen Tropfenanordnungen in Abhängigkeit von der lateral aufgeprägten Kraft betrachtet. Schließlich wurden mehrere Methoden, insbesondere die kontrollierte Tropfenerzeugung, die gezielte Elektrokoaleszenz von Tropfenpaaren und die Manipulation von Tropfen in verschiedenen Kanalgeometrien, angewendet, um die Bildung und Manipulation von Fibrinnetzwerken innerhalb von Tropfen zu studieren.

Übergänge zwischen bestimmten Tropfenanordnungen in einer durch einen Kanal fließenden Emulsion können beispielsweise induziert werden, indem die Kanalgeometrie im Verlauf des Kanals verändert wird. Aufgrund der endlichen Energie, die benötigt wird, um eine bestimmte Tropfenanordnung umzuorganisieren, sind diese Übergänge inhärent hysteretisch und hängen nicht nur von der Tropfengröße, sondern auch vom Volumenanteil der dispergierten Phase ab. Es wurden die Tropfenumordnungen für verschiedene Kanalgeometrien, wie Einengungen und Ecken, abhängig vom Volumenanteil und Tropfengröße studiert. Des Weiteren war auch der Einfluss der Volumenfraktion und der Tropfengröße auf Übergänge in der Tropfenanordnung bei Einwirkung einer lateralen Kraft Gegenstand der Arbeit.

Um die Emulsionsanordnungen in einem Mikrokanal aktiv manipulieren zu können wurden Ferrofluide als kontinuierliche Phase der Emulsion verwendet. Durch das Anlegen von externen Magnetfeldern an die geschlossene Anordnung konnten Übergänge zwischen zwei Tropfenanordnungen beobachtet werden. Dabei wurden vorübergehend ferrofluidische Ansammlungen hergestellt, die die Kanalgeometrie so veränderten, dass Strukturübergänge zu beobachten waren. 
In Kombination mit einer Technik, bei der gezielt Paare von Tropfen fusioniert werden, wurde die Bildung und Manipulation in situ sowie die Struktur von in Tropfen eingeschlossenen Fibrinnetzwerken untersucht. Im dynamischen Fall, bei dem sich Tropfen kontinuierlich durch die Kanäle bewegen, wurde die Aggregation von Fibrinnetzwerken in Abhängigkeit von der Geschwindigkeitsverteilung im Strömungsfeld innerhalb des Tropfens beobachtet. Wurde der Tropfen aber in der Reaktionskammer bis zur vollständigen Entwicklung des Fibrinnetzwerkes "geparkt" und dann kontrolliert deformiert, wurde anders als im dynamischen Fall eine elastische Wiederherstellung der ursprünglichen Form des Fibrinnetzwerkes beobachtet. 


\section{Contents}

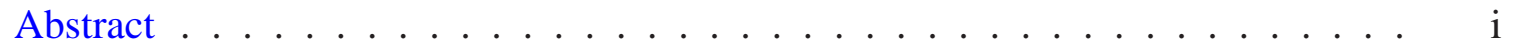

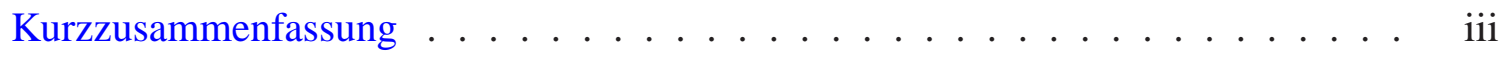

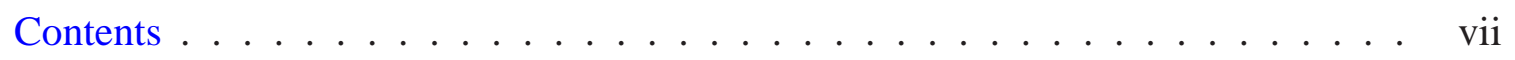

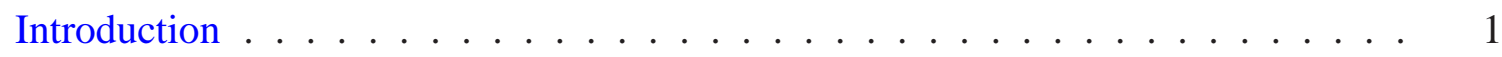

1 Background $\quad 5$

1.1 Physics of liquids at small length scales $\ldots \ldots \ldots \ldots$

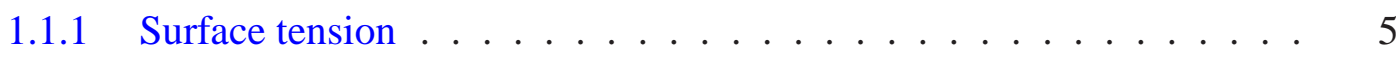

1.1 .2 Pendant drop method . . . . . . . . . . . . . . . 6

1.1 .3 Reynolds number . . . . . . . . . . . . . . 7

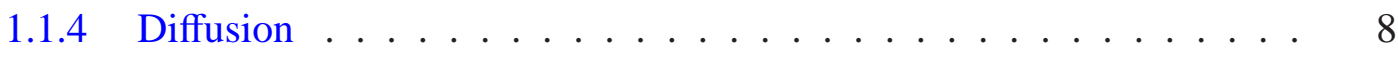

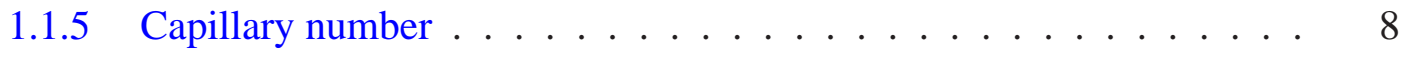

1.2 Emulsification . . . . . . . . . . . . . . . . 9

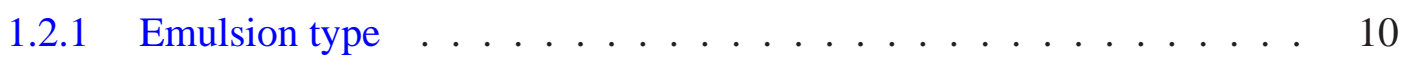

1.2 .2 Emulsion production $\ldots \ldots \ldots \ldots \ldots \ldots \ldots \ldots$

1.2 .3 Emulsion stability $\ldots \ldots \ldots \ldots \ldots$

1.3 Foams . . . . . . . . . . . . . . . . . . . . . . . . 14

1.3.1 Laws of Plateau . . . . . . . . . . . . . . . . . . . 14

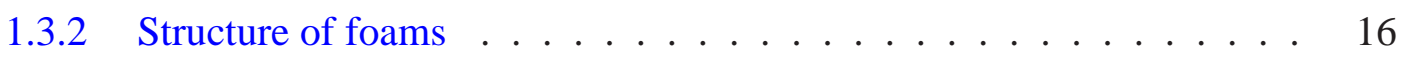

2 Materials, Methods and Experimental Techniques 17

2.1 Emulsion systems . . . . . . . . . . . . . . . . . . . . . . . 17

2.2 Microchannel fabrication . . . . . . . . . . . . . . . . . . . . . 19

2.2.1 Micromachined channels . . . . . . . . . . . . . . . . . 20

2.2.2 Photolithography channels . . . . . . . . . . . . . . . 20

2.3 Experimental set-up . . . . . . . . . . . . . . . . . . 23

2.4 Monodisperse emulsion production in microchannels . . . . . . . . . . . 24 
2.4.1 T-junction emulsification . . . . . . . . . . . . . . . 24

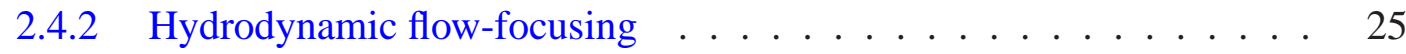

2.4 .3 Step emulsification . . . . . . . . . . . . . . 26

2.4.4 Manipulating the volume fraction of the emulsion after production . . 32

2.5 Droplet coalescence . . . . . . . . . . . . . . . . . . . 33

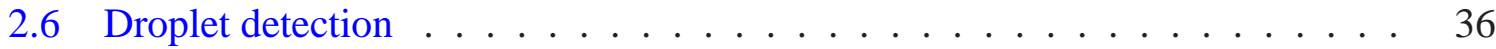

3 Manipulation by Channel Geometry 39

3.1 Structural transitions . . . . . . . . . . . . . . . . . . 40

3.2 Droplet reorganizations at a corner . . . . . . . . . . . . 47

4 Manipulation by Magnetic Fields

4.1 Water-in-ferrofluid emulsions in capillaries . . . . . . . . . . . . . 56

4.2 Water-in-ferrofluid emulsions in microchannels . . . . . . . . . . . 58

5 2D Structural Transitions of a Static Droplet Arrangements 67

5.1 Device design and experimental conditions _. . . . . . . . . . 68

5.2 Results and discussion . . . . . . . . . . . . . . . . . . 70

6 Formation and Manipulation of Droplet-Encapsulated Fibrin Network 75

6.1 Fibrin . . . . . . . . . . . . . . . . . . . . 75

6.2 Experiments . . . . . . . . . . . . . . . . . . . 77

6.3 Fibrin network clotting in droplets . . . . . . . . . . . . . 78

6.4 Manipulation of fibrin network . . . . . . . . . . . . . 81

6.5 Coacervation of deformed fibrin networks . . . . . . . . . . . 87

$\begin{array}{llr}7 & \text { Summary } & 91\end{array}$

$\begin{array}{lr}\text { Appendices } & 94\end{array}$

$\begin{array}{llr}\text { A Symbols and notations } & 97\end{array}$

B Recipe for photolithographic devices $\quad 99$

B.1 Underlayer . . . . . . . . . . . . . . . . . . . . . . . . . 99

B.2 Channel structures . . . . . . . . . . . . . . . . . . . . . 99

B.2.1 Resist Structure: $30 \mu \mathrm{m}$ channel height . . . . . . . . . . . . 99

B.2.2 Resist Structure: $50 \mu \mathrm{m}$ channel height . . . . . . . . . . 100 
B.2.3 Resist Structure: $80 \mu \mathrm{m}$ channel height . . . . . . . . . . . 100

B.2.4 Resist Structure: $120 \mu \mathrm{m}$ channel height . . . . . . . . . . . . 100

B.3 Preparation of PMMA coated cover plate . . . . . . . . . . . . 101

B.4 Thermal bonding of the channels and the cover plate . . . . . . . . . . 101

B.5 Nanoport bonding . . . . . . . . . . . . . . . . . . . . . . 101

$\begin{array}{ll}\text { References } & 103\end{array}$

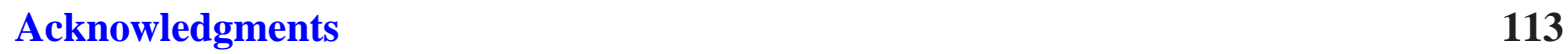





\section{Introduction}

Microfluidics is a rapidly growing field, in which fluids are manipulated with precise control at a micro- or even nanoscopic scale. Microfluidic technology holds great promise as it can perform typical laboratory operations using a fraction of the volume of reagents in significantly less time than compared with conventional batch experiments. In the classical concept of microfluidic devices, fluids are manipulated under continuous flow through microfabricated channels [1]. Alternatively, droplet-based ("discrete") microfluidic systems can handle and manipulate individual droplets where microfluidic processing is performed on monodispersed aliquots which are transported, stored, mixed, reacted, or analyzed in a discrete manner [2$10]$.

There are several advantages regarding the use of discrete microfluidics when compared with continuous flow systems, including precise control of sample volumes (droplets), and high throughput productivity and high integration with other microchip components [11]. Another advantage of this approach is the flow pattern emerging within the droplets as they travel through the microchannel which can be used to mix the droplet's contents [12-14]. Due to the low Reynolds numbers at small scales, mixing is rather slow for microflow and therefore proceeds via diffusion, viscous dephasing [15], or requires special microchannel designs to facilitate "quasi chaotic mixing" [16-21]. In droplet-based microfluidics, mixing can be achieved within individual droplets and is found to proceed quite efficiently [22-26]. A relatively new branch of research is focused on loading each droplet with different chemical or biological species which could be used as a reactor for combinatorial chemistry. One way to perform re-

actions in droplet-based microfluidic systems is by bringing reagents together in a co-flowing stream immediately before droplet formation or by using a side channel; the reaction occurs downstream in the two component microdroplet [22, 23, 27-30]. However, this method is unsuitable for aggressive or fast reagents which adhere to the channel walls [31]. To prevent sticky interaction between the reagents and channel surface and to induce well controlled 
reactions, it is necessary to initiate the reaction by fusion of two droplets, each containing different reagents. Alternatively, a cartridge could be loaded by "conventional pipetting" and the different chemical contents fed ex-situ from a pipette into the microfluidic device [32-35]. Furthermore, the ability to exchange the relative positions of specific droplets and to merge droplets on demand [36] to initiate the reaction may be possible. This might enables the initiation of chemical reactions in a combinatorial way [37-39], as well as handling of discrete composition variations for high-throughput screening purposes. To date, much research has been dedicated to the fundamental aspects of droplet-based microfluidics, including droplet formation [40-45], detection [46, 47] manipulation [48-51], and coalescence [36, 38, 52-54].

Microfluidic technology has been successfully applied for numerous biological and biochemical applications. One promising application is for DNA amplification using the polymerase chain reaction (PCR). PCR is a common procedure in molecular biology for generic analysis [55]. During the PCR procedure, the concentration of a certain segment of doublestranded DNA is doubled through a thermal cycling process involving three different temperatures. Encapsulation of cells within the droplet has also been successfully investigated by a number of groups $[31,56,57]$. Furthermore, the use of microfluidic tools enables investigations of protein crystallization $[25,58]$, the synthesis of particles [59, 60] and the assay of biological entities [61-63]. Zheng et al. [29] have demonstrated protein screening in droplets using parallel stream flows, and Hirano et al. [64] have obtained successful crystal growth in mixed droplets.

This thesis focuses on the microfluidic flow behaviour of a densely packed arrangement of droplets in a continuous phase. At high dispersed phase volume fraction, the monodisperse compartments (droplets) of an emulsion assemble into well-ordered arrangements while being transported along microfluidic channels. We studied the organization and manipulation of monodisperse gel emulsions as a function of microchannel geometries, droplet sizes, and dispersed phase volume fractions, which are directly transferable to integrated microfluidic devices. A technique to coalesce and manipulate the individual droplets has been combined to investigate an encapsulated fibrin network formation. This thesis consists of six chapters and is organized as follows: The general background includes the physics of fluids at small length scales, emulsification and foaming will be discussed in the first chapter. In the second chapter the materials, methods and experimental techniques used in this thesis will be outlined. The structural transition of droplet arrangements by the channel geometry will be presented in chapter three. Here, we explore the parameters that define droplet arrangements and investigate switching behavior between different arrangements. The active manipulation of emulsion 
arrangements by magnetic field where ferrofluid is used as the continuous phase will be presented in chapter four. In chapter five, we will discuss a stability of droplet arrangements and their transition as a function of an applied lateral force. As an application of droplet-based microfluidics, we investigated affine aspects of the fibrin network formation and its behavior within a droplet which will be presented in the final chapter. 



\section{Chapter 1}

\section{Background}

\subsection{Physics of liquids at small length scales}

In order to understand and work with microfluidics, one must first understand the physical phenomena that dominate the liquid flow at the microscale. The characteristics of fluids at the microscale differ from 'macrofluidic' behavior. The surface energies are dominant over the effect of gravity at small length scale. The typical Reynold's number of the flow is very small at small length scale of a typically used microchannels, therefore flow is laminar. Thus, mixing of two liquids at laminar flow proceeds predominantly by diffusion.

\subsubsection{Surface tension}

Surface tension, $\gamma$, between two immiscible phases is caused by the attraction between the molecules of the liquid by intermolecular forces. In the bulk of the liquid, each molecule is pulled equally in all directions by neighboring liquid molecules, resulting in a zero net force. At the surface of the liquid, the molecules are pulled inwards by other molecules deeper inside the liquid and are not attracted as much by the molecules in the neighboring medium (be it vacuum, air or another liquid). Therefore, the molecules at the surface are subject to an inward force of molecular attraction which can be balanced only by the resistance of the liquid to compression. This inward attraction tends to minimize the surface area, and in this respect a liquid surface resembles a stretched elastic membrane. Thus the liquid will always minimize its interfacial area.

Because of the existence of surface tension effects, there will be a pressure difference across any curved liquid surface, with the pressure larger on the concave side of the interface. 
That means, the pressure inside a bubble/droplet will always be greater than that in the continuous phase. The magnitude of this pressure differential is given by the Young - Laplace equation:

$$
\Delta p=\gamma\left(\frac{1}{r_{1}}+\frac{1}{r_{2}}\right)
$$

where $\gamma$ is the surface tension of the respective liquid interface and $r_{1}$ and $r_{2}$ are the two principal radii of the curved interface. If the surface is spherical, i.e. $r_{1}=r_{2}$, then Eq. 1.1 reduces to

$$
\Delta p=\frac{2 \gamma}{r}
$$

and allows the calculation of the pressure contained within a free droplet or bubble in another phase. In case of soap bubbles, Eq. 1.2 must be adjusted to be

$$
\Delta p=\frac{4 \gamma}{r}
$$

due to the two liquid-vapour interfaces involved.

\subsubsection{Pendant drop method}

There exists a number of different methods to measure the surface tension of liquid/air or liquid/liquid interfaces. Which method is preferred depends upon the nature of the liquid being measured, the conditions under which the surface tension is to be measured, and the stability of its surface when it is deformed. Drop shape analysis at a capillary is a convenient way to measure surface tension. There are two principal assumptions that one has to take into account to get a reliable result. First, the drop has to be symmetric about a central vertical axis; this means it is irrelevant from which direction the drop is viewed. Second, the drop is not in motion in the sense that viscosity or inertia are playing a role in determining its shape; this means that surface tension and gravity are the only forces shaping the drop.

The shape of a drop of a certain liquid hanging from a syringe tip (see Fig. 1.1) is determined from the balance of forces which include the surface tension of the liquid. The surrounding phase can be air or liquid. The surface or interfacial tension at the liquid interface 


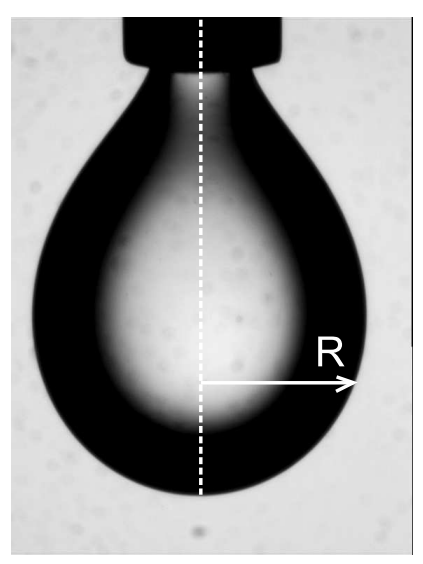

Figure 1.1: Image of a pendant drop.

can be related to the drop shape through the following equation [65]:

$$
\gamma=\Delta \rho g R^{2} / \beta
$$

where $\gamma$ is the interfacial tension between the liquids, $\rho$ is the density difference between fluids at interface, $g$ is the gravitational constant, $R$ is the radii of drop curvature at the apex (cf. Fig. 1.1 ) and $\beta$ is the shape factor of the drop which determines the dimensionless drop shape profile. Thus for any pendant drop where the densities of the two fluids in contact are known, the surface tension may be measured based upon the Eq. 1.4. This approach represents a significant improvement, in both ease and accuracy, from traditional methods e.g. Wilhelmy plate method. This method has advantages in that it is able to use very small volumes of liquid and measure very low interfacial tensions.

\subsubsection{Reynolds number}

The Reynolds number is a measure of the ratio of inertial forces to viscous forces and therefore, is a measure of the relative importance of these two forces under given flow conditions. Thus it can be used to identify and predict different flow regimes, such as laminar or turbulent flow. Laminar flow occurs at low Reynolds numbers, where viscous forces are dominant, and is characterized by smooth, constant fluid motion, while turbulent flow, on the other hand, occurs at high Reynolds numbers and is dominated by inertial forces, which tend to produce 
random eddies, vortices and other flow fluctuations. The Reynolds number is given by

$$
R e=\frac{\rho v l}{\eta}
$$

where $\rho$ is the fluid density, $v$ is the characteristic velocity of the fluid, $\eta$ is the fluid viscosity, and $l$ is a characteristic length. $R e \ll 2000$, as calculated by Eq. 1.5 generally indicates a laminar flow. As Re approaches 2000, the fluid begins to show signs of turbulence, and as $R e$ becomes much larger than 2000 the flow is considered to be turbulent. Because of the small size of microchannels the Reynolds number in microfluidic system hardly exceeds unity and thus the flow is almost always laminar. One consequence of the laminar flow is that two or more streams flowing in contact with each other will not mix except by diffusion.

\subsubsection{Diffusion}

Diffusion is the process by which molecules spread from regions of higher concentration, to regions of lower concentration. Diffusion can be described in one dimension by the equation $d^{2}=2 D t$, where $d$ is the distance a particle spreads in a time $t$, and $D$ is the diffusion coefficient of the particle. Due to the typical length scale of microfluidic devices, the time for this diffusive mixing often exceeds processing times for other steps, e.g. transport and analysis. For instance, small solutes with diffusion coefficients on the order of $D=10^{-5} \mathrm{~cm}^{2} / \mathrm{s}$, mixing by diffusion across a $500 \mu \mathrm{m}$ wide channel would result in a mixing time of several minutes. For larger solutes such as proteins $\left(D \sim 10^{-7} \mathrm{~cm}^{2} / \mathrm{s}\right)$, the time needed for diffusive mixing becomes extremely long. This slow time scale may be a hindrance for high-throughput microfluidic applications. However, in droplet-based microfluidics, mixing can be achieved within individual droplets, and is found to proceed quite efficiently where special channel geometries are used [22-26] as we will discuss later.

\subsubsection{Capillary number}

The dimensionless capillary number, $C a$, plays a key role in determining the dynamics, such as fission or droplet break-up. The capillary number represents the ratio of viscous forces versus surface tension acting across an interface between a liquid and a gas, or between two immiscible liquids. It is defined as: $C a=\eta v / \gamma$, where $\eta$ is the viscosity of the continuous phase, $v$ is the velocity of the continuous phase, and $\gamma$ is the interfacial tension between the 
two liquid phases. In complex geometries such as microfluidic devices it is more convenient to define capillary number through externally controlled parameters like flow rate and viscosity of two phases involved and device geometry. Above a certain critical capillary number, droplet break-up occurs. To realize this number, it is important to consider the relative viscosity between the dispersed and the continuous phases. Selection of a more viscous continuous phase will facilitate formation of droplets. For the formation of water-in-oil (W/O) emulsions, the continuous phase consists of water immiscible organic solvents, which are naturally more viscous than water. In the case of oil-in-water $(\mathrm{O} / \mathrm{W})$ emulsions, the addition of viscous watermiscible fluids such as glycerol into the aqueous continuous phase improves shearing of the more viscous oily dispersed phase to produce droplets.

\subsection{Emulsification}

Two immiscible liquids cannot form a stable emulsion. For a suspension of one liquid in another to be stable enough to be classified as an emulsion, typically a third component must be present to stabilize the system. The third component is a surface active agent (abbreviated to "surfactant") that reduces the interfacial tension between the two liquids by adsorbing at the liquid-liquid interface as an oriented interfacial film and stabilizes the droplets, cf. Fig. 1.2 a. The surfactant's chemical structure consists of a hydrophobic tail which is usually a hydrocarbon, although also fluorocarbon and dimethylsiloxane chains can be used, with a polar hydrophilic head group which may be ionic or non-ionic.

Depending on the size of the droplet, an emulsion is classified into three different group namely macroemulsion, mini-emulsion and microemulsion. A macroemulsion is thermodynamically unstable but can be kinetically stabilized by surfactant and the droplet sizes typically range from a few to hundreds of microns. Using co-surfactant/surfactant systems droplet sizes down to $\sim 100 \mathrm{~nm}$ may be produced and the resulting emulsions are referred to as 'miniemulsions' [66]. Co-surfactants are added to increase the stability of mini-emulsions and must be soluble in the dispersed phase. Another term for co-surfactant is costabilizer because of cosurfactant has no surface active properties. The role of the costabilizer, as the name suggests, is to act together with the surfactant to provide stability to the droplets, in this case, stability against Ostwald ripening [67] (see also section 1.2.2). If interfacial tensions are reduced to very low levels though due to the larger amount of surfactant, even emulsions with a droplet sizes down to $\sim 10 \mathrm{~nm}$ can be produced and the emulsion appears transparent due to the small 
size of the dispersed phase droplets. The viscosity is usually low, unlike liquid crystal phases, and the stability is quite different from what we regard as 'normal emulsions'. Such systems represent thermodynamically stable phases and are termed microemulsions [68]. The properties of microemulsions are determined by the properties of the surfactant monolayer, separating the oil from the water domains.

a) Surfactant stabilizing emulsion

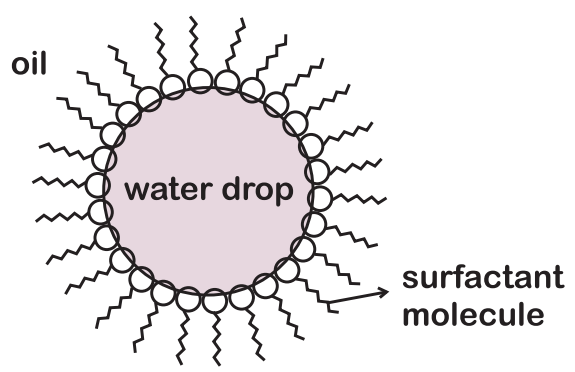

b) Pickering emulsion

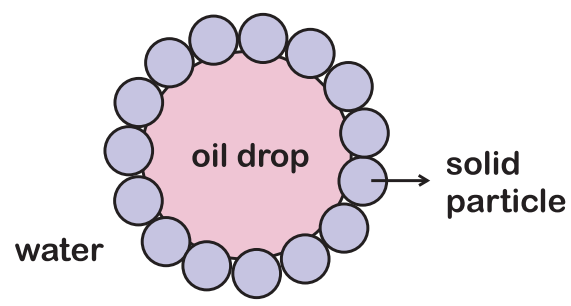

Figure 1.2: A sketch of a) W/O emulsion stabilized by surfactant, b) $\mathrm{O} / \mathrm{W}$ emulsion stabilized by solid particles.

When an emulsion is stabilized using small solid particles, which adsorb onto the interface between the two phases, it is called a Pickering emulsion [69], cf. Fig. 1.2 b. Properties such as hydrophobicity, shape, size and concentration of the particles can have an effect on the stability of the emulsion. Particles that are partially hydrophobic (i.e. contact angle of approximately $90^{\circ}$ ) are better stabilizers because they are partially wettable by both liquids and therefore bind better to the surface of the droplets.

\subsubsection{Emulsion type}

The nature of the surfactant can determine the type of the emulsion, i.e. which liquid will be dispersed as droplets and which will form the continuous phase. For this there are several empirical predictive approaches based on the assumed surfactant positioning at the interface, including Bancroft's rule [70], the oriented wedge theory (which can also be applied to fine solids), the hydrophile - lipophile balance (HLB) [71], and the volume balance value [72]. Although there are exceptions to each of these rules, they remain useful for making initial predictions. The surfactants which are more soluble in water tend to make oil-in-water $(\mathrm{O} / \mathrm{W})$ emulsions and surfactants more soluble in oil tend to make water-in-oil (W/O) emulsions. This is the essence of Bancroft's rule which states that the continuous phase of an emulsion will 
be the phase in which the surfactant is preferentially soluble [70]. Thus it was long known that the balance between hydrophilic and lipophilic (hydrophobic) moieties greatly influences the emulsion type. However, there was no quantitative measure of this balance until 1949, when Griffin introduced the concept of the HLB, the hydrophile-lipophile balance, as a way of predicting the emulsion type from the molecular composition of the surfactant [71]. The HLB number is defined in terms of numerical values assigned to the chemical groupings in the surfactant, as follows:

$$
\left.H L B=7+\sum \text { (hydrophilic group numbers }\right)-\sum(\text { lipophilic group numbers })
$$

\begin{tabular}{c|c|c}
\hline Type & Chemical group & Group number \\
\hline \hline Lypophilic & $-\mathrm{CH}-$ & 0.475 \\
& $=\mathrm{CH}-$ & 0.475 \\
& $-\mathrm{CH}_{2}-$ & 0.475 \\
& $\mathrm{CH}_{3}-$ & 0.475 \\
Hydrophilic & $-\mathrm{SO}_{4} \mathrm{Na}$ & 38.7 \\
& $-\mathrm{COOK}$ & 21.1 \\
& $-\mathrm{COONa}_{7}$ & 19.1 \\
& $-\mathrm{SO}_{3} \mathrm{Na}$ & 11.0 \\
& $=\mathrm{N}-$ & 9.4 \\
& Ester (sorbitan ring) & 6.8 \\
& Ester (free) & 2.4 \\
& $-\mathrm{COOH}$ & 2.1 \\
& $-\mathrm{OH}$ (free) & 1.9 \\
& $-\mathrm{O}-$ & 1.3 \\
& $-\mathrm{OH}$ (sorbitan ring) & 0.5
\end{tabular}

Table 1.1: HLB numbers for various chemical groups, taken from reference [68].

The group numbers assigned by Davies and Rideal [73] are given in Table 1.1. This dimensionless scale ranges from $0-20$ for non-ionic surfactants; a low HLB number indicates a more hydrophobic surfactant and a high HLB number indicates a more hydrophilic surfactant. Thus, W/O and O/W emulsions are favored for low and high HLB numbers, respectively. Most ionic surfactants have HLB values greater than 20, for example, sodium dodecyl sulfate (SDS) has an HLB of 40. 
Just as solubilities of surfactants vary with temperature, so does the HLB, especially for the non-ionic surfactants. A surfactant may thus stabilize $\mathrm{O} / \mathrm{W}$ emulsions at low temperature, but $\mathrm{W} / \mathrm{O}$ emulsions at some higher temperature. The transition temperature, at which the surfactant changes from stabilizing $\mathrm{O} / \mathrm{W}$ to $\mathrm{W} / \mathrm{O}$ emulsions, is known as the phase inversion temperature, PIT. At the PIT, the hydrophilic and lipophilic natures of the surfactant are essentially the same (another term for this is the HLB temperature). As a practical matter, surfactants are chosen so that their PIT is far from the expected storage and use temperatures of the desired emulsions.

\subsubsection{Emulsion production}

Techniques used to produce emulsions in a laboratory include membrane extrusion [74, 75], micro-thread generation [76], viscoelastic shear [77, 78] and microchannel emulsification [40$45,79]$. In this thesis a microchannel emulsification technique is used to produce an emulsion. A detailed description of droplet production in a microchannel which are used in framework of this thesis will be discussed in chapter 2, under the section called 'Monodisperse emulsion production in microchannels'. When microfabricated channels are used to create an emulsion, the wetting competition of fluids for the channel walls (which fluid preferentially interacts with the channel surfaces) [80], will also determine the arrangement of the phases. Hydrophobic channels are needed to generate water-in-oil droplets, in which the water phase is sheared by oil streams, whereas hydrophilic channels are necessary for oil-in-water emulsion generation. By changing the geometry and the wettability of the microchannel it is possible to produce a double emulsion [81]. A double emulsion (also referred to as a multiple emulsion) can be defined as a multiple-phase dispersion in which droplets enclosing finer droplets are suspended in a continuous liquid phase, cf. Fig. 1.3. A microfluidic device having a hydrophobic junction and a hydrophilic junction positioned in series has been used to generate a water-in-oil-in-water (W/O/W) emulsion [82]. For producing $\mathrm{W} / \mathrm{O} / \mathrm{W}$ dispersions, aqueous droplets formed at the upstream hydrophobic junction are enclosed within oil droplets formed at the downstream hydrophilic junction. Utada et al. fabricated a microcapillary device which can be used to produce a double emulsion that contained a single internal droplet in a coreshell geometry [83]. In principle, the size, the number, and even the composition of the drops can be controlled at each step of the process, leading to a unique way of producing monodisperse, perfectly controlled, multiple emulsions. Recently a review paper by Nisisako showed a way to produce multiple emulsions by various emulsification methods [84]. Both W/O/W and 
$\mathrm{O} / \mathrm{W} / \mathrm{O}$ emulsions have attracted considerable attention because of their high potential for applications in the field of food science [85], cosmetics [86], and pharmaceutics [87].
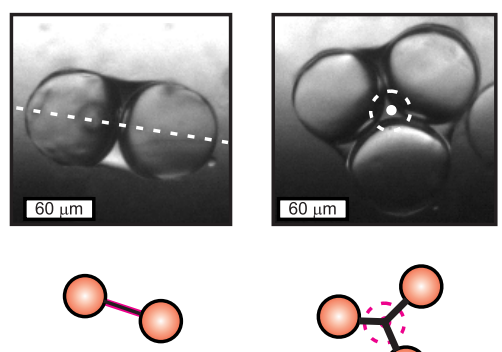

Linear

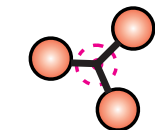

Trigonal

Planar
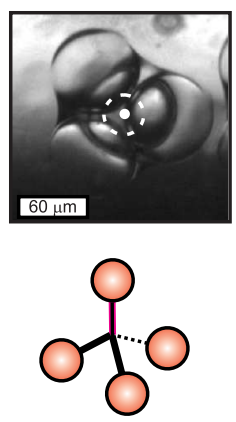

Tetrahedral
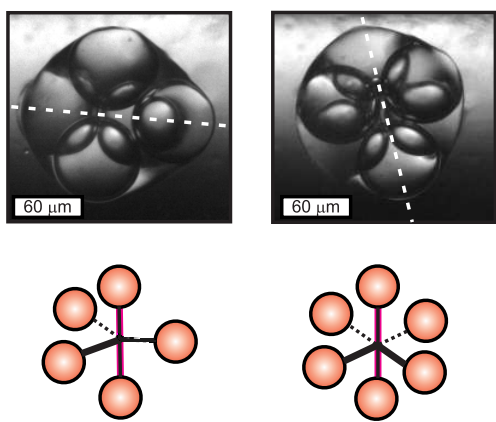

Trigonal Bipyrimid

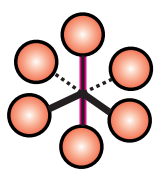

Octahedral

Figure 1.3: Optical micrographs of a double emulsions. If the outer phase volume fraction is extremely low, the inner droplets are compressed together, showing an internal order resembling common molecular arrangements.

\subsubsection{Emulsion stability}

Emulsions (macro- and mini-emulsions) may degrade via a number of different mechanisms including creaming, aggregation, coalescence and Ostwald ripening. Sedimentation, or creaming, results from a density difference between the dispersed and continuous phases, which in turn, will lead the separation of two layers, one of the layers will contain an enhanced concentration of dispersed phase, which may promote aggregation. Aggregation is when two or more dispersed species clump together, possibly touching at some points, and with virtually no change in total surface area.

Coalescence is when two or more droplets fuse together to form a single larger droplet, reducing the total surface area. The rate of coalescence of the droplets in a macroemulsion is stated to be the only quantitative measure of its stability. It can be measured by counting the number of droplets per unit volume of the emulsion as a function of time. The rate at which the droplets of a macroemulsion coalesce to form larger droplets and eventually break the emulsion has been found to depend on a number of factors: (1) the physical nature of the interfacial film (surfactant), (2) the existence of an electrical or steric barrier on the droplets, (3) the viscosity of the continuous phase, (4) the size of the droplets, (5) the two phase volume ratio, and (6) the temperature. 
Ostwald ripening is the expression given to the process whereby the droplet size distribution in an emulsion progressively shifts towards larger sizes. The origin of the effect is the Laplace pressure inside the droplet, cf. Eq. 1.1. As a result of the Laplace pressure, molecules of the dispersed phase diffuse from the high-pressure regions to the low-pressure ones, that is, the small droplets shrink and the larger ones grow as the material is transferred by diffusion through the continuous phase $[68,88,89]$. It might be possible to stabilize emulsions against Ostwald ripening by Osmotic pressure. For that, a small amounts of a third component is added which preferentially dissolves in the dispersed phase and not in the continuous phase. For example the addition of a salt to the water phase can slow the Ostwald ripening of a W/O emulsion.

\subsection{Foams}

When the continuous phase is reduced to a few percent, the topology of an emulsion will be similar to the topology of a foam. Hence, in this section some general introduction to foam system will be outlined. There are some similarities between emulsions and foams. They both consist of a dispersion of a fluid in an immiscible continuous phase. Second, they have a non-zero interfacial tension, $\gamma$, and a marked increase in interfacial area during the emulsification or foaming. Third, the minimum work involved is the interfacial tension multiplied by the interfacial area. Fourth, the system will spontaneously revert to two bulk phases unless there is a surfactant present that prevents coalescence usually by lowering the surface tension, increasing steric interaction, or electrostatic repulsion.

There are two significant differences between emulsions and foams: (1) The surfactants in the interfacial film of a foam cannot dissolve in the dispersed (gas) phase, while in an emulsion the solubility of the surfactants in the liquid being dispersed is a major factor determining the stability of the emulsion. (2) In emulsions, both oil and water can serve as the continuous phase, while in foams, only the liquid acts as the continuous phase.

\subsubsection{Laws of Plateau}

The first detailed description of foam structure was provided by Belgian scientist Joseph Plateau in the 19th century (Plateau, 1873). From an experimental study, Plateau developed a set of rules which govern the equilibrium of foams:

(i) Three soap films meet at angles of $120^{\circ}$. 
(ii) The films form a curved triangular channel known as a Plateau border, cf. Fig. 1.4 .

(iii) Four Plateau borders meet at angles of $109.6^{\circ}$ to form a vertex.

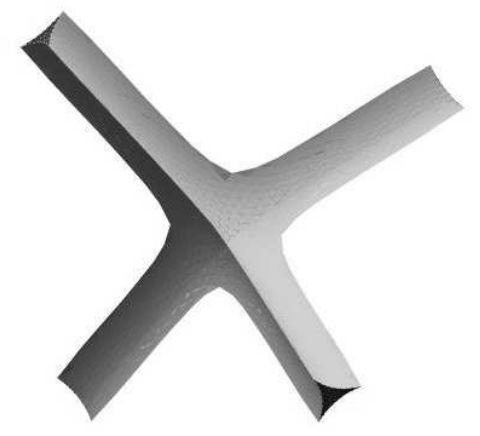

Figure 1.4: The elementary Plateau border junction in three dimensions. Image taken from Ref. [90].

If more than three films or more than four lines meet, the configuration is unstable. One example of topological transition in foam is the T1 transition [90-93]. From a mechanical point of view, the $\mathrm{T} 1$ process corresponds to a transition from one metastable configuration to another, cf. Fig. 1.5 after passing through an unstable configuration where four films meet at one junction (actually, for a small but finite liquid fraction, the instability arises slightly before the four-fold vertex is formed). The spontaneous evolution from one four-fold junction to two three-fold junctions, which involves creation of a new film, is driven by minimization of the surface area.

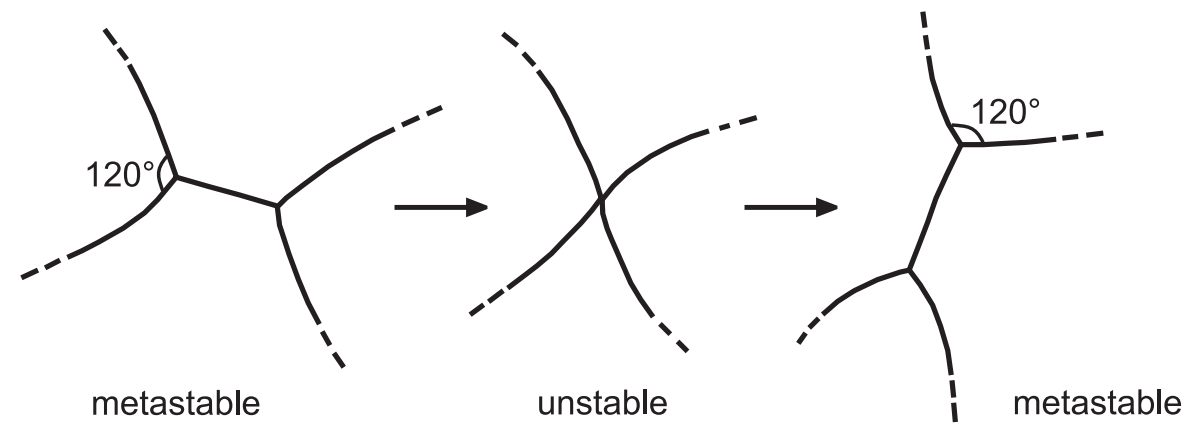

Figure 1.5: A schematic of the $\mathrm{T} 1$ transition. In the $\mathrm{T} 1$ process, a four-fold vertex dissociates into two stable three-fold vertices. Image taken from Ref. [93]. 


\subsubsection{Structure of foams}

During his research Lord Kelvin proposed the following problem: What is the most economical way of partioning space with equal volume of cells? Kelvin's solution, the tetrakaidekahedron, cf. Fig. 1.6 (left) remained the best solution to this problem until 1993 when Denis Weaire and Robert Phelan found a structure with $0.3 \%$ less surface area than Kelvin's structure, which is quite a large difference in this context [90]. The Weaire-Phelan structure uses two kinds of cells of equal volume; an irregular pentagonal dodecahedron and a tetrakaidecahedron with 2 hexagons and 12 pentagons, again with slightly curved faces, cf. Fig. 1.6 (right). To date it has not been proven that the Weaire-Phelan structure is optimal, but it is generally believed to be likely.
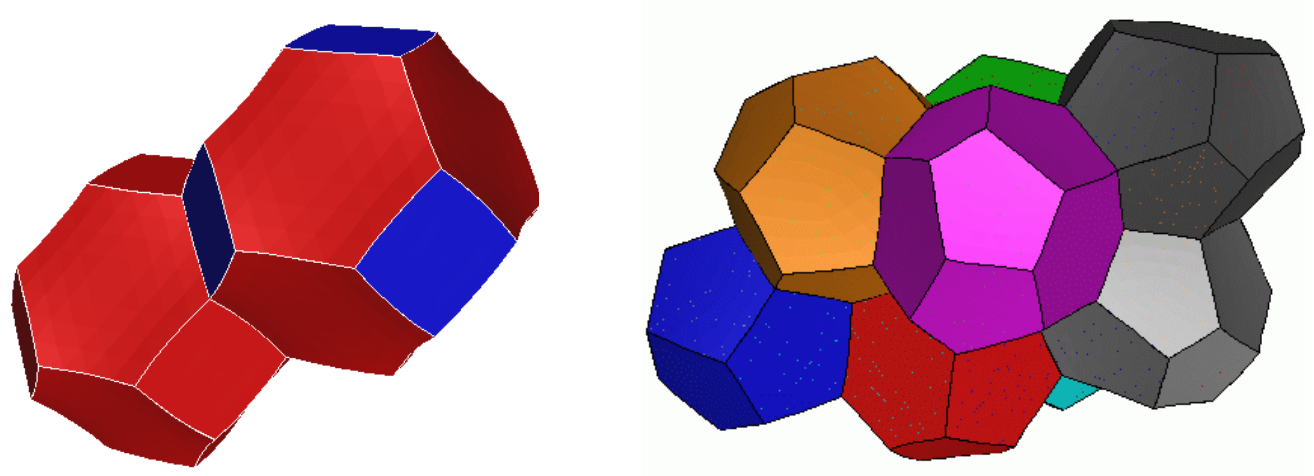

Figure 1.6: Space filling foam structures. Left: Kelvin's tetrakaidecahedron Right: Weaire and Phelan's irregular pentagonal dodecahedron. Images are taken from Ref. [94]. 


\section{Chapter 2}

\section{Materials, Methods and Experimental Techniques}

The generation of emulsions with special properties like droplet size, dispersed volume fraction and production rate is essential for droplet based microfluidics. In order to manipulate emulsion structures in a controlled manner, monodisperse emulsions should be produced insitu with variable volume fraction and droplet size. Thus, emulsion systems, experimental set-up and the various droplet generation techniques used in framework of this thesis were presented in this chapter. A techniques how the microchannels were fabricated is also included. In addition, targeted electrocoalescence of droplets will be described.

\subsection{Emulsion systems}

In general, oil-in-water $(\mathrm{O} / \mathrm{W})$ emulsions are more stable with greater choice of surfactant. However, water-in-oil (W/O) emulsions are good candidates for applications where aqueous phase chemical reactions and bioassays are required. Therefore, $\mathrm{W} / \mathrm{O}$ emulsions are the focus of this thesis. We used three types of emulsion systems in our microfluidic devices, depending on the type of manipulation and the aim.

For passive manipulation, based on "fixed" channel geometries, milipore water (18 M $\Omega . c m)$ and low viscosity organic liquid (Isopar ${ }^{T M} \mathrm{M}$, Exxon Mobil), $\eta_{c}=2.1 \mathrm{mPa} \cdot \mathrm{s}$ were used to make water-in-oil emulsions in microfluidic devices. Non-ionic surfactant, Span $80\left(\mathrm{C}_{24} \mathrm{H}_{44} \mathrm{O}_{6}\right)$, which has a HLB value (see section 1.2.1) of 4 was added to the continuous phase to stabilize the single droplets from coalescing. Span 80 or Sorbitan monooleate is a Sorbitan Ester widely 
used in food products and oral pharmaceuticals. Fig. 2.1 shows the molecular structure of the amphiphilic Span 80 where the hydrophilic sorbitan group acts as the 'polar head' and the hydrophobic oleic acid group acts as the 'nonpolar tail'. The surfactant concentration used in the experiments was $2 \mathrm{wt}$.\%, unless otherwise stated. This emulsion is very stable allowing for long observation times in the microchannels. The interfacial tension, $\gamma$, of our system, which is obtained by the pendant drop technique, is $4.8 \mathrm{mN} / \mathrm{m}$.

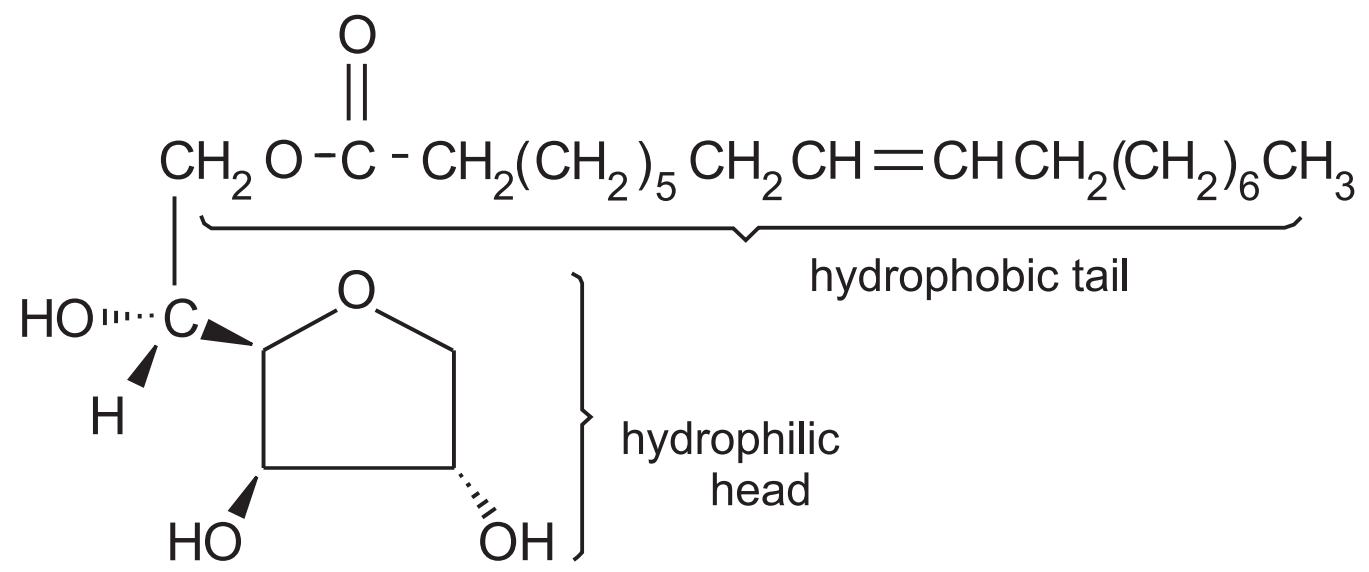

Figure 2.1: Molecular structure of Span 80, showing the hydrophilic head (sorbitan) and hydrophobic tail (oleic acid) parts.

For active manipulation experiments, an oil-based ferrofluid (APG 311, Ferrotec Corporation) was used as the continuous phase. As above, the dispersed phase is aqueous. A ferrofluid is a stable colloidal suspension of sub-domain magnetic particles in a carrier liquid. The carrier liquid can be water, or a variety of oil bases (e.g. organic solvent). The magnetic particles, which have an average size of about $10 \mathrm{~nm}$, are coated with a surfactant which prevents particle aggregation even when a strong magnetic field gradient is applied to the ferrofluid (cf. Fig. 2.2). The surfactant must be matched to the carrier type and must overcome the attractive van der Waals and magnetic forces between the particles [95]. In a gradient field the whole fluid responds as a homogeneous magnetic liquid which moves to the region of highest field strength. This means that ferrofluids can be precisely positioned and controlled by an inhomogeneous external magnetic field. The magnetite concentration in APG 311 ferrofluid was $1.8 \mathrm{wt} . \%$. No further details of the hydrocarbon or the surfactant could be disclosed by the manufacturer; however, we observed excellent emulsion stability without the addition of surfactant to the ferrofluid. The ferrofluid viscosity, density and surface tension are $70 \mathrm{mPa} \cdot \mathrm{s}$ $\left(27^{\circ} \mathrm{C}\right), 940 \mathrm{~kg} \cdot \mathrm{m}^{-3}$, and $30 \mathrm{mN} \cdot \mathrm{m}^{-1}$, respectively. The saturation magnetization is $11 \mathrm{mT}$. 


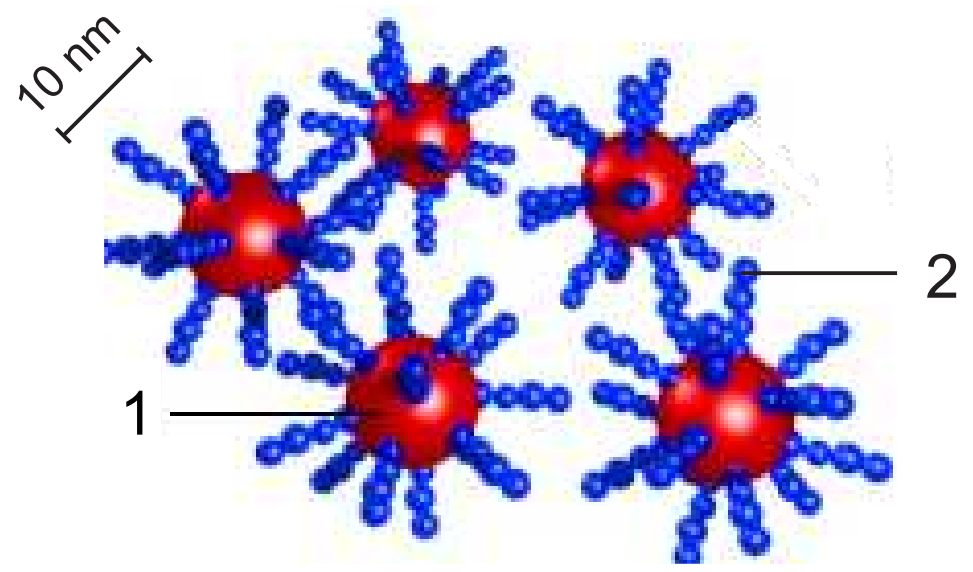

Figure 2.2: An image of magnetic particles in the ferrofluid. 1-magnetic particle which has 10 nm diameter, 2-surfactant.

To investigate fibrin network evolution, a simplified system, consisting of aqueous solution containing the proteins fibrinogen and thrombin (Enzyme Research Laboratory; MobiTec), respectively, were used as dispersed phases. Prior to the experiments the proteins were diluted in appropriate buffers. The continuous phase was Isopar M with 2 wt.\% Span 80.

\subsection{Microchannel fabrication}

When creating droplet microfluidic systems, one of the most critical matters to address is the material used for microchannel fabrication. A large number of microfluidic devices are fabricated using polydimethylsiloxane (PDMS), which is an inexpensive elastomeric polymer. The fabrication of microchannels in PDMS is simple and fast, making it the material of choice for many studies. However, since PDMS undergoes swelling and deformation in the presence of strong organic solvents, other materials with greater solvent resistance, e.g. glass [83] and silicon [96], have also been used. In this thesis, poly(methylmethacrylate) (PMMA) or SU8 photoresist were used to fabricate the microdevices. In the following subsection, two methods for fabricating microfluidic channels in solvent resistance materials are presented. Depending on the dimension of the channels, the devices were fabricated using two different methods: For the larger channel dimensions where the smallest channel width is larger than $200 \mu \mathrm{m}$, the channels could be directly micro-machined in a PMMA block and were closed by a PMMA cover plate. For channel dimensions less than $200 \mu \mathrm{m}$, we developed a photolithographic 
method and bonding technique to form channels in SU8 supported by glass plates.

\subsubsection{Micromachined channels}

Device designs were drawn using CAD-software (Solid Edge), fabricated by micro-machining in a PMMA block (Lutze $\mathrm{GmbH}$ ), and sealed with a $2 \mathrm{~mm}$ thick PMMA cover plate using metal screws, cf. Fig. 2.3. The limitations of the micro-machining technique are determined by mechanical and technical restrictions: Channel width $\geq 200 \mu \mathrm{m}$, channel depth $\geq 10 \mu \mathrm{m}$. PMMA is resistant to the chosen liquids (Isopar M, surfactant, Ferrofluid), is transparent and easily machined (in 3D), making it well suited to our study. An example of a PMMA device is shown in Fig. 2.3. Teflon tubing with an inner diameter of $0.8 \mathrm{~mm}$ was introduced through the big screw (the red screw in the Fig. 2.3) and then prepared with a special flange tool. Then the tube can be pushed onto an orifice using a clamping ring and finally fitted into the thread of the PMMA element. This type of connection can be used for high pressure applications due to the perfect transition between tube and microchannel. If dirt is accumulated in the channel, the device could be opened and washed with ethanol and dried under nitrogen flow.

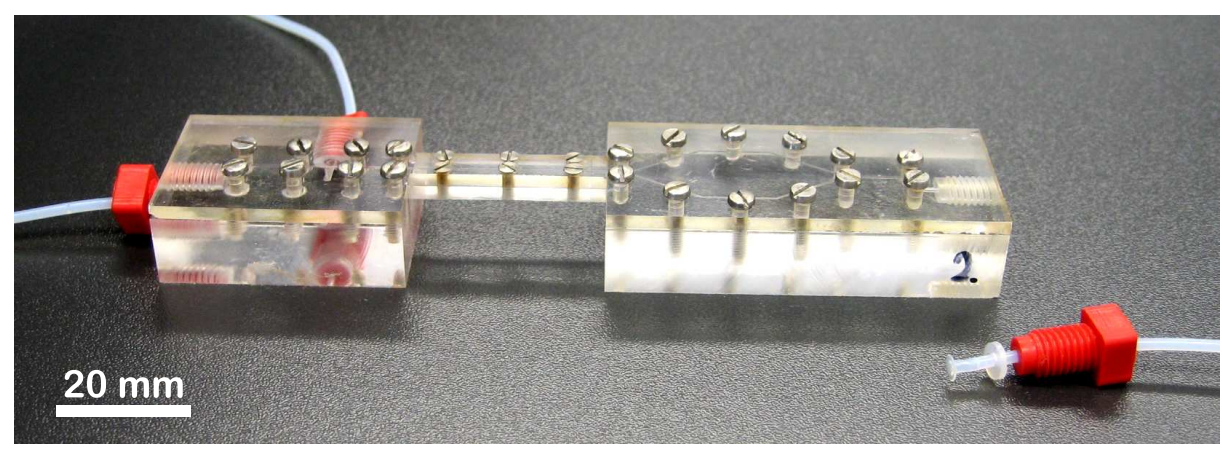

Figure 2.3: Micro-machined PMMA module connected with tubing. The PMMA element is cut from the side in order to bring the pole shoe of the electromagnet close to the channel (for more detail see Chapter 4).

\subsubsection{Photolithography channels}

In order to scale down our devices (for smaller drop volumes), a photolithography of SU8 photoresist was employed [97]. Using this method, microchannels could be fabricated directly in SU8 photoresist with features sizes down to about $10 \mu \mathrm{m}$ [45]. SU8 photoresist is an epoxy 
based polymer designed for MEMS applications, where a thick chemically and thermally stable film is desired. SU8 is a negative photoresist, which means that the exposed portions of the films are crosslinked rendering them insoluble to the developer solution (mr-Dev600). All the chemicals used were bought from Micro Resist Technology GmbH, Germany.

(a)

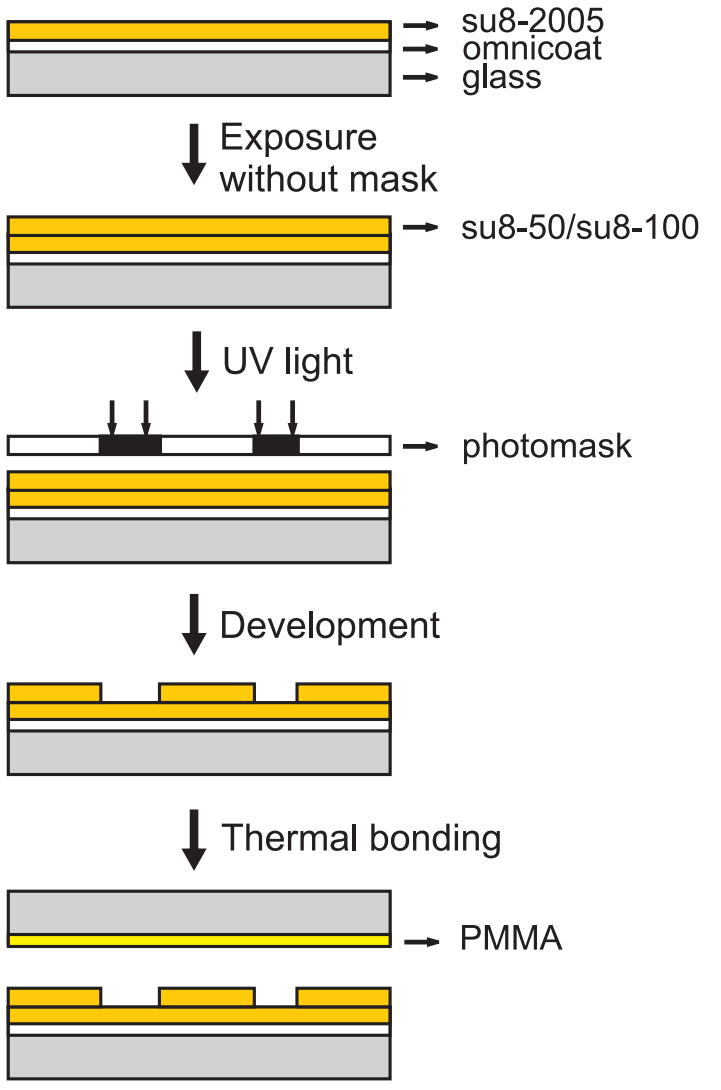

(b)

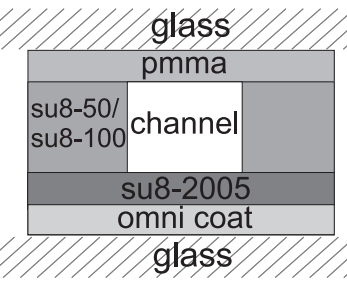

(c)

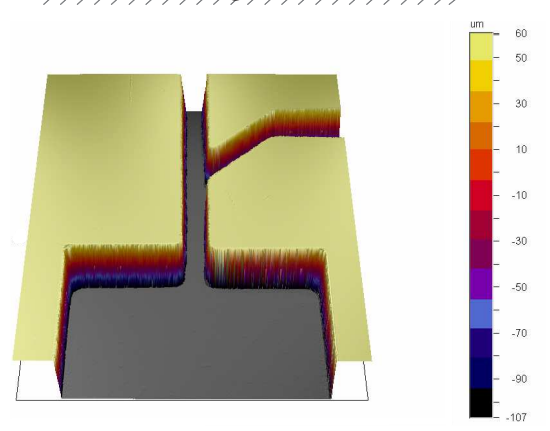

(d)

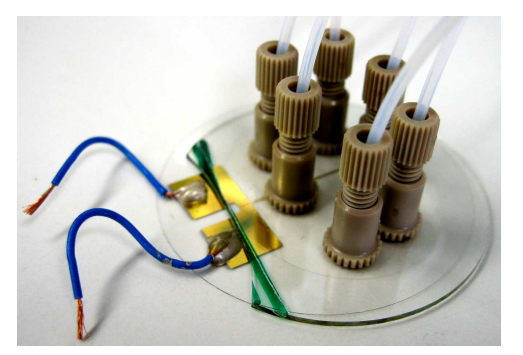

Figure 2.4: Microfluidic device fabrication by photolithography. a) Sketch showing the fabrication steps. The layer information of the microchannel is shown in (b). c) Channel cross section captured by white light interferometry. d) Image of a completed device. The diameter of the glass disks is $50 \mathrm{~mm}$; the channel width is typically $20-100 \mu \mathrm{m}$. The teflon tubing are connected to the device via Nanoports ${ }^{T M}$.

As for the PMMA devices, the device fabrication starts with creating a design in a CAD program. A high resolution commercial image setter then prints this design on a transparency (JD Photo tools, UK). This transparency serves as the photomask in contact photolithography to produce a negative relief in photoresist on a glass substrate. A round glass disk with $50 \mathrm{~mm}$ diameter (VWR), precoated by Omnicoat for better adhesion of SU8 to the glass. Then a 5 $\mu \mathrm{m}$ thick underlayer of SU8-2005 was applied to ensure homogeneous wetting behavior on all 
channel walls, and exposed to UV without mask to crosslink the underlayer. The crosslinked layer was treated with oxygen plasma (PDC-002, Harrick Sci. Corp., USA) for 30 s to render the surface hydrophilic for improved spin-coating of the following coating. Afterwards, SU8100 negative photoresist for the 80-120 $\mu$ m deep channels, or SU8-50 for the 20-50 $\mu$ m deep channels were spun onto the wafer. Spin coating parameters were optimized to achieve the desired film thickness, e.g. to prepare $120 \mu \mathrm{m}$ deep channels, $1500 \mathrm{rpm}$ for $30 \mathrm{~s}$ with $5 \mathrm{~s}$ ramping time was used. Subsequently, the sample was soft baked in order to evaporate the solvent and densify the film. Soft baking times were based on the film thickness. For $120 \mu \mathrm{m}$ layer thickness, the samples were baked for 13 mins at $65^{\circ} \mathrm{C}$ and $37 \mathrm{mins}$ at $95^{\circ} \mathrm{C}$. Then, the samples were exposed to UV light $(\lambda=364 \mathrm{~nm}$ ) in the maskaligner (Karl Suss MJB 3 UV 300/400) for $30 \mathrm{~s}$ through the photomask. Upon exposure, the cross-linking proceeds in-two-steps; the formation of a strong acid during the exposure process is followed by an acid-initiated, thermally driven epoxy cross-linking step during the post exposure bake. After exposure, the sample is post-baked for a time which depends on the thickness of the photoresist $\left(1 \mathrm{~min}\right.$ at $65^{\circ} \mathrm{C}$ and $7 \mathrm{mins}$ at $95^{\circ} \mathrm{C}$ for $120 \mu \mathrm{m}$ thick resist; $1 \mathrm{~min}$ at $65^{\circ} \mathrm{C}$ and $2 \mathrm{mins}$ at $95^{\circ} \mathrm{C}$ for $30-50 \mu \mathrm{m}$ thick resist). The samples were rinsed with developer to remove the non-crosslinked regions, which were shaded by the mask. For high aspect ratio channels a few seconds of ultrasonication in the developer solution is needed to fully develop the channels. Inlets were drilled into the supporting glass substrate through the inlets of structured photoresist. The channels were closed by thermally bonding a second glass disk coated with a $10 \mu \mathrm{m}$ thick PMMA layer at $160^{\circ} \mathrm{C}$ using a mechanical press. To perform electrocoalescence in the microfluidic device, prior to bonding, gold electrodes with an underlying chromium adhesion layer were thermally vapor deposited at $10^{-6}$ mbar onto the cover glass. Wires were glued to the electrodes using electrically conducting epoxy resin (ITW Chemtronics ${ }^{\circledR}$ ) to contact the evaporated electrodes prior to PMMA layer. Teflon tubing was connected to the microdevice via Nanoports ${ }^{T M}$ (UpChurch), which were bonded to the microchip according to the company protocol (see Fig. 2.4). The tubing was connected to Hamilton syringes that were driven using home-made syringe pumps controlled by a program written in LabView (National Instruments Corporation). The recipe for the photolithography microdevice fabrication can be found from appendices, pp. 99. 


\subsection{Experimental set-up}

A Leica Z16 APO optical microscope with a Leica L5 FL light source cf. Fig. 2.5 a, was typically used to image the emulsion. Images and movies were recorded using a high resolution CCD camera (PCO 1600) (and CamWare software). When needed, a high speed CMOS camera (PCO $1200 \mathrm{hs}$ ) which is able to capture more than 600 frames per second was used. Typical exposure times ranged from $500 \mu$ s to $50 \mathrm{~ms}$, depending on the flow velocity. The flow rates were adjusted using custom-made syringe pumps controlled by programs written in LabView (National Instruments Corporation), cf. Fig. 2.5 b. Using glass syringes (Hamilton) with various diameter, flow rates between $10 \mu \mathrm{l} / \mathrm{h}$ and $1 \mathrm{ml} / \mathrm{h}$ could be achieved.

To visualize the fibrin network we used a high resolution optical microscope, Zeiss Axiovert 135 with a 60x 1.25 NA oil objective and an ebx75 Xenon illumination source equipped with the appropriate filters to perform fluorescence experiments using an excitation wavelength of around $546 \mathrm{~nm}$. For the fluorescence microscopy images were recorded using a very sensitive camera PCO sensicamQE camera (and CamWare software). The typical exposure times used to image fibrin network were from $700 \mu$ s up to $10 \mathrm{~ms}$. Further analysis was conducted using 'Image-Pro Plus 5.0' and ImageJ image processing software.

In order to manipulate water-in-ferrofluid emulsions, we used permanent magnets made of Neodymium-Iron-Boron (NdFeB) (IBS Magnet, Ing. K.-H. Schroeter, Germany) or an electromagnet (Model 3470, GMW Associates, San Carlos, California, USA) capable of producing a magnetic field up to $1.8 \mathrm{mT}$ as is explained in detail in the chapter 4 .

a)

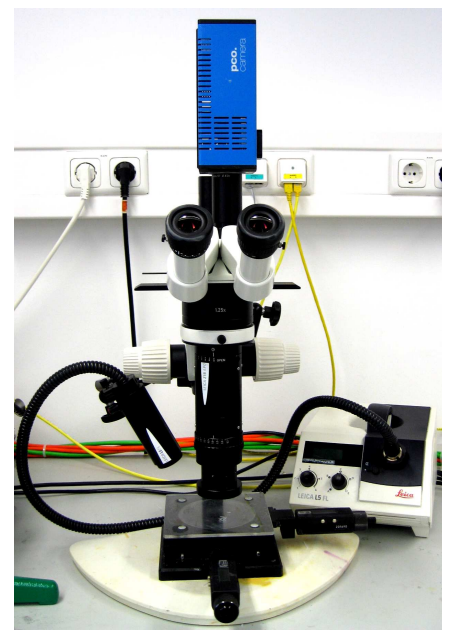

b)

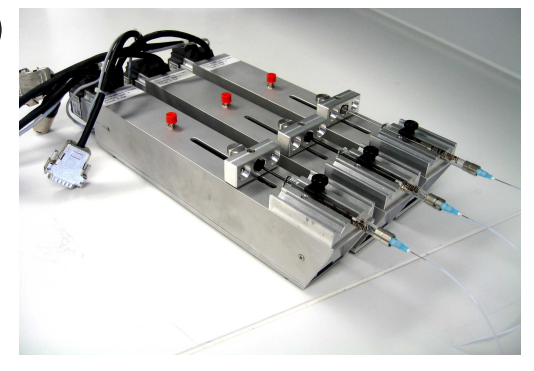

Figure 2.5: a) Z16 APO Leica microscope with a Leica L5 FL light source b) syringe pumps. 


\subsection{Monodisperse emulsion production in microchannels}

The power of droplet-based microfluidic systems lies in the formation of uniform droplets, thus fine control over the size and monodispersity of droplets is of the utmost importance for the further microfluidic processing. Although the same basic principles and materials are used, a variety of techniques have been developed for droplet generation based on the geo-metry of microchannel. Controlled formation of droplets in a microfluidic system has been developed in a various different ways including T-junction shear, [40, 41] flow focusing [42, 43] and step emulsification [44, 45]. T-junction emulsification shears the dispersed phase in the perpendicular flow of the continuous phase, whilst flow focusing forces the dispersed phase into a thin thread which ruptures via Rayleigh-Plateau instability. An alternative method, namely "step emulsification", offers a generation of monodisperse, high dispersed phase volume fraction emulsions in a microchannel. However, when monodisperse, the droplets exhibit extremely well-defined foam like spatial order which provides an additional handle for microfluidic processing as we will explain later in this chapter.

\subsubsection{T-junction emulsification}

Due to it's simplicity the most popular microfluidic device used for the generation of droplets is a T-junction geometry, first incorporated into a microfluidic chip by Thorsen et al. [40], and subsequently used for formation of droplets [40, 41], and bubbles [98], for formation of double emulsions [82], and in a host of analytical applications [99, 100]. Recently, Whitesides et al. reported the mechanism of droplet break-up in terms of the continuous phase viscosity and channel dimensions [101].

In the T-junction configuration, the inlet channel containing the dispersed phase intersects the main channel which contains the continuous phase cf. Fig. 2.6 a and Fig. 2.6 b. The two liquid phases form an interface at the junction, and as the fluid flow continues, the tip of the dispersed phase enters the main channel. The stream of the dispersed phase penetrates into the main channel and a droplet begins to grow; the pressure gradient and the shear force generated by the continuous phase distort the droplet in the downstream direction until the neck of the dispersed phase thins and eventually breaks the stream into a droplet cf. Fig. 2.6 b. The size of the produced droplets can be varied in a wide range by altering the fluid flow rates, the channel dimensions or by changing the relative viscosity between the two phases. Therefore, the T-junction design is used in our experiments where the drop size needs to be varied in a 

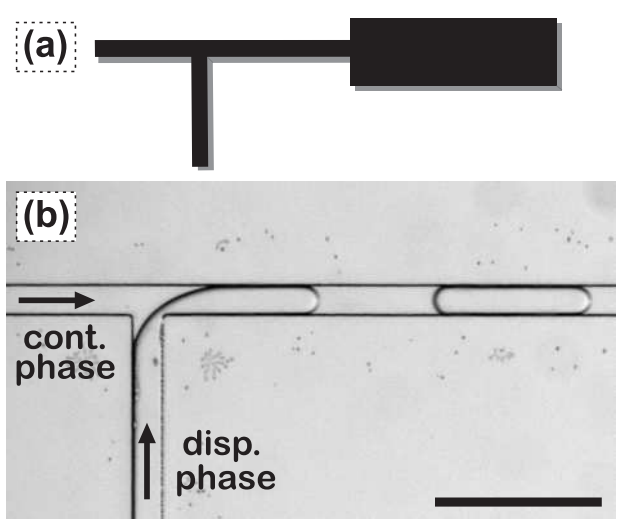
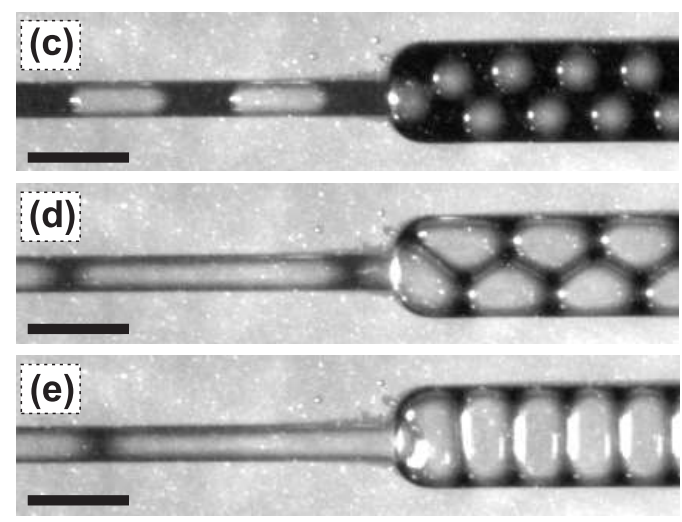

Figure 2.6: a) Schematic of T-junction microdevice. b) Droplet formation at T-junction. After production, the channel width was increased to arrange the droplets. By increasing the drop size to channel width ratio (c-e), different droplet arrangements can be obtained.

wide range. However, the limited range of volume fraction, critical flow velocity needed for droplet break-up, monodispersity is not excellent and it is difficult to change the production frequency without changing the droplet size are the drawbacks of the T-junction method.

\subsubsection{Hydrodynamic flow-focusing}

Flow-focusing geometry is first introduced to generate a bubbles in a real 3D geometry [43] and later used to generate a droplets in a microfluidic device [42]. In the flow-focusing configuration, the dispersed and continuous phases are forced through a narrow region (orifice) in the microfluidic device. The outer fluid exerts pressure and viscous stresses that force the inner fluid into a narrow thread, which then breaks inside or downstream of the orifice cf. Fig. 2.7. The droplet generation in the flow-focusing design can be achieved without surfactants, although their use might support droplet formation. The droplet size is not limited by the injector and orifice size, i.e. droplets can be much smaller than the orifice size and can be adjusted by changing the relative flow velocity of the two fluid phases. Examples of flow focusing applications include the generation of homogeneously sized water droplets in oil [42], multiple emulsions [83, 102], and preparation of polymer microspheres [103, 104]. However, using flow-focusing method one can not achieve large volume fractions of the dispersed phase and a wide range of drop sizes. A device geometry is more complicated because of two streams needed for the continuous phase. 


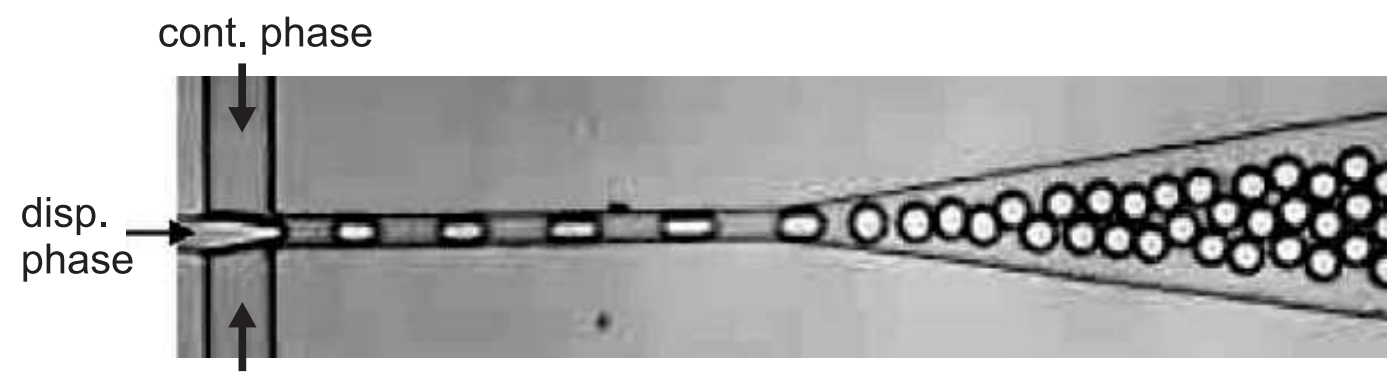

cont. phase

Figure 2.7: An example of droplet production by a hydrodynamic flow focusing design [42].

\subsubsection{Step emulsification}

Emulsions with a high dispersed phase volume fraction can be prepared in a microchannel via multistep splitting of large droplets (with respect to the channel dimension) into a resulting daughter droplets [105]. Multistep splitting requires a branched microchannel network which leads to higher flow resistance and a potentially an increased polydispersity of the droplets associated with each splitting step. Alternatively, step emulsification allows for the generation of monodisperse, high dispersed phase volume fraction emulsions in a microchannel which is well-suited for drop-based microfluidics [44, 45]. In the present subsection we will describe the mechanism of droplet formation at a step in the microchannel. Step emulsification was the emulsification method of choice used in most of the experiments in this thesis. The initial discussion will focus on the main characteristics of a step emulsification device and will continue with two alternative microchannel designs that employ the same drop break-up mechanism.

\subsubsection{Step emulsification device using PMMA channels}

A schematic of the step emulsification device fabricated by micromachining in PMMA is shown in Fig. 2.8 a. The microdevice consists of a T-junction at a shallow rectangular channel connected to a three-dimensional channel. The dispersed phase is injected into the continuous phase where it is guided in a shallow channel and stabilized by the surrounding continuous phase and the channel walls. When the stream enters to the deep channel at the topographic step, the continuous stream of the dispersed phase breaks-up into single drops. For this device, drop formation may proceed via three mechanisms: shear at the junction, jet break-up in the larger channel, or via step emulsification [44]. 
(a)

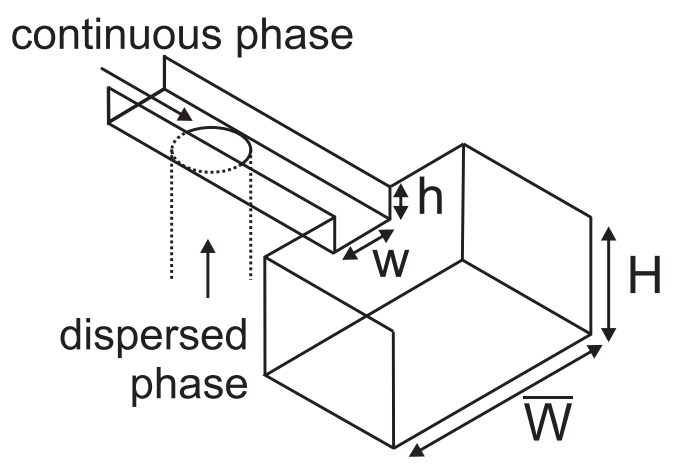

(b)

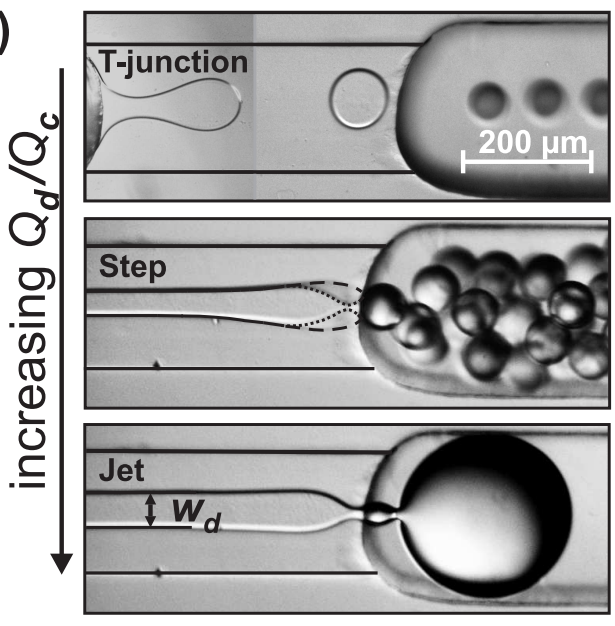

Figure 2.8: a) Schematic of a step-emulsification device fabricated by micromachining in a PMMA. b) Optical micrograph of drop detachment via T-junction, step, and jet break-up mechanisms (top to bottom). Image taken from Ref. [44].

Different flow conditions in a step emulsification device can be achieved by varying the relative flow ratio of the two phases involved. When the flow rate of the dispersed phase, $Q_{d}$, is small compared to the flow rate of the continuous phase, $Q_{c}$, emulsification occurs at the T-junction where the two liquids meet, cf. Fig. $2.8 \mathrm{~b}$, top. At high relative flows, $Q_{d} / Q_{c}$, the two liquids stream through the high aspect ratio channel, and a Rayleigh-Plateau type instability develops downstream from the step, cf. Fig. 2.8 b, bottom. The monodispersity of the produced droplets and the limited volume fractions are the undesired properties of both T-junction and the Jetinstabilities.

Alternatively, step emulsification occurs at intermediate $Q_{d} / Q_{c}$ maintaining excellent monodispersity (coefficient of variance $<1.5 \%$ drop diameter), with adjustable volume fraction (< $96 \%$ dispersed phase) and production frequency (up to several $100 \mathrm{~Hz}$ ) [44]. The main characteristic is that step emulsification is directly induced at the topographic step, where the shallow channel merges into the deeper channel, cf. Fig. 2.8 b, middle. The physical reason for this transition is that the flow profile is effectively two-dimensional in the shallow channel, but is three-dimensional in the deep channel. Since there is no jet instability in two dimensions, the flow is stable up to the step, where it abruptly breaks up.

To display the boundary between the three different droplet break-up mechanism, the width of the dispersed phase stream (definition see Fig. $2.8 \mathrm{~b}$ ) normalized by the height of the channel, $w_{d} / h$ is plotted as a function of the capillary number, $\mathrm{Ca}=\eta v / \gamma$, where $\eta$ is the dispersed 
phase viscosity, $v$ is the velocity of the dispersed phase, and $\gamma$ is the liquid/liquid interfacial tension in the presence of a surfactant.

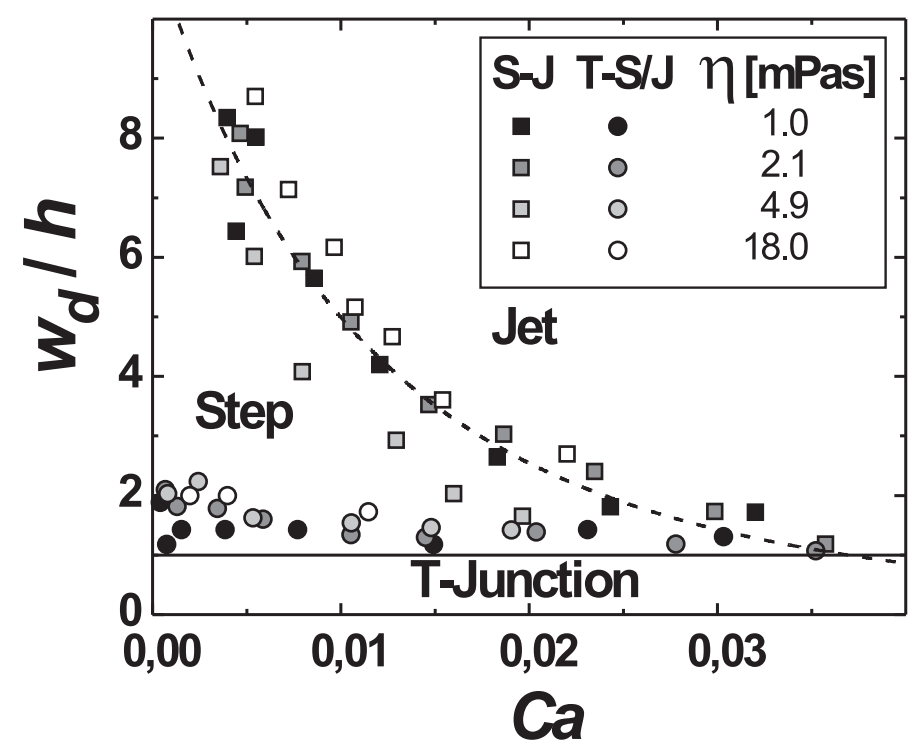

Figure 2.9: Diagram showing the three different mechanism for drop formation: T-junction, step, and jet instabilities. Squares and circles indicate the step-jet and T-step (or jet) boundaries, respectively, for water and three glycerol-water mixtures. The channel height, $h$, was $22 \mu \mathrm{m}$. Data taken from Ref. [44].

Another very useful aspect of step emulsification technique for our study is the produced drop size is insensitive to the total flow rate, provided the relative flow rate $Q_{d} / Q_{c}$ is constant. Therefore, the production rate can be adjusted independent of the drop volume by varying the total flow rate, $Q_{t}$, while keeping the relative flow rate, $Q_{d} / Q_{c}$ fixed. Thus, the frequency of the droplet production can be varied easily from a few to several $100 \mathrm{~Hz}$ while maintaining the drop size constant.

Using step emulsification technique we were able to prepare highly ordered emulsions with a wide range of the dispersed phase volume fraction, cf. Fig. 2.10 by adjusting flow parameters in a single device. This is the main feature of the step emulsification device which provides the possibility to study the organization and manipulation of emulsion arrangements as a function of the dispersed volume fraction as we will discuss later. 

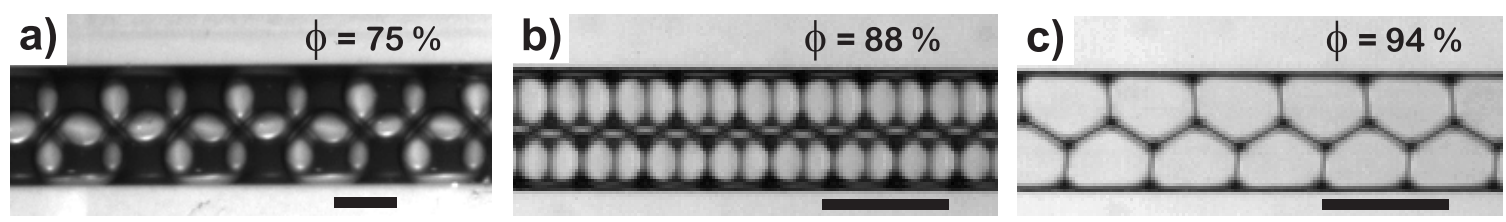

Figure 2.10: A micrograph of emulsion arrangements with various volume fraction produced by a step emulsification device. The continuous phase was a ferrofluid. A four-row arrangements at $\phi=75 \%$ (a) and at $\phi=88 \%$ (b), respectively. c) A zigzag arrangement at $\phi=94 \%$. Scale bar: $250 \mu \mathrm{m}$.

\subsubsection{Step emulsification device using photolithography}

To downscale the channel size compared to the michromachined PMMA device, we optimized the geometry of step emulsification device, essentially rotated by $90^{\circ}$, to enable its fabrication using single step photolithography as described in the methods subsection 2.2.2 however, the generation of droplets still occurs via step emulsification [45]. A schematic of our device is shown in Fig. 2.11 a. As explained in the previous section, the dispersed and continuous phases enter into a high aspect ratio channel $(\mathrm{b} / \mathrm{a} \geq 4)$, which precedes a much larger channel (dimension $\mathrm{b}$ and $\mathrm{c}$ ) of dissimilar aspect ratio $(\mathrm{b} / \mathrm{a} \gg \mathrm{b} / \mathrm{c})$. The dispersed phase

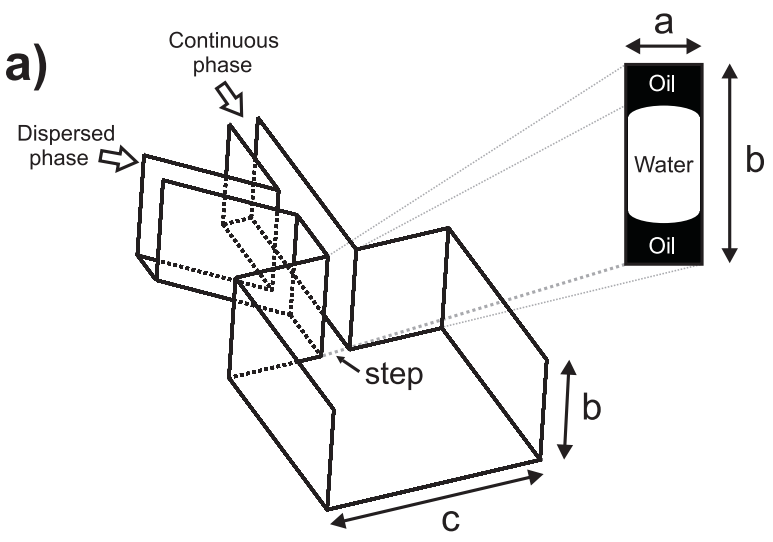

b)

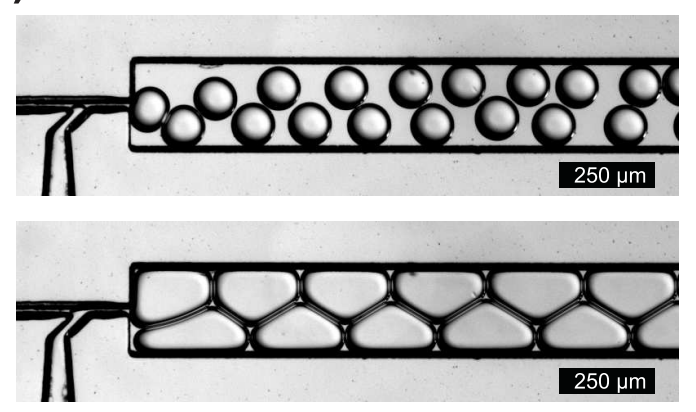

Figure 2.11: a) Schematic of a single step-emulsification device fabricated by photolithography. The dispersed and continuous phases feed into the high aspect ratio channel (b/a $\geq$ 4), which precedes much larger channel (dimension $b$ and $c$ ) of dissimilar aspect ratio (b/a $\gg$ $\mathrm{b} / \mathrm{c}$ ). The typical channel dimensions used in our experiments are: $\mathrm{a}=20 \mu \mathrm{m}, \mathrm{b}=80 \mu \mathrm{m}$ and c $=250 \mu \mathrm{m} \mathrm{b}$ ) Optical micrograph of drop formation via (a) T-junction, (b) Step emulsification mechanisms. 
forms a coflowing stream in the high-aspect ratio channel, the cross-section is illustrated top right in Fig. 2.11 a, where the dispersed stream is stabilized by the side walls of the channel. As there can be no Rayleigh instability in two-dimensions, the stream is stable up to the step, where the flow profile becomes three dimensional and the coflowing stream immediately ruptures. This mechanism minimizes the influence of fluctuations, leading to extremely monodisperse droplets.

\subsubsection{Double step emulsification device}

As it will be discussed in chapter 6, in order to perform chemical reactions, we preferentially need at least two droplet productions which could produce an independent droplets that are containing different chemicals. Therefore, the application of such a single step emulsification device was extended further to produce two different types of monodispersed droplets in a strictly alternating way. However, step emulsification device needs a high aspect ratio (height/width) channels to stabilize the co-flowing stream by channel geometry, which makes it difficult to observe for imaging of fibrin networks in our study. For this reason, we used a microdevice containing double T-junction configurations, where channels could be fabricated as shallow as possible for advantage of network visualization. Although a double step emulsification technique was not used in this thesis, the principle of a device operation will be shortly outlined in the present subsection.

When combining two emulsification units into a single device, as shown in Fig. 2.12 a, we were able to produce two distinct groups of droplets in a fixed number of 1:1 ratio, with each group having identical droplets with excellent monodispersity. The crucial feature is that the two production units synchronize themselves via a pressure cross talk caused by the formation of the droplets. As an example, two different droplet arrangements which are produced by double step emulsification technique are shown, a three row and a zigzag arrangement having a dispersed phase volume fraction of $76 \%$ and $88 \%$, respectively (cf. Fig. 2.12 b). Droplets produced by the second production unit (bottom channel) are painted in order to distinguish them from those produced by first production unit (upper channel).

The principle of double coflowing device e.g. the drop formation mechanism, is the same as the single step emulsification device except there are two production units combined into a single chip and the produced droplets could be sent to one main channel. How do they synchronize themselves is as following; the obstruction of one channel forces liquid to flow 

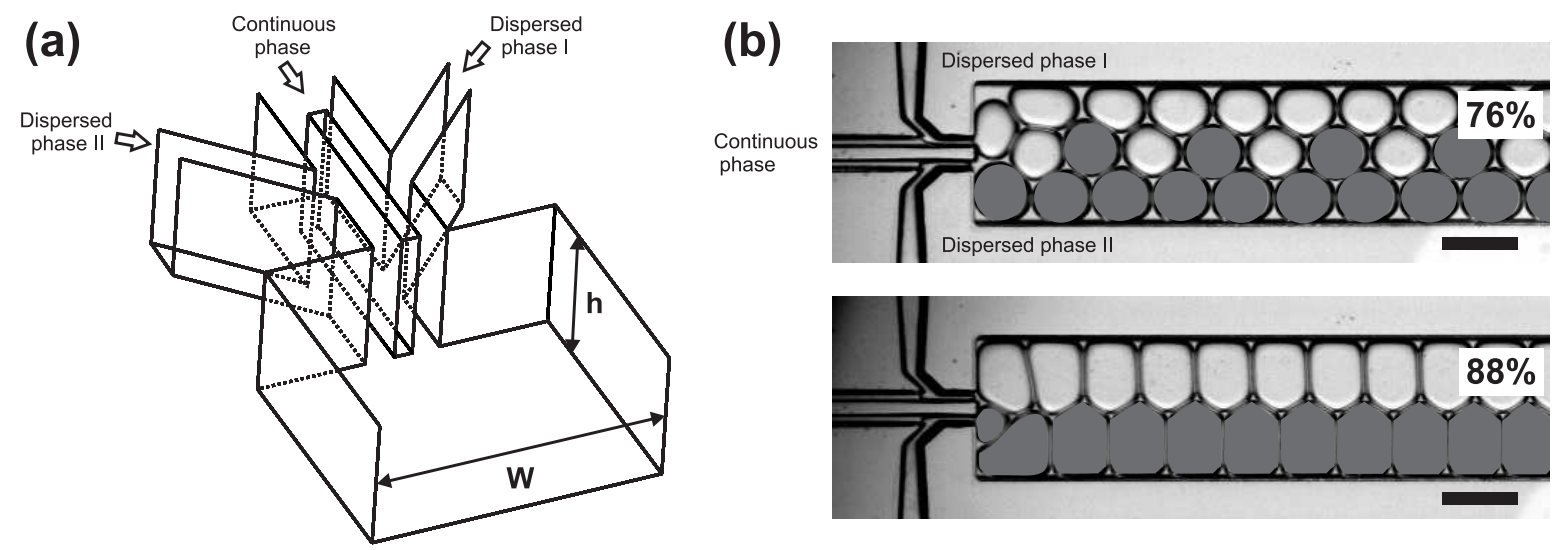

Figure 2.12: a) Schematic of a double step emulsification device with two combined droplet production units. b) Optical micrograph of a three row and a zigzag droplet arrangement produced by double step emulsification device. The dimensions typically used in our experiments: $\mathrm{W}=360 \mu \mathrm{m}, \mathrm{h}=120 \mu \mathrm{m}$, coflowing channel width and length are $35 \mu \mathrm{m}$ and $120 \mu \mathrm{m}$, respectively. The droplets of the dispersed phase II was painted to distinguish them form the droplets of dispersed phase I, scale bar: $250 \mu \mathrm{m}$.
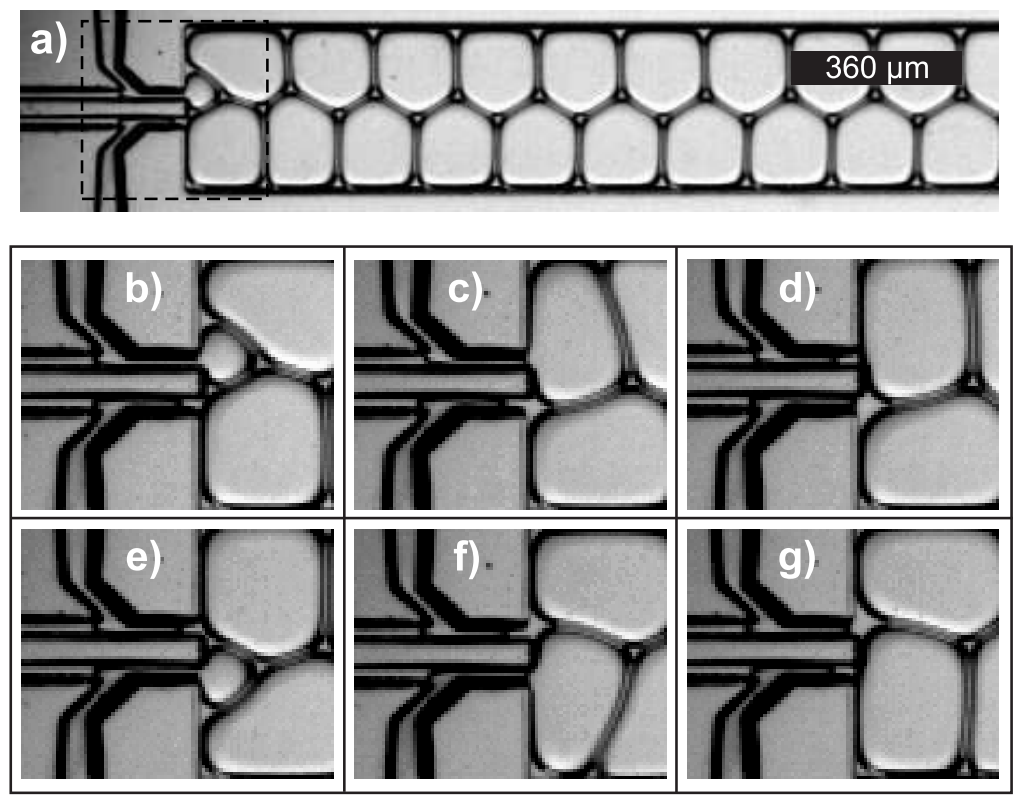

Figure 2.13: Time series of droplet production with double step emulsification device. Dashed lines in the micrograph (a) denote the area in the following micrographs (b)-(g) for better visibility. 
through the other. This stops a droplet forming in the other (second) channel until the droplet is fully formed (upper channel for the dispersed stream in Fig. $2.13 \mathrm{~b}$, c). After the drop is formed from the first channel (upper channel in Fig. $2.13 \mathrm{~d}$ ) the pressure in the second channel (dispersed inlet) is higher than in the first channel, so the next drop must come from the second channel (bottom channel in Fig. 2.13 e) and the cycle is repeated (bottom channel in Fig. 2.13 f, g). The resulting droplet production is strictly alternating.

When the volumetric flow rates are equal for the dispersed phase I and II at the respective production unit $\left(Q_{I}=Q_{I I}\right)$, the corresponding droplet frequency ( $\omega_{I}$ and $\omega_{I I}$ respectively) are identical, the coefficient of variance of the droplet diameters was less than $1.5 \%$ exhibiting excellent monodispersity equivalent with the single step emulsification device [106]. If the volumetric flow rate, $Q_{I}$ at the production unit I is significantly larger than $Q_{I I}$ at the production unit II, two droplets with different volumes are produced with the droplet frequency at the second production unit is higher than the first production unit $\left(\omega_{I}<\omega_{I I}\right)$. The precise alternating droplet production and the excellent monodispersity makes the double coflowing device ideal for many application where chemical reactions are needed.

\subsubsection{Manipulating the volume fraction of the emulsion after production}

The dispersed phase volume fraction is a control parameter, which determines the arrangement of droplets of a certain size in a channel with fixed dimensions as we will discuss later in the last chapter of this thesis. It is therefore needed to adjust the dispersed volume fraction of the emulsion when manipulating the emulsion arrangements. For both T-junction and step emulsification techniques, we can tune the volume fraction of the emulsion by changing the flow rate of the liquid phases involved. However, typically the droplet size is varied simultaneously when adjusting the volume fraction by the flow parameter of one phase. To vary the dispersed volume fraction continuously while keeping all the droplet production parameter constant (in particular without waiting time until the new flow conditions are equilibrated) we thus introduced a side channel to selectively infuse or withdraw the continuous phase. The emulsion "dryness" is then adjusted without affecting the drop size. The lateral dimension of the side channel should be much smaller than the drop size in order to avoid the droplets escaping through the side channel, cf. Fig. 2.14. Provided the droplets size is large enough compared to the side channel width they do not escape through the narrow channel due to the increase in interfacial area associated with droplet deformation. The interfacial tension of the water/oil interface tends to minimize the surface area of the droplet for a given channel 
geometry, therefore the droplet arrangement is determined by the microchannel geometry and the droplet size, however the dispersed phase volume fraction determines the packing density. By controlling the volume fraction of the dispersed phase via side channel it is possible to find many different meta-stable droplet arrangements, as will be discussed in the following chapters of this thesis.

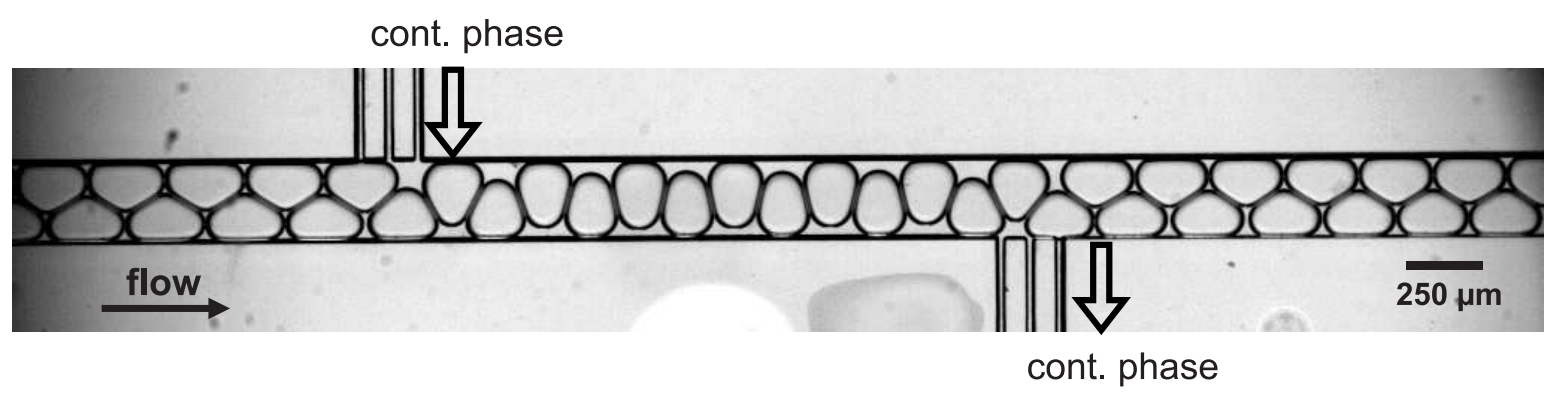

Figure 2.14: The emulsion structures can be altered according to volume fraction. The lateral dimension of side channel is $30 \mu \mathrm{m}$. The flow direction is from left to right as indicated by the solid arrow. The open arrows at the side channel indicate the flow direction of the continuous phase.

\subsection{Droplet coalescence}

Another fundamental aspect of droplet-based microfluidics is coalescence which may be achieved by colliding oppositely charged droplets [38], or forcing droplet pairs through a channel restriction [51, 107, 108] as shown in Fig. 2.15. Droplets A and B are produced at two production units and merged as they pass through the geometrical constriction. When pairs of droplets reach the constriction, the velocity of the first droplet is reduced and the second droplet is pushed towards the first one which destabilizes the oily lamella between the droplets. This process results in the formation of a coalesced droplet C. Surface modification can also be used to induce fusion of droplets [109]. It does not require active elements nor synchronization of droplets and any number of droplets can be fused by a single step. Rows of pillars structured within the microfluidic channel network could also initiate the passive merging of droplets [52]. However, these methods are not easily transferable to gel emulsions due to the dense packing of droplets. Instead we induce coalescence in gel emulsions using electrocoalescence $[53,54]$, where the droplets experience an external electric field. In this 
case the coalescence can be induced locally, and consequently, specific droplet pairs may be targeted for coalescence [36].

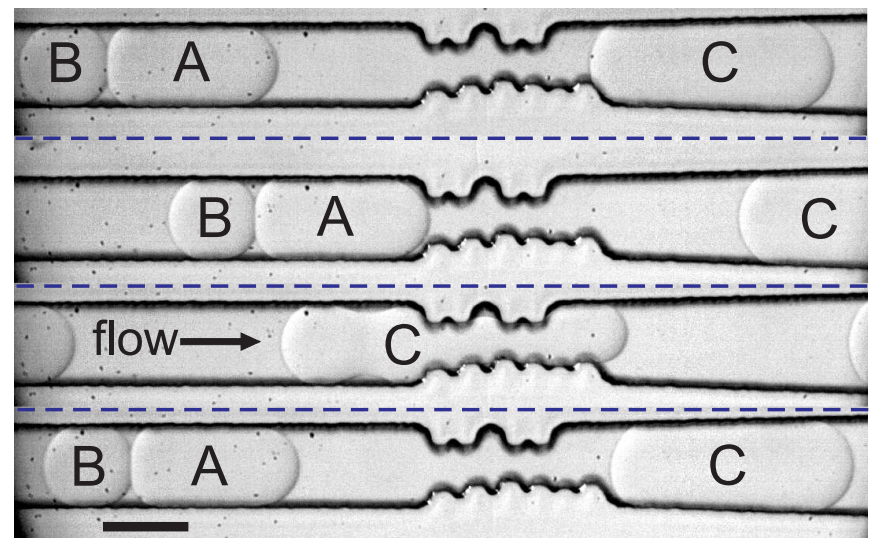

Figure 2.15: Time series of snapshots of droplets merging via a geometrical constriction. Droplet pairs A and B merge as they pass through the constriction, resulting in a coalesced droplet C. Scale bar: $160 \mu \mathrm{m}$. Image taken from Ref. [107].

The first approach of electrocoalescence was to subject pairs of droplets to a high electric field $[38,54]$. The high voltage method is not convenient especially for sensitive biological species that might be encapsulated in the droplets in particular since the droplets are exposed for a fairly long time to the large potential difference. Alternatively, low potential electrocoalescence, which is used in this thesis has been demonstrated by Priest et.al. [36]. The mechanism of electrocoalescence has been explained as an electric field induced dynamic instability of the thin oil lamella separating two droplets [110]. An example for electrocoalescence is illustrated in Fig. 2.16 for bamboo and zigzag droplet arrangements travelling along a microchannel. Applying a short pulse $(100 \mathrm{~ms})$ of $1 \mathrm{~V}$ d.c. between the electrodes induces local coalescence of droplet pairs as they pass the electrodes. By optimizing the electrode dimensions and geometry, targeted coalescence of specific droplet pairs is possible in foam-like arrangements. For instance, for the bamboo arrangement in Fig. 2.16a, the pulse frequency was adjusted to target every third lamella, so that the larger coalesced droplets were separated downstream by a single uncoalesced droplet. For the zigzag arrangement, electrocoalescence was induced perpendicular to the channel using thin electrodes (not wider than the droplet diameter), which were slightly off-set, commensurate with the droplet arrangement.

The required electrical potential is proportional to the lamella thickness, $U \propto d$ and it was found to be independent of the aqueous phase conductivities, cf. Fig. $2.16 \mathrm{c}$. The applied 
a)

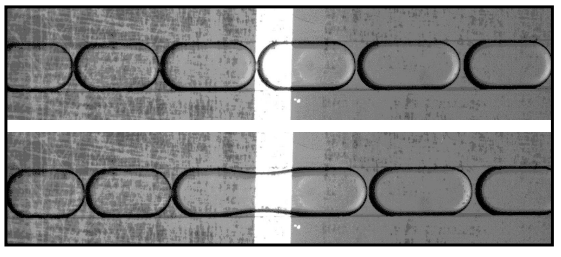

b)

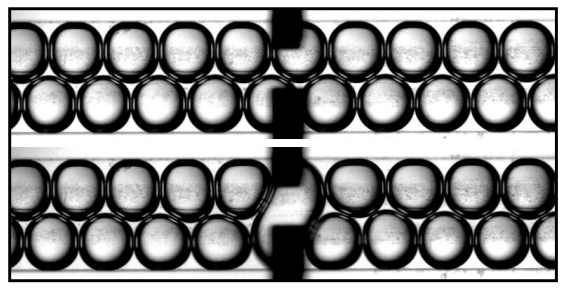

c)

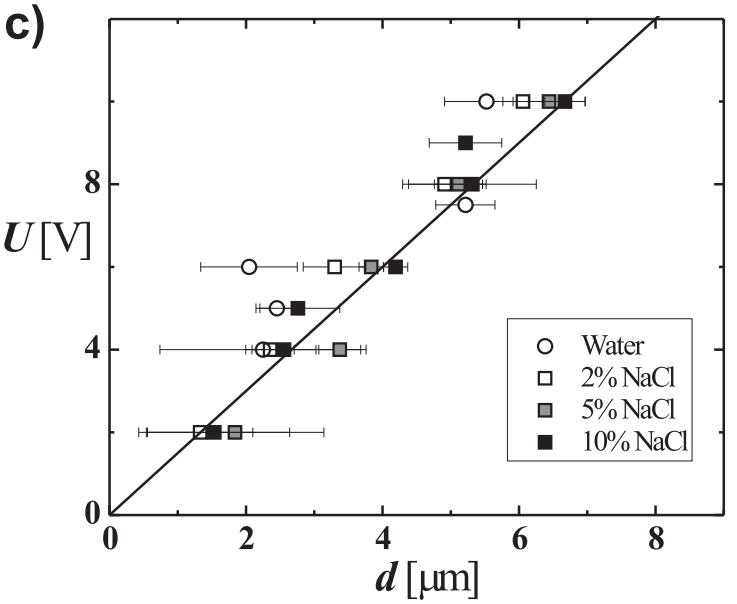

Figure 2.16: Targeted electrocoalescence of droplets within (a) a bamboo arrangement and (b) a zigzag arrangement. The bright line in (a) perpendicular to the microchannel is the gap between the gold electrodes. In (b), the electrodes are indicated by the black rectangles. (c) Applied potential as a function of the lamella thickness for a various salt concentration. Images taken from Ref. [36].

potential for electrocoalescence must be sufficient to rupture the lamella. In contrast, applying an excessive potential difference can lead to indiscriminate coalescence along the length of the channel.

To explain the underline process of the electrocoalescence work presented here, one might consider the possibility of a field-induced dynamic instability of the oil/water interface. In fact, it is well known that when a liquid dielectric film between conducting media is subject to a potential difference, the capillary waves of its bounding interfaces become unstable in a whole range of wave numbers, $q \in\left[0, q_{0}\right]$, where $q_{0}$ depends on the applied voltage. One obtains [110]

$$
q_{0}=\sqrt{\frac{\epsilon \epsilon_{0}}{\gamma}} U d^{-3 / 2}
$$

where $\gamma$ is the interfacial energy of the oil/water interface in the presence of the surfactant, $\epsilon$ is the dielectric constant of the oily phase, $\epsilon_{0}$ is the dielectric permittivity of vacuum, and $U$ is the voltage applied across the film.

The targeted electrocoalescence of droplets has been used in chapter 5, where an encap- 
sulated fibrin network formation is explored by coalescing a aqueous droplets containing a fibrinogen and a thrombin molecules.

\subsection{Droplet detection}

The real time detection of droplets is critical for the accurate control of microfluidic processes. Currently optical detection methods are the most widely used [46, 47]. The detected signal at the photo diode can be used for evaluating the droplet's size, shape, and production frequency [46]. The device can detect very high production frequencies, which are not feasible using a conventional CCD camera/microscope setup. Sheng et al. presented the capacitive detection of microfluidic droplets based on the dielectric constant contrast between the droplets and the carrier fluid, where the tested capacitance signals can be used directly for in situ labeling, sorting and droplet manipulation [47].
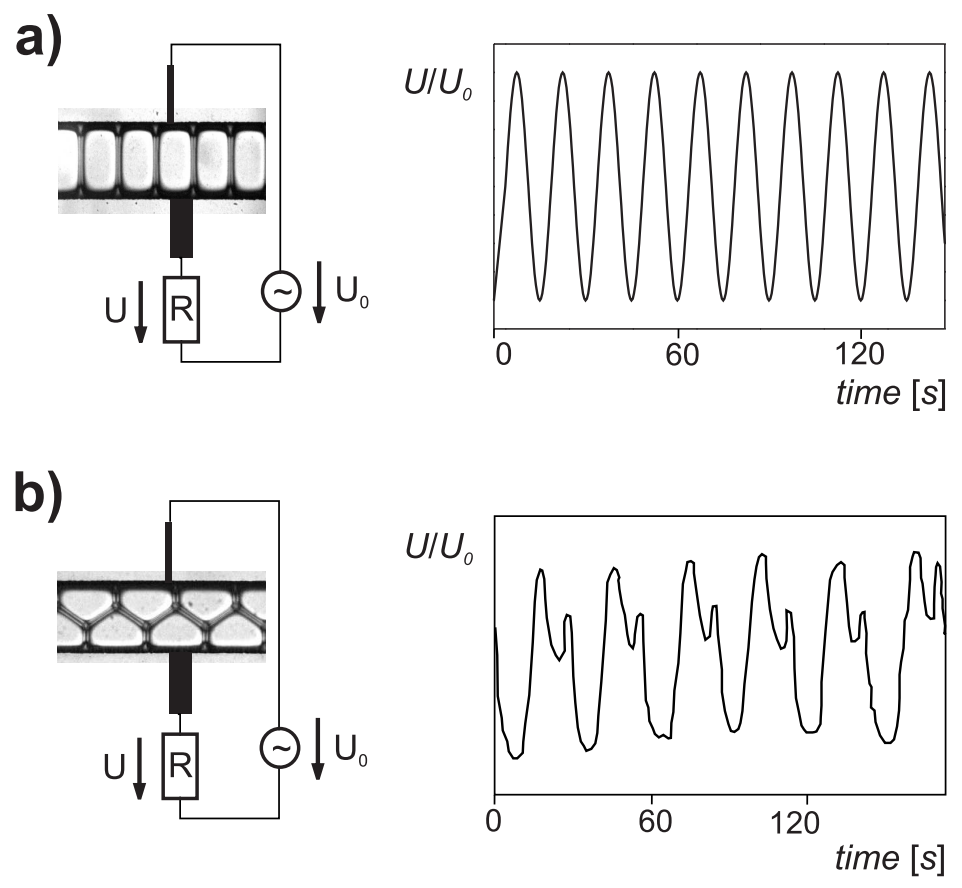

Figure 2.17: A sketch of the voltage signal $U / U_{0}$ as a function of time using two electrodes of different cross-section in contact with a) a bamboo structure; b) a zigzag structure.

For some technological applications, it may be useful to have non-optical detection of the structure flowing in a channel. When a channel width is about the same size as the 
droplet/bubble size, individual droplets of an emulsion arrange into a single file (cf. Fig. 2.17 a) which is called a 'bamboo' arrangement. If the cross section of the channel is sufficiently large so that droplets begin to evade perpendicular to the flow direction, the droplets can arrange in a 'zigzag' arrangement. Elias et al., have shown that if the foam flowing in the tube is either a 'bamboo' or a 'zigzag' structure, the structure can be detected by an electrical conductivity measurement [94] if a ferrofluid is used as a continuous phase. Due to the ionic nature of ferrofluids it is possible to perform an electrical conductivity measurements. Using two electrodes that are smaller than the separation of the Plateau borders, the detected voltage signal $U$ reflects the structure of the foam. Since the foam structures are periodic and flow within the tube at a constant velocity, the signal $U$ is periodic in time while being superimposed upon a constant signal due to a conducting wetting film of ferrofluid around the inside of the tube, cf. Fig. 2.17. A peak in the electrical signal is recorded whenever a Plateau border is in contact with one or both of the electrodes. In case of a bamboo structure both electrodes are in contact with the same Plateau border at the same time, giving a regularly spaced sequence of identical spikes in conductance (Fig. 2.17 a).

In the case of a zigzag structure, a more complicated signal can be observed. When one electrode touches a Plateau border the other electrode is only in contact with the wetting film. This leads to the occurrence of two alternating peak heights in the periodic signal (Fig. $2.17 \mathrm{~b}$ ). The small peak is recorded when the smaller electrode is in contact with a Plateau border, whereas the big peak corresponds to the case where the larger electrode is in contact with a Plateau border. For both the bamboo and zigzag structures each peak in the signal corresponds to one bubble therefore the bubbles can be counted from the electrical signal. 



\section{Chapter 3}

\section{Manipulation by Channel Geometry}

The manipulation of droplets is a critical operation that can enhance the effectiveness of droplet-based microfluidic systems which is the main topic of this thesis. Droplets can be maneuvered using both "active" and "passive" methods. "Active" methods employ external forces, e.g. movable valves [111] or electric fields [50], whereas "passive" methods employ only the channel geometry to sort, split, or re-organize the droplets. The latter has been demonstrated for foams [112] however it is not clear if the same 'rules' will apply for the emulsions since typically the volume fraction is different and a foam is compressible.

Considerably enhanced functionality in a microfluidic system can be achieved by using droplets rather than air bubbles, since droplets may contain any chemical or biological reagents of interest, which opens up an another useful aspect towards combinatorial chemistry applications [39]. Therefore there has been increasing research towards the use of individual droplets in microfluidic processing [5-10]. However, the most of these studies concentrated on a system which consisted of a low amount of the dispersed phase volume fraction so that the distance between the individual droplets was large in order to prevent unwanted coalescence. In this thesis we use the opposite approach by concentrating on controlling droplet positions in gel emulsions. Emulsions with a continuous phase volume fraction of a few percent only are called gel emulsions [113]. Their foam-like structure provides intriguing new possibilities for controlling droplet positions due to the topological interaction of the network of lamellae with the channel geometry [112, 114-116].

In the present chapter we explore the morphology of monodisperse emulsions at high dispersed phase volume fractions, which self-organize into well-defined droplet arrangements. These arrangements can be stable or metastable, depending on the geometry of the confining 
microchannel. Three arrangements are considered, in which the droplets are aligned either in a single file ('bamboo'), in a two-row ('zigzag'), or in a three-row structure. We explore the potential for induced transitions between these distinct droplet arrangements as a tool for droplet-based microfluidic processing. Furthermore, we show that neighboring relation of a monodisperse gel emulsions may be controlled in an extremely well-defined manner driven by changes in the microchannel geometry, such as a corner.

\subsection{Structural transitions}

As described in the materials and methods section, chapter 2, we dispersed Millipore ${ }^{T M}$ water in a low viscosity oil (Isopar ${ }^{T M} \mathrm{M}$, Exxon Mobil), which contains a surfactant (2 wt.\% Span 80) to stabilize the droplets against coalescence. Microchannels were fabricated using photolithography of SU8 photoresist. More detailed information about the device fabrication and emulsion type can be found in chapter 2 .

Droplets with a monodispersity better than $1.5 \%$ (coefficient of variance for droplet diameter) and with volume fractions, $\phi$, ranging from 60 to 96 vol.\% were generated either by step emulsification [44] or cross-shear using a T-piece [40, 41]. Step emulsification design offers a superior monodispersity of the droplets, while the T-junction design allows variation of droplet volume over a larger range. Therefore, whenever the volume fraction of the emulsion needs to be varied step emulsification device was applied. However, if a larger range of droplet sizes was needed, the droplets were generated by T-junction. In both T-junction and step emulsification the dispersed phase volume fraction $\phi$ can be adjusted subsequent to droplet production by injecting or subtracting continuous phase through a small side channel, whose lateral dimension was much smaller than the droplet size, see subsection 2.4.4.

In order to induce structural transitions, we must first consider what determines the droplet arrangement in a given microchannel. The main issue here is the interplay between the length scale set by the size of the droplets and the length scale and geometry of the microchannels. Depending on the droplet size to the channel cross section we obtain a different arrangements. In narrow channel, the droplets will arrange in a bamboo arrangement, cf. $3.1 \mathrm{a}$ (left) whereas when channel cross section is large enough then droplets can arrange in a zigzag arrangement, cf. $3.1 \mathrm{a}$ (middle). For a given droplet volume, both the bamboo or the zigzag arrangement might be stable within a range of channel widths depending on the volume fraction of the dispersed phase and history, i.e., the geometry of the upstream channel cross section, cf. 
Fig. $3.1 \mathrm{~b}$.

To manipulate droplet arrangements in a controlled manner, we must first consider the behavior of a monodisperse gel emulsion within the confined environment of a microchannel. The main consideration is the interfacial tension, $\gamma$, of the liquid/liquid interface, which tends to minimize the surface area of the droplets for a given channel geometry. The droplet arrangement is then largely determined by the microchannel geometry and the droplet dimension [117, 118]. Importantly, several different meta-stable arrangements may coexist, which depend on the history of the system. By means of minor channel interventions one may thus switch back and forth between different stable or metastable droplet arrangements.
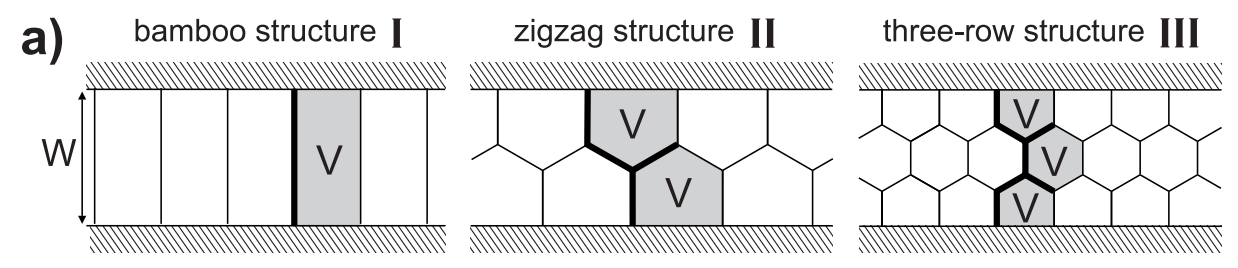

b)

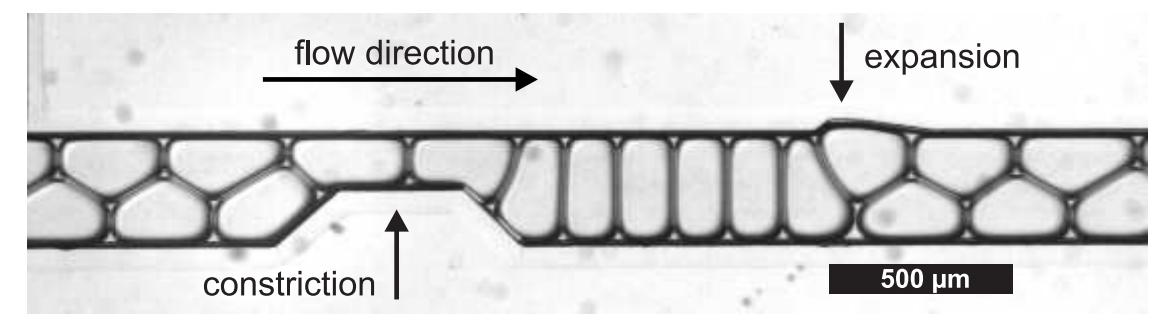

Figure 3.1: a) Sketch of the bamboo arrangement (I), the zigzag arrangement (II), and the three-row arrangement (III). b) Transition between a zigzag and a bamboo arrangement by forcing a monodisperse gel emulsion at $A^{*}=0.60$ through a channel consisting of a neck and a local expansion.

Let us first consider, as a paradigm example, transformations between the bamboo and the zigzag arrangement as they are induced by modifications in the channel geometry, see Fig. 3.1 b. For simplicity we restrict this discussion to shallow channels, where the droplet arrangement can be regarded as quasi-two-dimensional, and the shape of the lamellae is planar. The dispersed phase volume fraction is defined as:

$$
\phi=\frac{Q_{d}}{Q_{d}+Q_{c}}
$$

where $Q_{d}$ is the dispersed phase flow rate and $Q_{c}$ is the continuous phase flow rate. For 
comparison with the 2D model of an emulsion structure which will be presented here, the volume fraction will be defined as the projected 2D area fraction, as measured from the optical micrographs. In equilibrium, the intersection between three lamellae (Plateau border) is $120^{\circ}$ (see section 1.3.1). The energy contribution by the interfaces at the channel walls need not be considered since the walls always contribute the same energy, and we are interested in energy differences only. In particular, there is no need to distinguish whether or not the channel walls are wetted by the continuous phase. If each lamella has an excess free energy per unit area of $2 \gamma$ [119], we can easily express the excess free energy $E$ of each droplet in units of $\gamma W, \tilde{E}:=E / \gamma W$, where $W$ is the channel width. Similarly, the drop volume, which is the projected area of the droplet, $A$, in our quasi two dimensional consideration, can be expressed in dimensionless form as $A^{*}:=A / W^{2}$. Using these scaled quantities, we obtain $E_{\mathrm{I}}^{*}=2$ per compartment for the bamboo arrangement (dashed line in Fig. 3.2), and $E_{\mathrm{II}}^{*}=1+\sqrt{3} A^{*}$ for the zigzag arrangement (plotted as solid line in Fig. 3.2). Clearly, the zigzag arrangement is not defined any more for compartments larger than the channel width $W$. This sets an upper limit for the rescaled area, $A^{*}=\sqrt{3}$ (end point of the solid line). We find that $E_{\mathrm{I}}^{*}=E_{\mathrm{II}}^{*}$ if $A^{*}=1 / \sqrt{3} \approx 0.58$. If the droplets are smaller, the zigzag arrangement is stable, while the bamboo arrangement is meta-stable.

We can expand this theoretical consideration to three-row arrangements. Here, the excess free energy per compartment is $E_{\mathrm{III}}^{*}=2 / 3+(1+9 /(2 \sqrt{3})) A^{*}$ (dotted line in Fig. 3.2). The three-row arrangement is not defined any more if two of the Plateau borders of the middle compartments touch each other. This limits the maximum rescaled area, where three-row arrangements can exist to $A^{*}=4 \sqrt{3} /(45-18 \sqrt{3}) \approx 1.18$ (end point of the dotted line). Balancing the excess free energy per compartment for the three-row and the zigzag arrangement $E_{\mathrm{III}}^{*}=E_{\mathrm{II}}^{*}$, we find the stability condition $A^{*}=2 /(6+9 / \sqrt{3}) \approx 0.18$ for the transition between a zigzag and a three-row arrangement. If the droplets are smaller, the three-row arrangement is stable, while the zigzag arrangement is meta-stable.

In an experiment, it is in fact quite simple to switch from a zigzag arrangement to a bamboo and back again if $A^{*} \approx 1 / \sqrt{3} \approx 0.58$, i.e. close to the coexistence between a zigzag and a bamboo arrangement. The emulsion in Fig. $3.1 \mathrm{~b}$ flows from left to right, as indicated by the arrow. It is shown how a transition between a zigzag and a bamboo arrangement is achieved in an arrangement of monodisperse droplets with $A^{*} \approx 0.60$ by introducing a constriction and subsequently an expansion in the microchannel. When the gel emulsion is forced past a constriction, we move to higher $A^{*}$ in Fig. 3.2 while for an expansion we move to lower $A^{*}$. As the zigzag arrangement enters the tapered zone at the constriction, the droplets are locally 


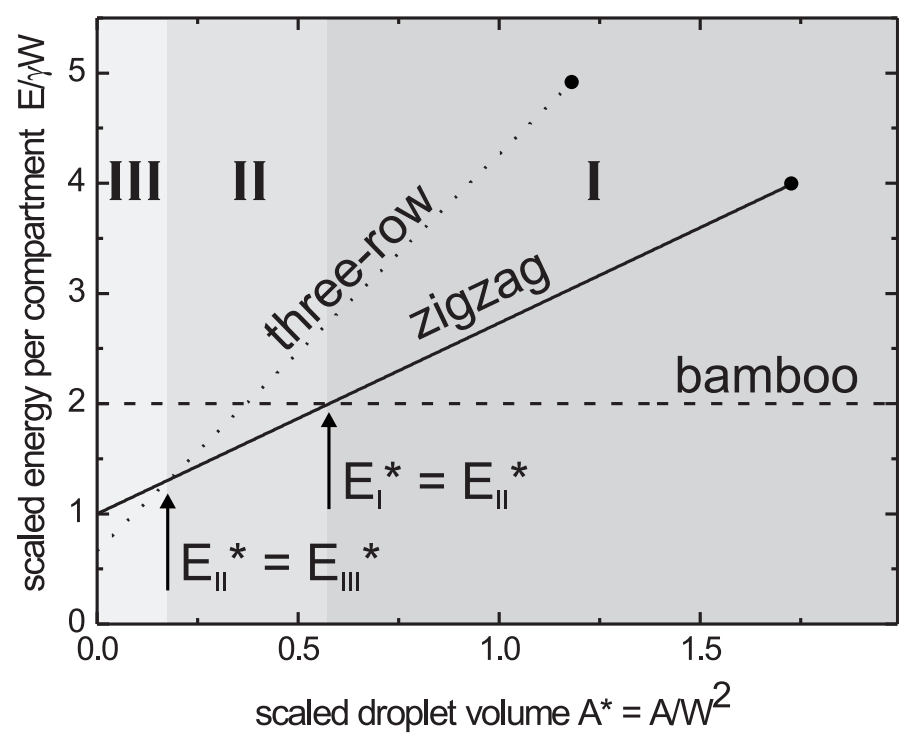

Figure 3.2: Scaled excess free Energy of the bamboo arrangement (dashed), the zigzag (solid), and the three-row (dotted) arrangement as function of the scaled droplet volume. The hatched area (I-III) denote the respective droplet arrangement with the lowest free energy.

forced into single file because they leave the region of $A^{*}$ where a zigzag arrangement can exist. After the constriction, where the channel widens, the lamellae are perpendicular to the channel walls and have no connection to one another. As the channel widens, the lamellae are simply elongated to accommodate the widening channel. At the same time, their dimension in the direction of the flow is reduced due to volume conservation, respectively area conservation in our 2d-consideration. Thereby they are shifted to region II in Fig. 3.2 where the bamboo arrangement is metastable with respect to the zigzag arrangement. Subsequently, the bamboo arrangement can be changed back to a zigzag arrangement by a local expansion in the channel width. This means we move towards even smaller $A^{*}$ in the diagram shown in Fig. 3.2. When the channel width is reduced to its original width after the expansion, the zigzag arrangement remains as the stable arrangement. The fact that for the same channel width the bamboo and the zigzag arrangement might coexist indicates that the flow direction with respect to the channel geometry is vitally important. It seems that it is important to have a steep kink at the leading edge of the expansion. However, the transition behaviour is reversible in that a constriction and expansion sequence along the length of a microchannel can periodically 
changed from one arrangement to the other, cf. Fig. $3.1 \mathrm{~b}$.

As demonstrated by this example, the transitions are hysteretic and it is therefore possible to retain the emulsion in a metastable arrangement. The hysteresis depends on the exact geometry of the constriction and the volume fraction of the gel emulsion. In the following we explore these two parameters experimentally and compare to the simple energy minimization scheme we have discussed above.

To first explore the influence of the geometry in more detail, we introduced constrictions with various well-defined geometries, cf. Fig. 3.3. The height and the upstream angle $\alpha$ of the constriction is left constant at $30^{\circ}$ whereas the downstream angle, $\beta$ is varied between $5^{\circ}$ and $75^{\circ}$. While keeping the dispersed volume fraction, $\phi$, constant at about 0.85 , we vary the droplet size for each geometry. The critical rescaled droplet volume at which the transition between a bamboo and a zigzag arrangement occurs is plotted in Fig. 3.3. For larger droplet area $A^{*}$ the droplet arrangement after the constriction is always a bamboo arrangement whereas for smaller droplet area the droplet arrangement changes back to a zigzag arrangement after the constriction as indicated by the two insets. The horizontal dashed line at $A^{*}=1 / \sqrt{3}$ indicates the theoretical stability criterion of bamboo and zigzag arrangement as derived from minimizing the surface free energy in 2D. The experimental results show that for steep obstacles $\left(\beta \gtrsim 60^{\circ}\right)$ the transition between a bamboo and a zigzag arrangement takes place where the difference in configurational energy changes sign. This is expected since a steep slope provides sufficient disturbance to prevent the system from being trapped in a metastable state. For down stream angles $\lesssim 45^{\circ}$, however, we find a clear departure from this behavior, indicating the survival of the metastable state: the smaller the downstream constriction angle, $\beta$, the more easily a bamboo arrangement is stabilized downstream from the constriction.

Besides the precise geometry of the constriction, the hysteretic behavior also depends on the volume fraction $\phi$ of the dispersed phase [112]. In Fig. 3.4 the hysteretic topological rearrangement is shown for a constant droplet size as a function of volume fraction of the gel emulsion. For the tapered channel shown, we determined the rescaled channel widths $W / \sqrt{A}=1 / A^{*}$ at which the transitions between the bamboo, the zigzag, and the three-row arrangement occur. Comparing the transition points for the opening taper with those for the closing taper, we clearly see that there is considerable hysteresis. This hysteresis is found to increase with the volume fraction of the dispersed phase and becomes noticeable for $\phi>0.80$ in agreement with experimental results using foams on the mm-length scale [112]. Consequently, the probability to retain a particular droplet arrangement increases with increasing $\phi$. The physical reason for this hysteretic behavior is the finite energy required for topological 
a)
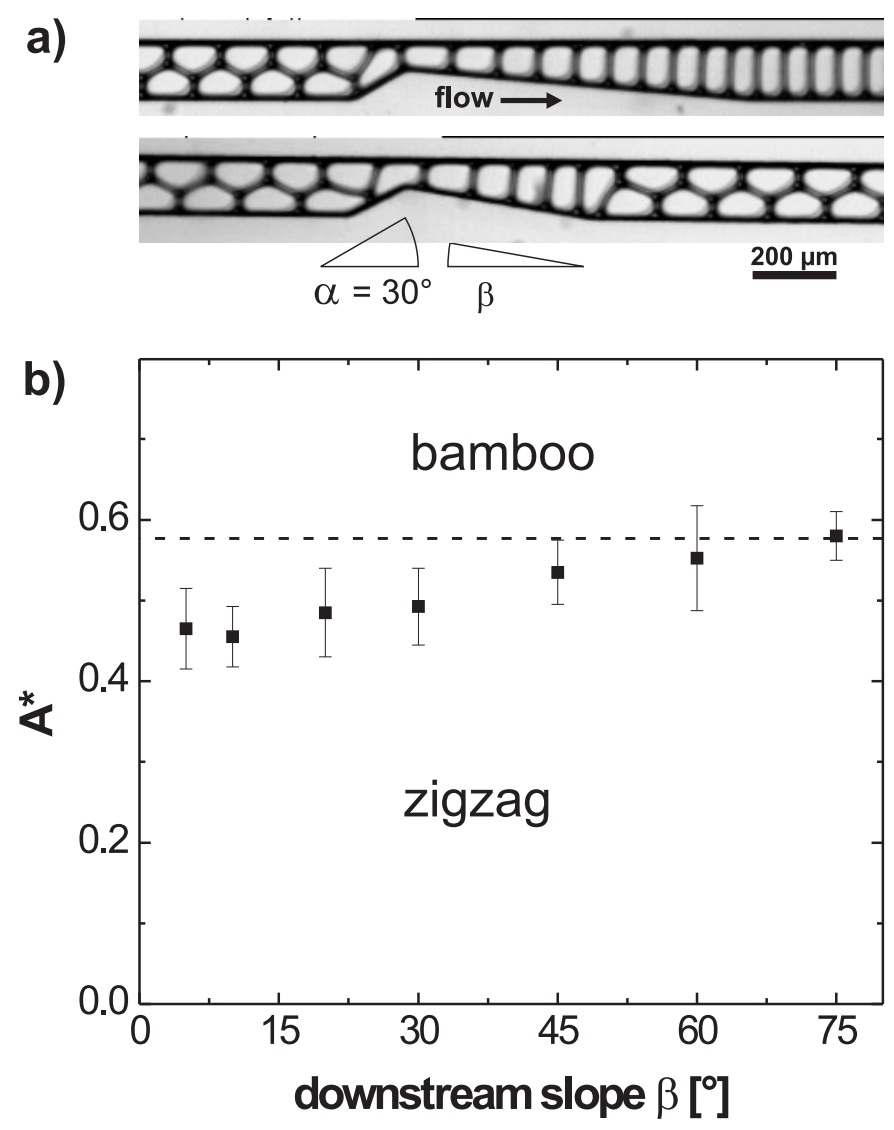

Figure 3.3: Gel emulsions with the same droplet area flowing from left to right through different channel geometries. Top: the bamboo arrangement remains in the metastable configuration $\left(\beta=5^{\circ}\right)$. Bottom: the bamboo arrangement changes back to the zigzag arrangement $\left(\beta=10^{\circ}\right)$. b) Transition between a zigzag and a bamboo arrangement by suitable channel geometries. Stability diagram showing the critical scaled droplet volume $A^{*}=A / W^{2}$, where either a bamboo [I] or a zigzag [II] arrangement is found as a function of the downstream slope angle, $\beta$ of the feature. The dashed line corresponds to the simple geometrical model, $A^{*}=1 / \sqrt{3}$.

transitions of the foam-like network. One example is the T1 transition [90-93], where two vertices of the foam (Plateau borders) merge and separate again to form a different bubble/droplet arrangement (cf. section 1.3.1 and Fig. 1.5).

Fig. $3.4 \mathrm{~b}$ shows the hysteresis effects in more detail. The interval spanned between open and closed symbols of the same type indicate regions of coexisting zigzag and three-row (triangles) or bamboo and zigzag (squares) arrangements. The horizontal lines at $1 / \sqrt{A^{*}}=1.32$ 
a)

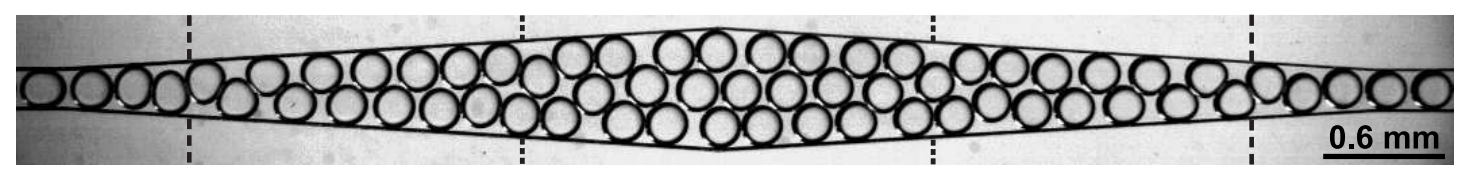

$\triangle$

$\triangle$
[I] to [II]
[II] to [III]
[III] to [II]
[II] to [I]
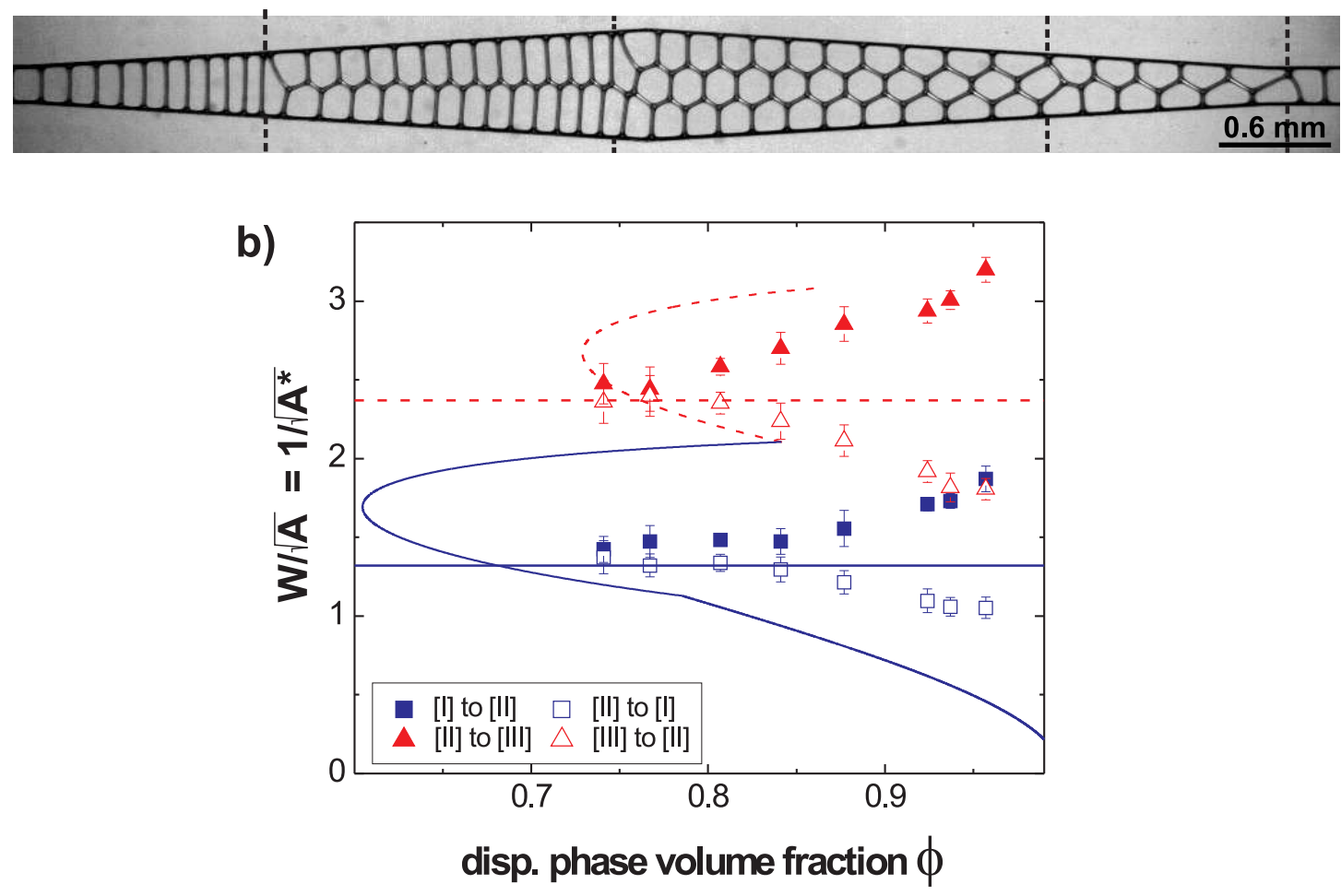

Figure 3.4: a) Optical micrographs: monodisperse gel emulsions flowing through a wedge shaped channel.(top: $\phi=0.74$, bottom: $\phi=0.96$ ). The dashed lines mark the position of the respective transitions. b) Regions of coexisting different droplet arrangements, as found experimentally. Triangles: zigzag/three-row coexistence. Squares: bamboo/zigzag coexistence. Horizontal lines: normalized channel widths corresponding to the intersection points in Fig. 3.2. The curved lines denote the transition for hard discs between [I] and [II] (solid) and [II] and [III] (dashed), respectively [120].

and $1 / \sqrt{A^{*}}=2.37$ correspond to the points of intersection in Fig. 3.2 as obtained from the 2D geometrical foam model. It is interesting to note that these lines agree well with the experimental results for $\phi<0.80$, although the droplets have almost undisturbed circular shapes in this regime. This can be well appreciated from the upper panel in Fig. $3.4 \mathrm{a}$ for $\phi=0.74$, where the droplets barely touch one another. 
In fact, we can use the hard disks model to describe the behavior at low values of $\phi$ [120]. The curves in Fig. $3.4 \mathrm{~b}$ indicate the maximum dispersed phase volume fraction which can be obtained by packing hard disks with a preset value of $A^{*}$. We see a rough agreement of the lobes of these curves with the coexistence regions. The significant under estimation of $\phi$ is not unexpected due to the deformability of the droplets.

For comparison, we plotted the calculated packing behavior of solid (undeformable) discs [120] in Fig. 3.4 b. The solid and the dashed curves denote the transitions between a bamboo and a zigzag arrangement of solid discs and the transition between a zigzag and a three row arrangement, respectively. Qualitatively, the hysteretic behavior is resembled by this theory. However, quantitatively there is still an open question for the theory regarding $2 \mathrm{D}$ emulsion arrangement i.e. deformable discs, as a function of the volume fraction. As expected, the behavior of solid discs provide a lower bound concerning the volume fraction; The experimental transitions take place at significantly larger $\phi$. This shows that they are determined by the geometrical arrangement of the compressed cells, or by Plateau borders.

\subsection{Droplet reorganizations at a corner}

When droplets are monodisperse and produced at high volume fraction, each droplet's position is well-defined relative to its neighbors. By addressing individual droplets in real time, the complex processing of chemicals (e.g. for synthesis and analysis) and bio-matter (e.g. cell viability, protein crystallization) can be achieved. Indeed, the positions of the droplets may be precisely manipulated by introducing a corner in the channel geometry as shown in Fig. 3.5 . We prepared microchannels incorporating a sequence of corners of varying corner angle $\alpha$. These bends were not smooth $180^{\circ}$ bends as studied elsewhere [112], but rather two straight channels that meet with a certain angle. By following the reorganization through each corner, the phase shift of droplets relative to their neighbors was identified [121].

The different reorganizations are summarized in Fig. 3.5 where the droplets next neighbor relation is indicated before and after the corner. The droplets in the outer and inner row are marked black and white respectively. For the $160^{\circ}$ corner (Fig. 3.5 a) no phase shift was observed, which means that the next neighbor relation of the droplets before and after the corner was identical. For the slightly sharper corner of $120^{\circ}$, the inner row of droplets (white) moved one position ahead of the outer row of droplets (black), cf. Fig. 3.5 b. For acute angles the number of phase shifts increase; two positions at $40^{\circ}$, cf. Fig. $3.5 \mathrm{c}$, and three positions at 
$20^{\circ}$ (images not shown). For acute angles, droplets were permanently captured in the corner (marked in yellow). The more acute the angle, the greater the number of captured droplets. In Fig. 3.6 a three-row zigzag arrangement flowing through a bend channel is shown. Here we found similar behavior to that observed in the case of the two-row zigzag arrangement. However, due to the simplicity, more quantitative investigations were carried out for the tworow zigzag arrangements. This geometric constraint is flow rate independent which was a finding of in our experiments. This result is in contrast to translocation in a smooth $180^{\circ}$ bend [112] where friction at the channel walls was found to be crucial.
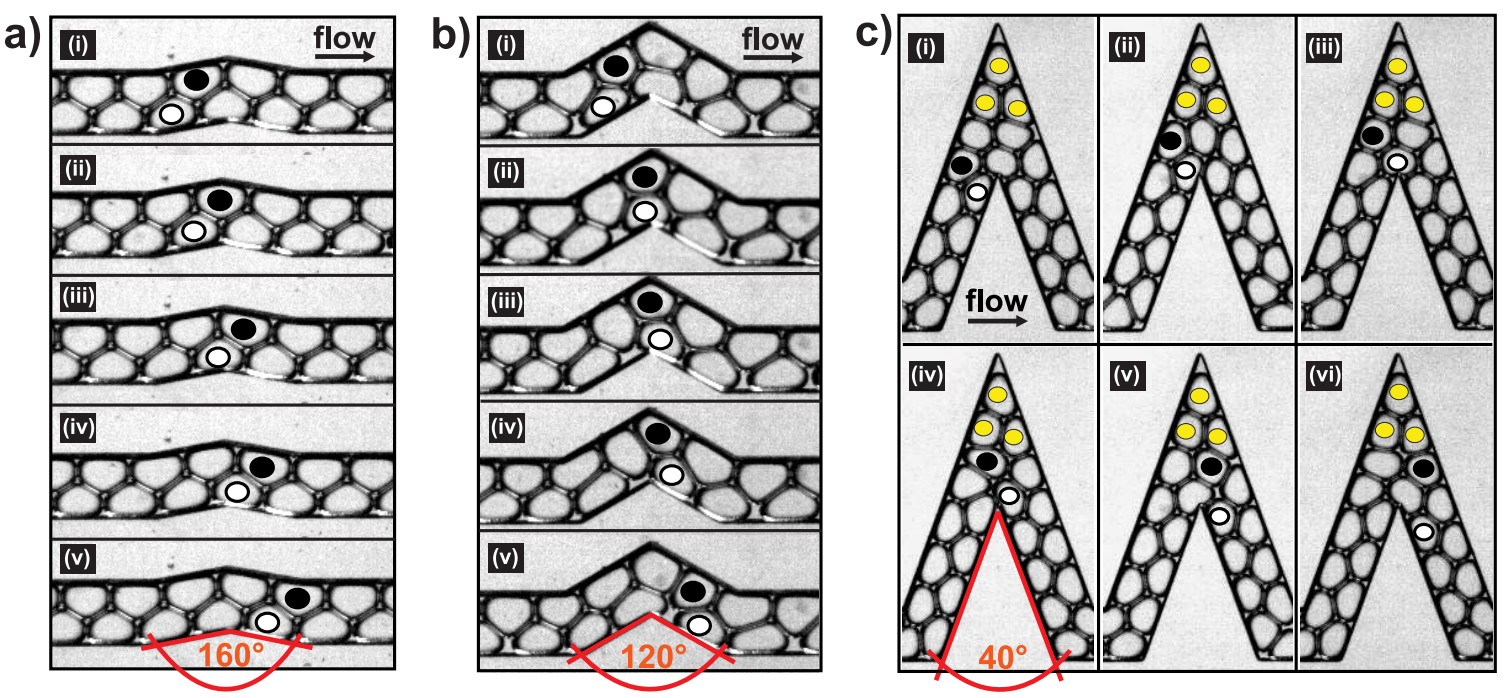

Figure 3.5: An example of two-row zigzag arrangements with a volume fraction of $\phi=0.81$ flowing along a corner. Depending on the corner angle the relative position may changed by up to three positions. a) For the $160^{\circ}$ corner, no phase shift was observed. b) a $120^{\circ}$ corner induced a one position phase shift with respect to the outer row of droplets. c) For a more acute angle $\left(40^{\circ}\right)$, a two position phase shift was induced. Droplets marked yellow were permanently captured in the corner. The channel width is $280 \mu \mathrm{m}$.

The phase shift of the droplets through the corner can be explained by considering the path length of the inner and outer row of droplets travelling through corners as shown in Fig. 3.7 a. We calculate the geometric path length $\Delta$ (The length of the red line in Fig. 3.7 a) between the $1^{\text {st }}$ and the $n^{\text {th }}$ row taken at the respective center of the droplets in units of the width of a single droplet ("unit cell"). Thus, the phase shift in units of a unit cell length can be defined as: $\delta:=\Delta / d$, where $d$ is the droplet width. By defining the angular deviation from a straight channel by $\beta:=\pi-\alpha$, we obtain for the phase shift $\delta:=\frac{1}{A^{*}} \tan (\beta / 2)$. Here $A^{*}$ is the normalized 


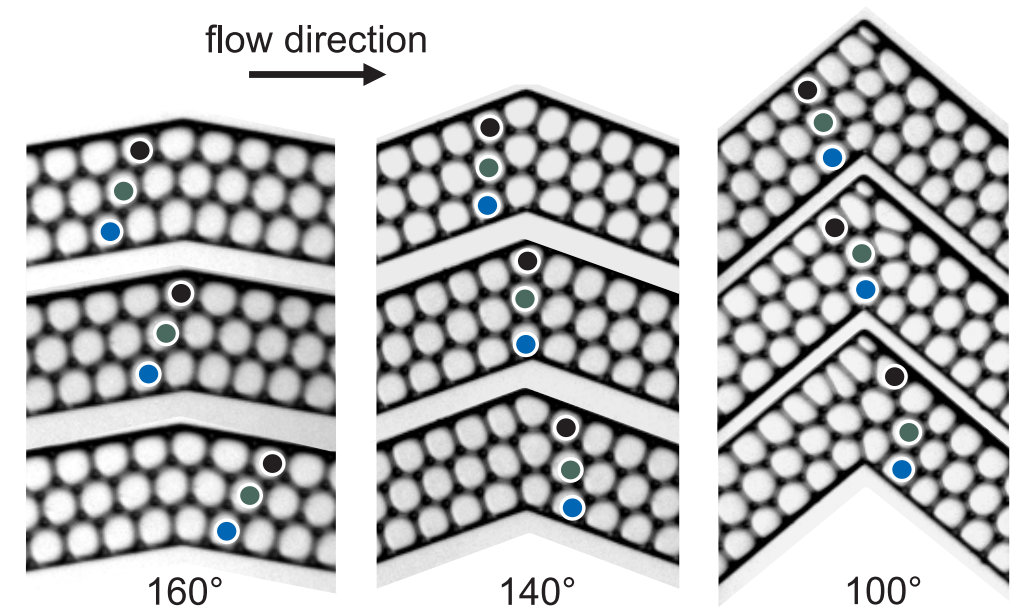

Figure 3.6: Three-row zigzag arrangements in the corner channel. As the case of a two-row zigzag arrangement at a $160^{\circ}$ corner, there was no phase shift observed. For the $140^{\circ}$ corner, a one position phase shift was induced between the $1^{\text {st }}$ and $2^{\text {nd }}$ row and the $2^{\text {nd }}$ and $3^{\text {rd }}$ row, respectively. For a more acute angle $\left(100^{\circ}\right)$, a one position phase shift was induced between the $1^{\text {st }}$ and the $2^{\text {nd }}$ row, while there was two phase shifts induced between the $2^{\text {nd }}$ and the $3^{r d}$ row.

$2 \mathrm{D}$ volume (area) of the droplet that can be expressed as $A^{*}:=A / W^{2}$. To calculate the center of mass of the droplet we approximated the shape of the droplet as rectangular, which does not change the center of mass compared to hexagonal droplets, but allowed us to account for different volume fractions. In the calculation of the width of the unit cell we used an increased

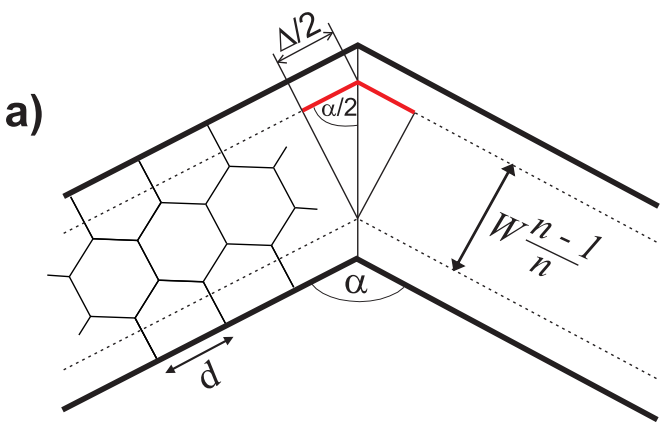

b)

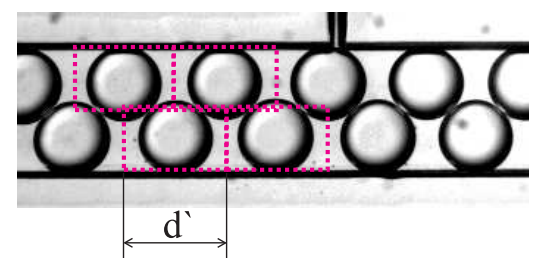

Figure 3.7: a) A schematic of path lengths for the inner and outer rows of droplets around the corner. The path length difference at the center of the droplets, $\Delta$, is indicated by the red line. b) A sketch of a unit cell length $\left(d^{\prime}\right)$ used in the model. 
droplet width $d^{\prime}$ i.e. the droplet width $d$ plus the lamellae thickness, see Fig. 3.7 b. Assuming that the continuous phase is just a line, then the increased drop volume accounts for the remaining continuous phase.

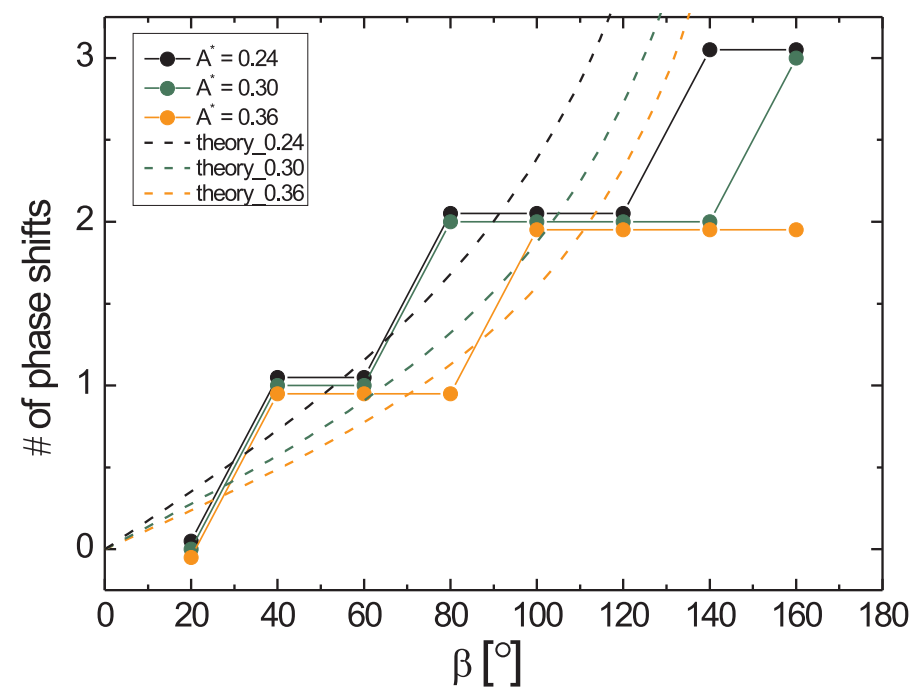

Figure 3.8: Number of phase shifts as a function of angle, $\beta$ for various drop sizes. The angle $\beta$ is the angle of deviation from the straight channel. The dispersed phase volume fraction was $\phi=0.92$.

To systematically investigate the neighboring relation of droplets more quantitatively, we considered a number of parameters like the drop size and the dispersed phase volume fraction. The number of phase shift as a function of angle $\beta$, where $\beta$ is the deviation angle from the straight channel $(\beta=(\pi-\alpha))$ for various droplet sizes are shown in Fig. 3.8. Here the dispersed phase volume fraction is kept constant at $\phi=0.92$. As we can see from Fig. 3.8 smaller droplets induce a greater number of phase shifts. The dashed lines represent our simple theoretical consideration, based on the relative path length of the inner and outer rows of droplets whereas the data points represent our experimental results. The theoretical assumptions are in good agreement with the experimental results in general. However, at acute angle, the experimental data points diverge from the theoretical results especially for bigger droplet sizes (the orange data points in Fig. 3.8). If repeat these experiments for a lower dispersed volume fraction, see Fig. 3.9, more acute corners are needed to achieve the same number of phase 
shifts as compared with the higher dispersed volume fraction.

In Fig. 3.10, we combined the results of several measurements such as those shown in Fig. 3.8 and Fig. 3.9 for various volume fractions together with the prediction from our simple model. Here the number of the phase shifts are plotted as a function of angle, $\beta$ for various volume fractions at fixed drop size. It was observed that the higher the volume fraction, the larger the number of phase shifts induced by the corner. For small $\phi$ (the blue data points, $\phi=0.67$ in Fig. 3.10 ) it seems the experiment rather escape to lower number of phase shift whereas for larger $\phi$ (the black data points, $\phi=0.92$ in Fig. 3.10), it seems to escape to the larger number of phase shift.

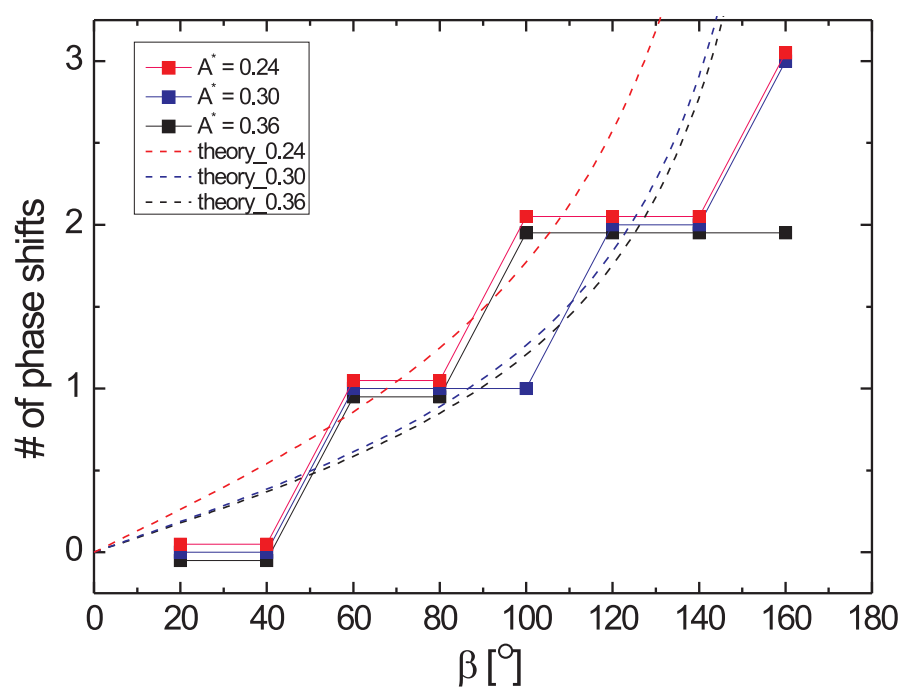

Figure 3.9: Same as Fig. 3.8, but for dispersed phase volume fraction of $\phi=0.67$.

To relate a critical drop size with the number of phase shifts we fixed the corner angle at $90^{\circ}$ and the dispersed volume fraction to $\phi=0.85$, while the drop size $A^{*}$ was varied between 0.15 and 0.60. This is the maximum range in drop size where a zigzag arrangement can be found as explained in the previous section (pp. 46 and 47). In Fig. 3.11 two examples are shown; In Fig. 3.11 a the drop size was $A^{*}=0.19$ and the number of phase shifts were found to be two. In Fig. 3.11 b, the drop size was almost doubled to $A^{*}=0.34$ and the number of phase shifts was one. By examining our experimental data, we found the critical volume of droplet to be $\approx 0.28$ for the given parameters $\alpha=90^{\circ} ; \phi=0.85$. The critical drop size for a certain 
number of phase shift, $A_{c}^{*}$ will vary depending on the corner angle and the volume fraction of the emulsion. For $A^{*}<0.28$ we expect two or more droplet shifts, and for $A^{*}>0.28$ we only expect to get one droplet shift.

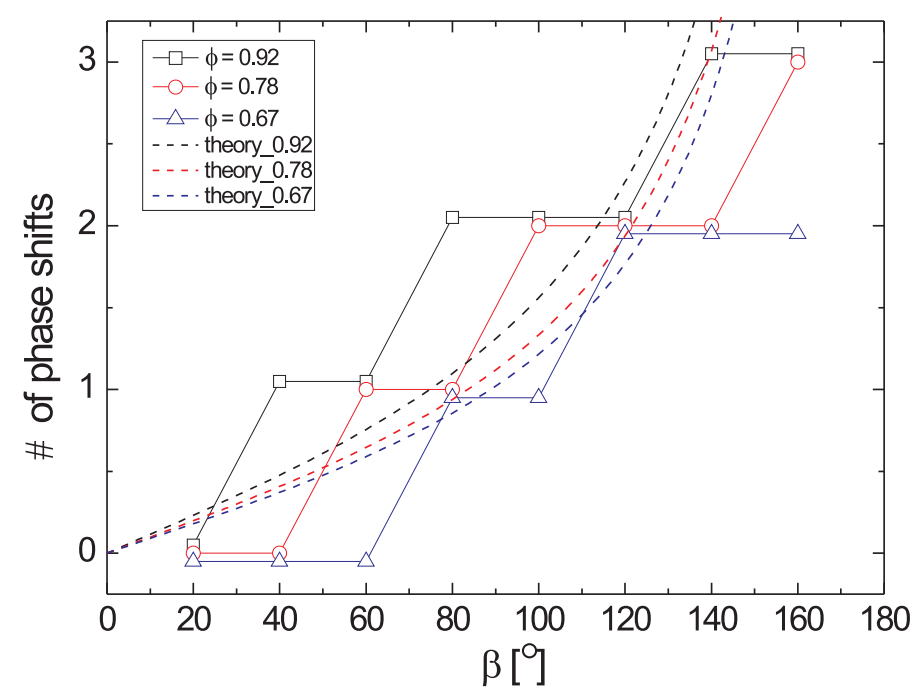

Figure 3.10: Number of phase shifts as a function of angle, $\beta$ for various dispersed volume fractions. The normalized volume of the drop is $A^{*}=0.36$.

a)

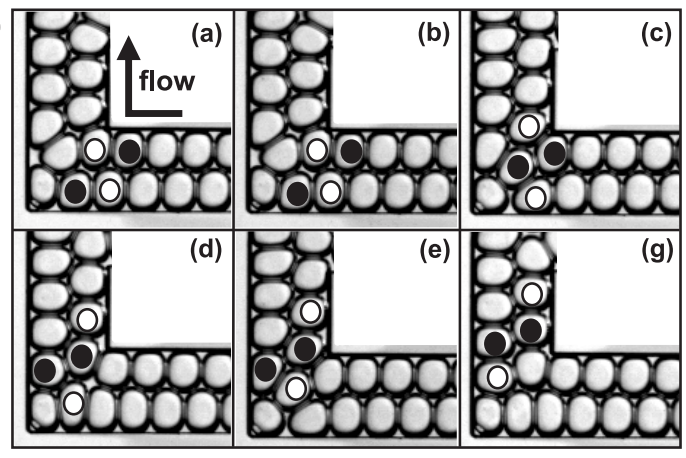

b)

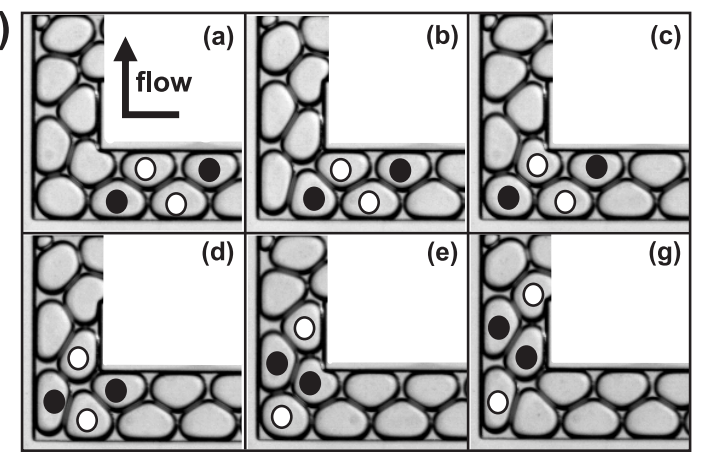

Figure 3.11: With a fixed corner angle of $90^{\circ}$ and a volume fraction of the dispersed phase of $\phi=0.85$, only the drop size, $A^{*}$ is varied. a) When $A^{*}=0.19$, the number of phase shifts were two. b) When $A^{*}=0.34$, the number of phase shifts was one. In both cases droplets droplets that are initially separated (marked black) before the corner come into contact after they had passed the corner. 
The controlled mechanism to change the neighboring relations might be used in combination with e.g. electrocoalescence for combinatorial microfluidic applications. In Fig. 3.11 four droplets are labeled before and after the $90^{\circ}$ corner. Originally, the black droplets are separated by two white marked droplets in a zigzag arrangement (Fig. $3.11 \mathrm{a}(\mathrm{a})$ ). The droplet positions are fixed until they enter the corner (Fig. $3.11 \mathrm{a}(\mathrm{b})$ ), where a one-position phase shift brings the once separated droplets together (Fig. $3.11 \mathrm{a}(\mathrm{d})$ ). This behavior may be reversed (by a second corner) or may be used to position droplets relative to one another for electrocoalescence (i.e. to initiate a reaction).

This mechanism where the outer two droplets come into contact after passing the corner, is observed whenever the number of phase shifts is one or two and is independent of the corner angle. For the smaller droplets the number of phase shifts are two, whereas in case of larger droplets, the number of phase shifts is just one. In this case, the only difference is those two inner droplets may exchange positions after they had passed corner therefore giving a different result for the final number of phase shifts. When the number of phase shifts is three then the outer two droplets can not get in contact e.g there will be another droplet in between. This example demonstrates, how precisely one can manipulate the each individual droplet positions with respect to the neighboring droplets.

The observed deviations of our experimental results to the model based on the geometric path length suggest that the number of phase shifts is not additive if we combine several corners of different angles. Let's take the example of $\phi=0.92$ from Fig. 3.10 and consider the number of phase shift for a full $360^{\circ}$ circle.

$$
\begin{aligned}
& 18 \times 20^{\circ} \text { corner } \Rightarrow 0 \text { phase shifts } \\
& 9 \times 40^{\circ} \text { corner } \Rightarrow 9 \text { phase shifts } \\
& 6 \times 60^{\circ} \text { corner } \Rightarrow 6 \text { phase shifts } \\
& 4.5 \times 80^{\circ} \text { corner } \Rightarrow 9 \text { phase shifts } \\
& 3.6 \times 100^{\circ} \text { corner } \Rightarrow 7.2 \text { phase shifts } \\
& 3 \times 120^{\circ} \text { corner } \Rightarrow 6 \text { phase shifts }
\end{aligned}
$$

If we construct the full circle using a $40^{\circ}$ corner 9 times we expect 9 phase shifts, while we expect no phase shifts in the case of a $20^{\circ}$ corner 18 times. This result suggests that the phase shifts can be maintained if appropriately shaped corners are inserted along a straight channel, respectively the number of phase shifts might be continuously increased by inserting several such corners into a straight channel. This may be applied to combinatorial multistep reactions where the neighbouring droplets have to be exchanged. 



\section{Chapter 4}

\section{Manipulation by Magnetic Fields}

In the previous chapter we have seen that the arrangement of droplets in a microchannel can be changed by geometrical constrictions. Since a different droplet arrangements might change the result of a Y-junction, cf. Fig. 4.1 it might be very interesting for microfluidic processing to manipulate the geometry of the constriction on demand by external fields. Actively switching between a bamboo and a zigzag arrangement might allow online sorting, dividing, and other microfluidic processes. To explore the active switching we have generated aqueous droplets in a ferrofluid as the continuous phase, which can be manipulated applying a magnetic field.

Ferrofluids have novel properties and their behavior in magnetic fields have led to many interesting applications. Engineering applications of ferrofluids in microflidics rely upon their basic properties such as retention in a magnetic field, levitation of magnetic and non-magnetic objects, micromagnetics and catalysis associated with small particles, magnetic shielding and their effect on electrical parameters [122]. A review paper by Pamme [123] describes the various developments within the field of magnetism and microfluidics. Plugs composed of a ferrofluid and transported by external magnetic fields can be used for pumping liquids through channels. Hatch et al. fabricated a circular ferrofluidic micropump for continuous pumping of water [124]. Hartshorne et al. reported the use of a ferrofluid as a valve in a microchannel [125]. Water based ferrofluids can be made bio-compatible, rendering them useful for many biomedical applications such as cell manipulation and sorting schemes [126, 127]. Ferrofluidic foams, i.e. ferrofluids with air bubbles, have also been investigated [94, 112, 115].

As we discussed in the previous chapter, for a given droplet volume, both the bamboo or the zigzag arrangement might be stable within a range of channel widths depending on the volume fraction of the dispersed phase and history, i.e. the geometry of the upstream channel 

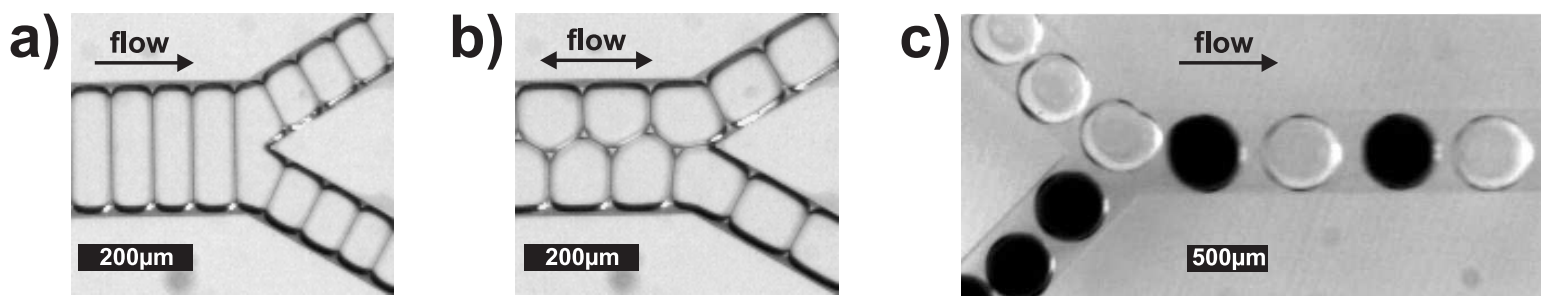

Figure 4.1: Optical micrographs of monodisperse gel emulsions in microfluidic channels a) When droplets of a bamboo arrangement arrive at the Y-junction they are split in two smaller droplets. b) When a zigzag arrangement of droplets arrive at a Y-junction the two droplet rows are reversibly separated or combined depending on the flow direction. c) Alternating droplets of different type (black droplets contain fluorescent dye, Eosin B) using a Y-junction [45].

cross section. This dependence on history is most easily appreciated considering a simple Y-junction. It can either act as a 'zipper', separating or combining two droplet rows reversibly (cf. Fig. $4.1 \mathrm{~b}$ ), or it can split the droplets when they arrive as a bamboo structure (cf. Fig. 4.1 a). By changing the arrangement of the droplets (bamboo or zigzag) we can alter the operation of the Y-junction. Using a Y-junction, droplets that contain different chemicals can be alternated in a downstream channel, cf. Fig. $4.1 \mathrm{c}$. It is however, the initial emulsion arrangement that determines the consequence of a Y-junction and therefore precise manipulation of this arrangement is very important.

\subsection{Water-in-ferrofluid emulsions in capillaries}

We used a PMMA device with either T-junction or step emulsification unit in the microchannel (see chapter 2.4). This channel is connected to a capillary with circular cross section, whose diameter is larger than the channel width. Therefore, the droplets are packed close to one other in the capillary. For various drop sizes several different emulsion arrangements can be observed, cf. Fig. 4.2. The arrangement depends on the ratio of the capillary diameter to the droplet diameter and becomes complex if this ratio is very large, as can be seen from the Fig. $4.2 \mathrm{c}$ and Fig. 4.2 d. For simplicity we only consider about the bamboo and zigzag arrangements in our experiments. The volume fraction of the emulsion is also important for the droplet arrangement; The higher the volume fraction, the more the ordered the arrangement becomes. These droplet arrangements in the capillary can be manipulated by external magnetic field. We used permanent magnets of different sizes to apply magnetic field gra- 
dients. A magnetic field gradient can twist the arrangement, or induce the transition from one arrangement to another cf. Fig. 4.3 depending on the strength and the lateral extension of the magnet. Applying a magnetic field using a small pole shoe, rotates the arrangement (or twist) cf. Fig. 4.3 a, whereas a large pole shoe induces a transition between the zigzag and bamboo arrangement cf. Fig. $4.3 \mathrm{c}$.

(a)

(b)

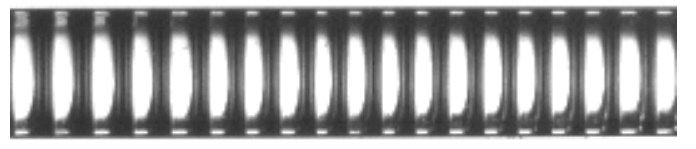

(c)

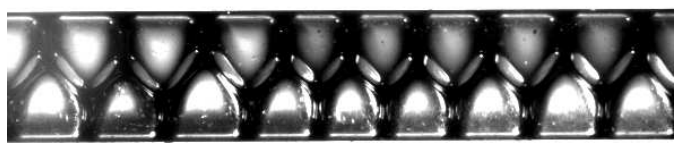

(d)
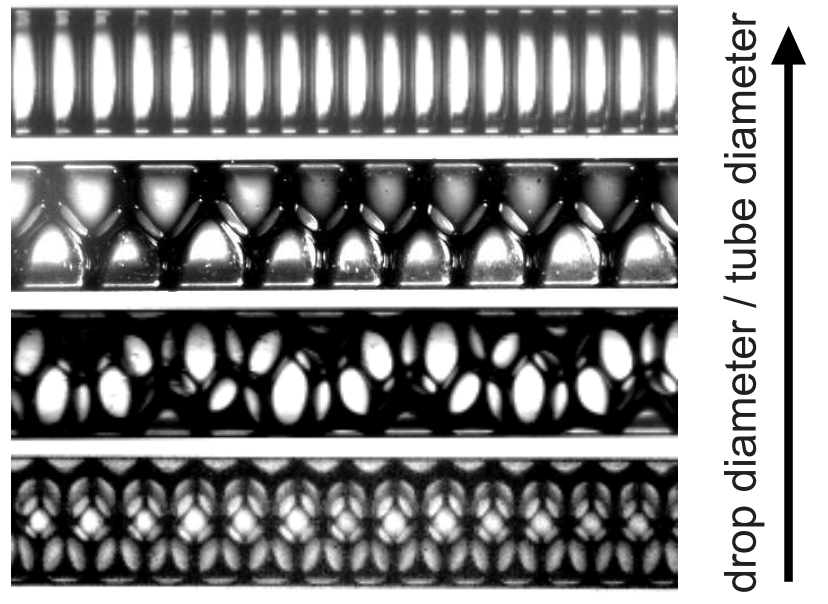

Figure 4.2: Ordered emulsion arrangements in a capillaries. a) bamboo, b) two-row zigzag, c) three-row, d) four-row structure. The topology of the droplet arrangement depends on the ratio of the droplet diameter to capillary diameter.

As what we found in the previous chapter, stability of a bamboo and zigzag arrangements are important whether a transformation between those arrangements will happen. In other words, if a zigzag arrangement is in a metastable region, it is quite easy to transform it from the zigzag arrangement to the bamboo arrangement applying a magnetic field. This can be seen from Fig. 4.3. In the case of smaller droplets, the zigzag arrangement remains the same after the pole shoe (cf. Fig. 4.3 b), while the emulsion arrangement containing slightly bigger droplets is transformed into a bamboo arrangement (cf. Fig. $4.3 \mathrm{c}$ ) with the same applied field strength and distribution. In all cases, an accumulated ferrofluid plug is observed at the location of highest magnetic field strength due to the high magnetic susceptibility of the ferrofluid. These findings are in good agreement with a ferrofluid foam systems on larger length scale [112].

Due to rotational invariance of the droplet arrangements in a capillary with a circular cross section, the rearrangements of the droplets is very difficult to observe. For quantitative experiments we thus used microfluidic devices with rectangular cross section and vary the magnetic 
field distribution by various pole shoes of an electromagnet that allows to adjust the magnetic field strength continuously.

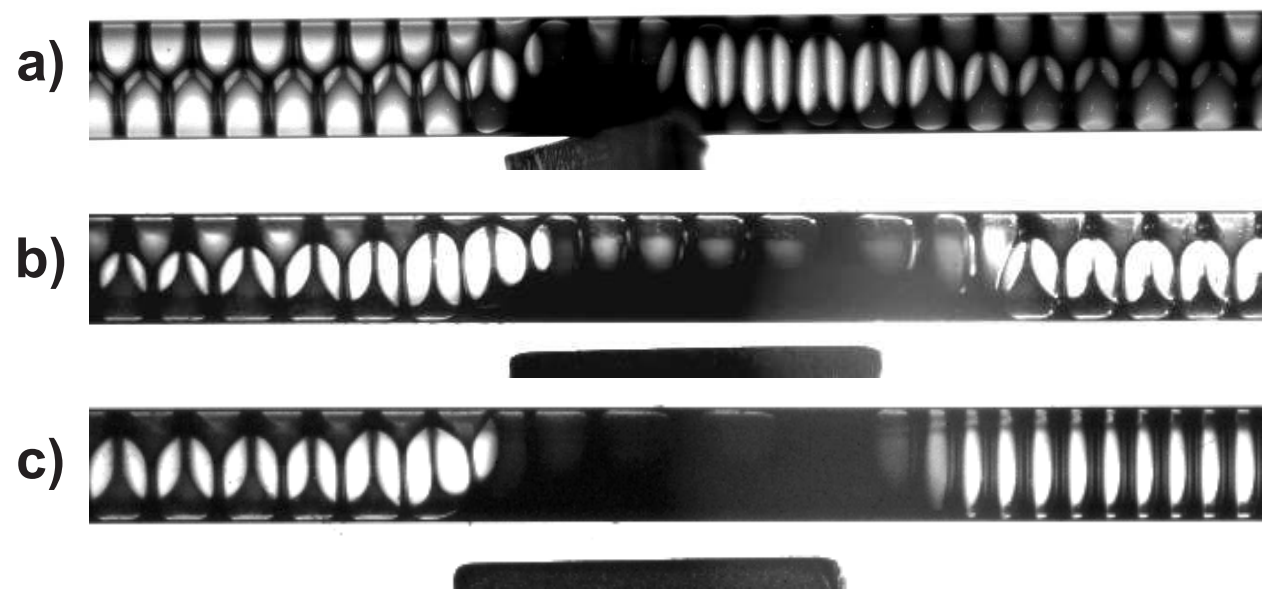

Figure 4.3: Droplet manipulation in a cylindrical capillary by inhomogeneous magnetic field using a permanent magnets $(\mathrm{NdFeB})$ and a ferrofluid as the continuous phase. The pole shoe is visible at the bottom of each image. a) Arrangement rotation using a small pole shoe b) A large pole shoe accumulates more ferrofluid but no transition occurred c) When slightly larger droplets are imposed, the transition from zigzag to bamboo arrangement observed.

\subsection{Water-in-ferrofluid emulsions in microchannels}

As described in section 2.2.1. microchannels were directly micro-machined in a PMMA block and were closed by a PMMA cover plate. The microfluidic chip is cut from the side in order to bring the pole shoe of the electromagnets close to the channel cf. Fig. 2.3 . Both T-junction (see chapter 2.3.1) and step emulsification (see chapter 2.3.3) techniques were used to generate emulsions. T-junction device was used to generate a wide range of droplet sizes whereas step emulsification device allows to produce a high dispersed phase volume fraction of emulsion. In order to manipulate water-in-ferrofluid emulsions in the microchannel, we used an electromagnet (Model 3470, GMW Associates, San Carlos, California, USA) capable of producing a magnetic field strength of up to a $1.8 \mathrm{~T}$ cf. Fig. 4.4. In order to get uniform magnetic fields, we used a symmetric pole face ( $45 \mathrm{~mm}$ diameter) and, to generate inhomogeneous magnetic fields, we used a single pointed pole shoe $\left(3.5 \mathrm{~mm}\right.$ diameter, $77.5^{\circ}$ cone angle). The magnetic field strength in the microchannel was measured by a Hall probe (Model 
907, Messtechnik and Electronik $\mathrm{GmbH}$ ) as shown in Fig. 4.4. Water cooling of the magnet is possible when using high magnetic fields for a long time, but was not needed in the experiments described here.

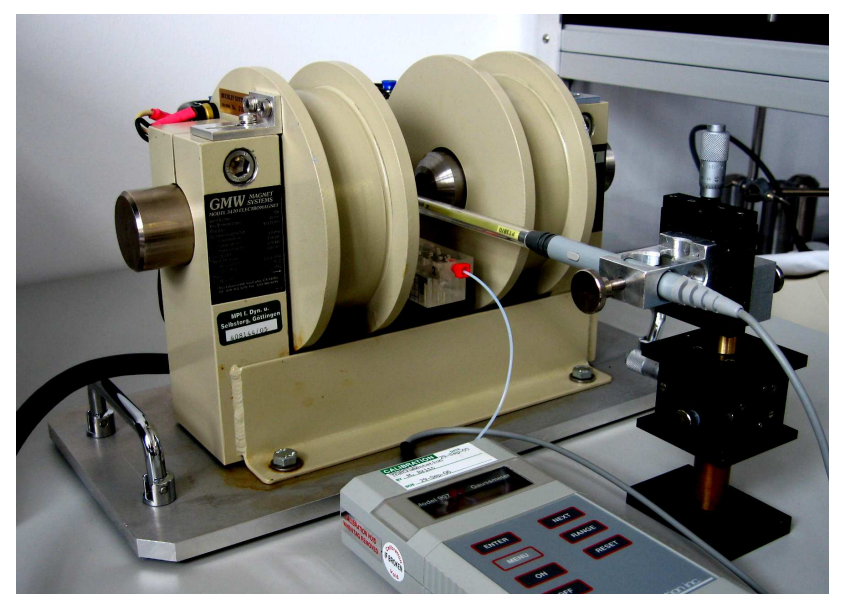

Figure 4.4: The electromagnet used in our experiments, which is capable of producing magnetic fields up to $1.8 \mathrm{~T}$. The hall probe mounted on the micromanipulator was used to determine the local field strength.

We applied a homogeneous magnetic field using a large, rotational symmetric pole shoe with a radius of $r=22.5 \mathrm{~mm}$ (cf. Fig. 4.5 ). In Fig. 4.5 b, the normalized magnetic field strength is displayed as a function of the lateral distance from the center of the pole shoe. The shape of the pole face is sketched at the bottom of the graph in light grey, in Fig. $4.5 \mathrm{~b}$. The microfluidic channel containing an emulsion with a zigzag arrangement was placed in the middle of the pole shoes at a distance $\approx 2.5 \mathrm{~mm}$ from the center of the pole shoe at an applied magnetic field of up to $1.5 \mathrm{~T}$. In this area the magnetic field is homogeneous and as expected [95], the ferrofluid lamellae do not align parallel to the homogeneous magnetic field and no changes in the droplet arrangement could be detected, cf. Fig. 4.5 a.

Alternatively, when applying an inhomogeneous magnetic field using a pointed pole shoe with a radius of $r=1.75 \mathrm{~mm}$, the ferrofluid emulsion arriving in a zigzag arrangement forms a temporarily variable "plug" of ferrofluid. Using this inhomogeneous field distribution, the emulsion can be switched from a zigzag to a bamboo arrangement, cf. Fig. 4.6. The dynamic evolution of the ferrofluid plug is displayed in Fig. 4.6. When switching on the magnetic field, the continuous phase consisting of ferrofluid, which are close to the pole shoe grow thicker as can be seen from the second image (at $\mathrm{t}=2 \mathrm{~s}$ ) in Fig. 4.6. One can see the pole shoe of the 
electromagnet at the bottom of the image which is brought close to the microfluidic channel cf. Fig. 4.6. With time the lateral extension of the ferrofluid plug increases. Therefore, droplets have to align in a single file to accommodate in the environment where channel width is decreased when passing the ferrofluidic accumulation. Thus, the zigzag arrangement transforms to the bamboo arrangement (Fig. 4.6 and Fig. 4.7 a). The dynamic shape of the ferrofluidic plug is found to be similar for all applied field strengths with a downstream slope angle of $15^{\circ}$. As we have been discussed in the previous chapter, the fixed geometrical constriction with downstream slope angle $\lesssim 45^{\circ}$ could stabilize the metastable droplet arrangement more easily than the constriction with steep slope angle. Similarly, the ferrofluidic plug with less downstream slope angle can quench the droplet arrangement into metastable region.
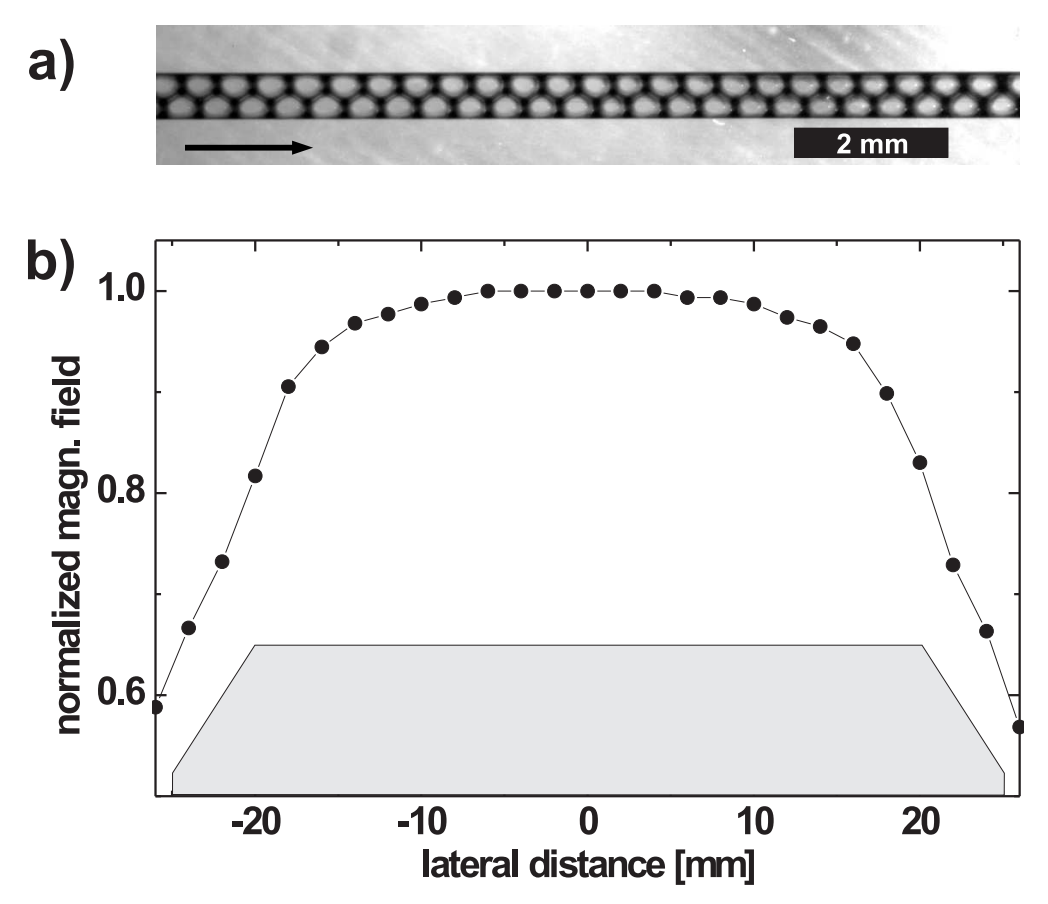

Figure 4.5: a) Optical images of a gel-emulsion with $\phi \approx 0.85$ exposed to a homogeneous magnetic field. The microfluidic channel is placed at the center of the pole shoe. A homogeneous magnetic field neither leads to the formation of a plug nor aligns the emulsion lamella parallel to the magnetic field up to the tested field strength of $1.5 \mathrm{~T}$. b) The relative magnetic field strength normalized to its maximum value is given as function of the lateral position (measured from the center of the pole shoe) for a wide pole shoe with $45 \mathrm{~mm}$ face. The shape of the pole face is sketched at the bottom of the graph in light grey. 
Fig. $4.7 \mathrm{~b}$ displays the normalized magnetic field strength as a function of the distance from the center of the rotationally symmetric pole shoe with $r=1.75 \mathrm{~mm}$. The magnetic field strength decreases from $100 \%$ at the center of the pole shoe to less than $20 \%$ at a distance $\mathrm{r} \geq 20 \mathrm{~mm}$. The shape of the pointed pole shoe is sketched at the bottom of the graph in Fig. 4.7 in dark grey. For both pole shoes, the magnetic field does not change qualitatively if we vary the magnetic field strength produced by the electromagnet.

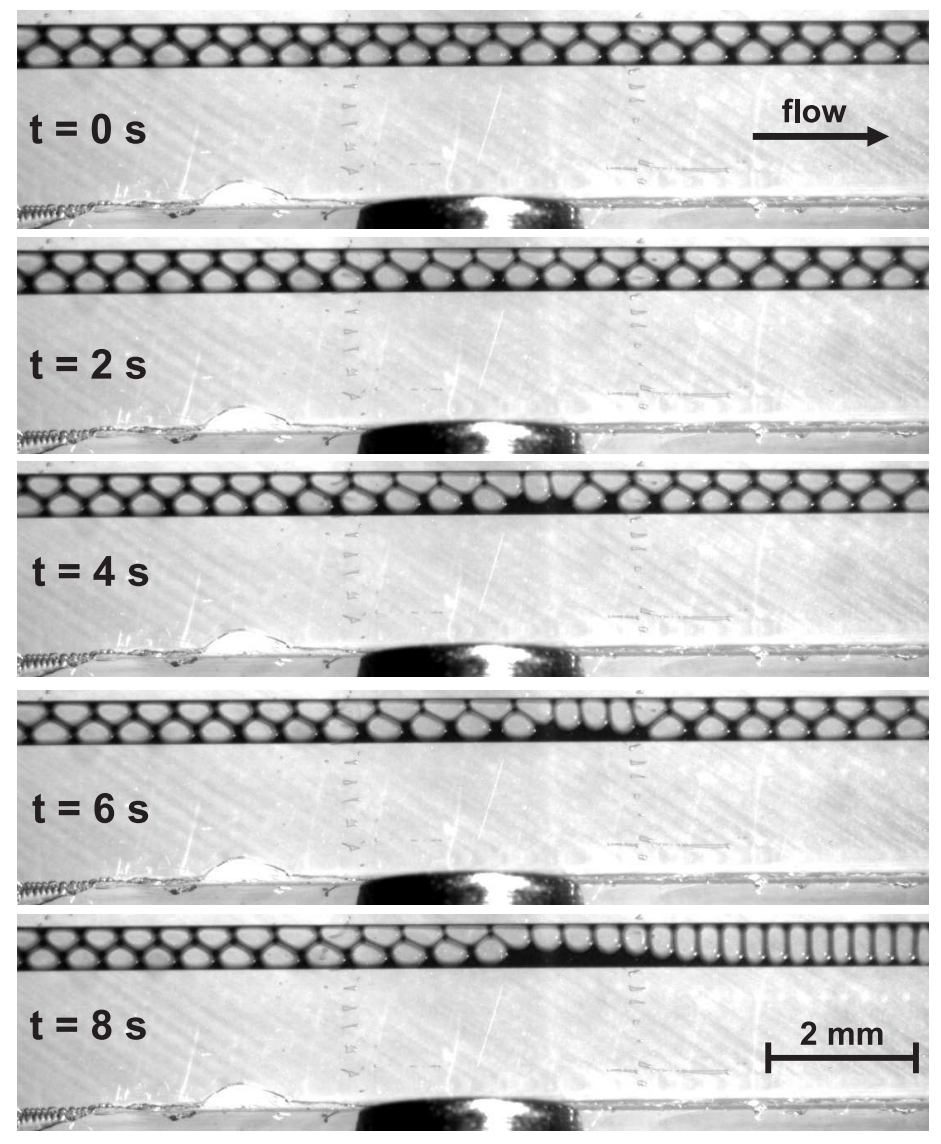

Figure 4.6: Time series of the development of ferrofluidic plug. At time $t=0$ the magnetic field is switched on to effect a transition from a zigzag to a bamboo structure. The peak value of the magnetic field strength was $280 \mathrm{mT}$ at the center of the pole shoe.

In case of a flowing emulsion, the hydrodynamic drag force deforms the shape of the plug and shifts it down stream relative to the symmetric distribution of the magnetic field, as shown 

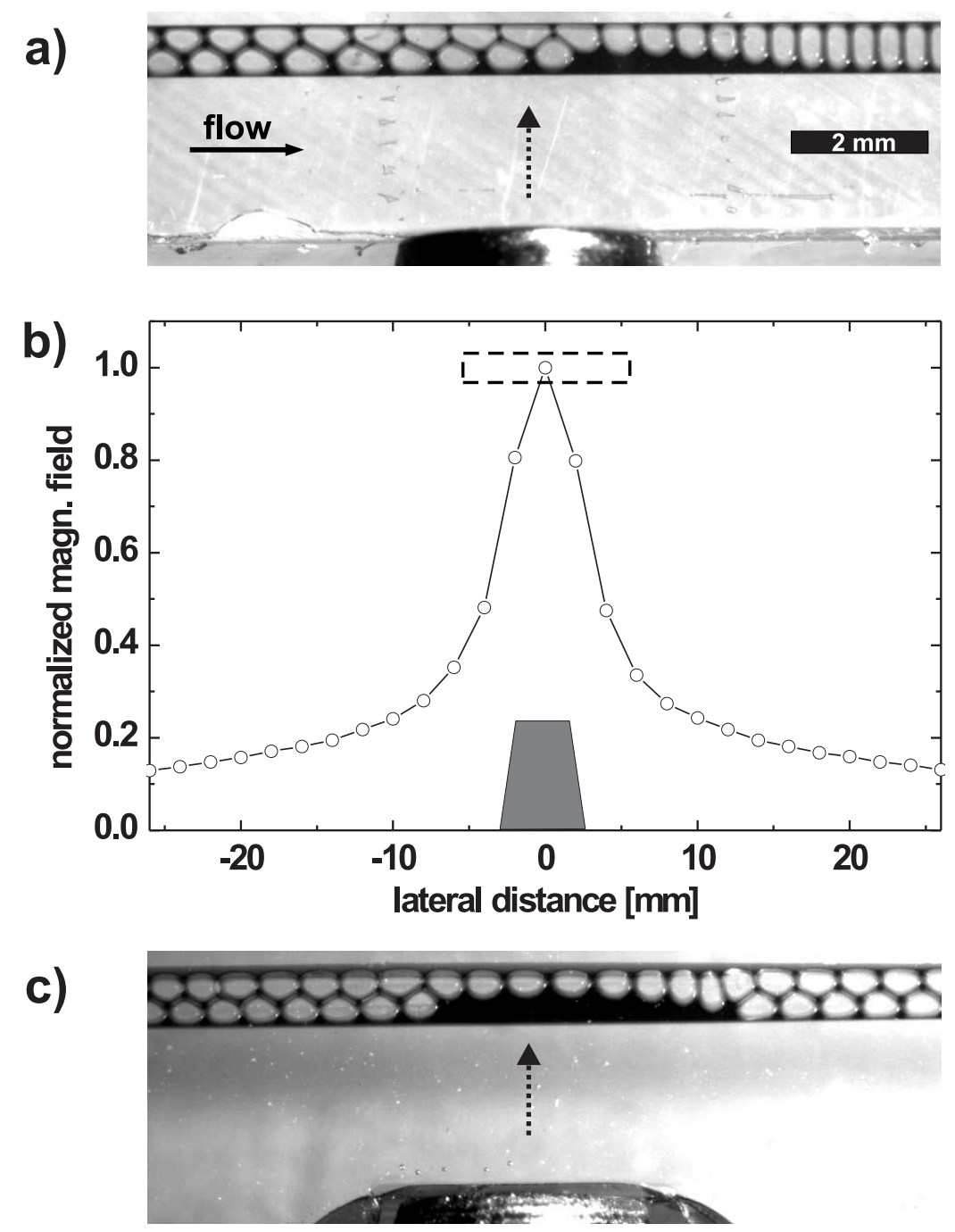

Figure 4.7: a) Optical micrograph showing a switching between a zigzag and a bamboo structure by inhomogeneous magnetic field in case ferrofluid used as a continuous phase. The center of the respective pole shoe is indicated as dashed arrow (magnetic field strength at the center of the pole shoe is about $200 \mathrm{mT}$ ) b) The relative magnetic field strength normalized to its maximum value is given as function of the lateral position (measured from the center of the pole shoe) for a pointed pole shoe. The shape of the pointed pole shoe is sketched at the bottom of the graph in dark grey. Dashed lines denote area of the micrographs (a) and (c). c) Ferrofluid plug formation at a quasi-static droplet arrangement. The ferrofluidic plug forms at the position of highest magnetic field strength, that is given by the geometry of the pole shoe. In case of flow in the microfluidic channel, the plug is shifted downstream due to the hydrodynamic drag force, depending on the flow velocity. The height of the plug, however is given by the absolute value of the magnetic field strength. 
in Fig. 4.7 a. In this case the applied flow rate is considered to be medium, the production rate is about 10 drops per second. For a static droplet arrangement which does not move along the channel, a plug forms which is symmetric with respect to the center of the pole shoe, see Fig. 4.7 c. However, here the system is not completely static but the flow rate is very low compared to the dynamic image shown in Fig. 4.7 a.

By varying the magnetic field strength, we can continuously vary the local channel width due to the ferromagnetic plug. Using this possibility, the critical channel width $\mathrm{W}_{c}$ to induce switching between a zigzag and a bamboo arrangement can be precisely determined for a given drop size and the volume fraction. In Fig. 4.8 the critical channel width normalized by the drop size, $\mathrm{W}_{c} / \sqrt{A}$ which is needed to induce a transition from a zigzag to a bamboo arrangement is plotted as a function of the dispersed volume fraction. For a channel width larger than the experimental values $\left(\mathrm{W}>\mathrm{W}_{c}\right)$, the zigzag arrangement persists. For smaller "effective" channel widths $\left(\mathrm{W}<\mathrm{W}_{c}\right)$, the zigzag arrangement is forced into a bamboo arrangement.

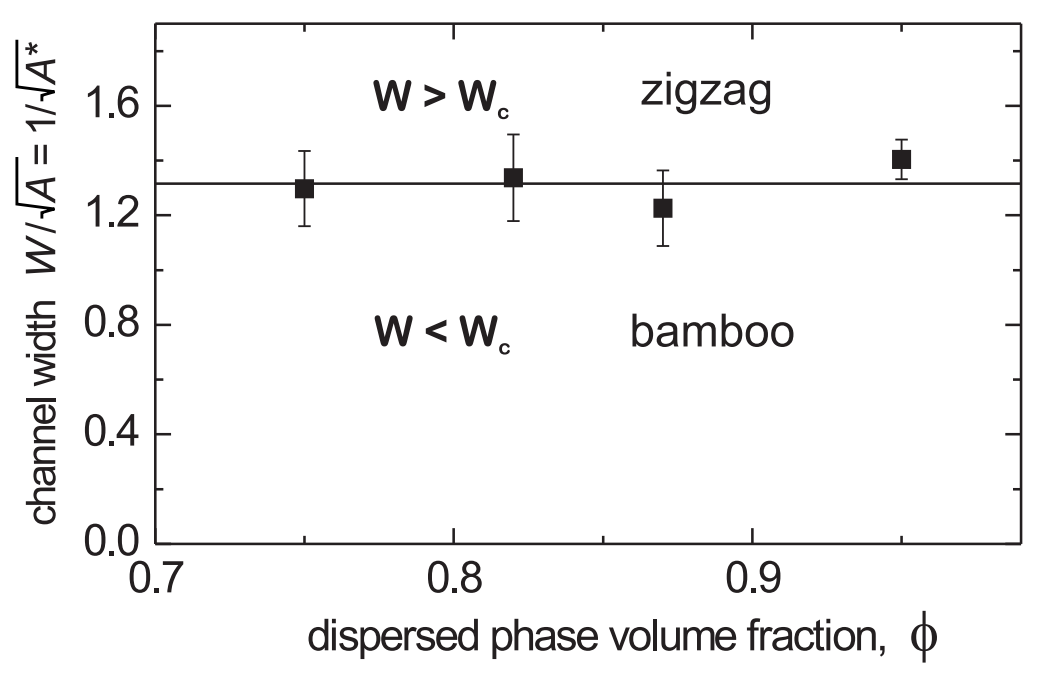

Figure 4.8: The normalized critical "effective" channel width $W / \sqrt{A}=1 / \sqrt{A^{*}}$ required to induce the zigzag to bamboo transition plotted as function of the dispersed phase volume fraction $\left(A^{*}=0.42\right)$. The local channel width $\mathrm{W}$ is varied by the constriction height generated by the ferromagnetic plug. The horizontal line at $1 / \sqrt{A^{*}}=1.32$ corresponds to the point of the transition between zigzag and the bamboo arrangement as obtained from the $2 \mathrm{D}$ geometrical foam model. 
According to the geometrical 2D-model given in the chapter 3, the zigzag arrangement cannot exist for channel widths smaller than $W / \sqrt{A}=1 / \sqrt{A^{*}} \approx 1.32$, which therefore sets an upper bound for the existence of the bamboo arrangement. This constraint is found to agree very well with our experimental results. Interestingly, the critical "effective" channel width is independent of the volume fraction of the dispersed phase and purely given by the size of the droplets with respect to the channel width. Experiments with a geometrical constriction micromachined into the microfluidic channel revealed a critical channel width of $W_{c} \approx 1.4 \pm 0.2$ at a dispersed phase volume fraction of $\phi=0.85$ in agreement with both the ferrofluid data and the theoretical expectation, shown in Fig. 4.8. Therefore, similar to the geometrical channel constrictions, the bamboo arrangement can be driven into the metastable region creating a temporally variable constriction forming a ferrofluidic plug. When channel width increases to it's original width after the ferrofluidic plug, either the bamboo arrangement could maintain as a metastable arrangement or could transform back to the zigzag arrangement, depending on the downstream slope angle and the droplet size. The larger the drop size associated with the less downstream slope angle of the ferrofluid plug can stabilize the bamboo arrangement easier after the plug.

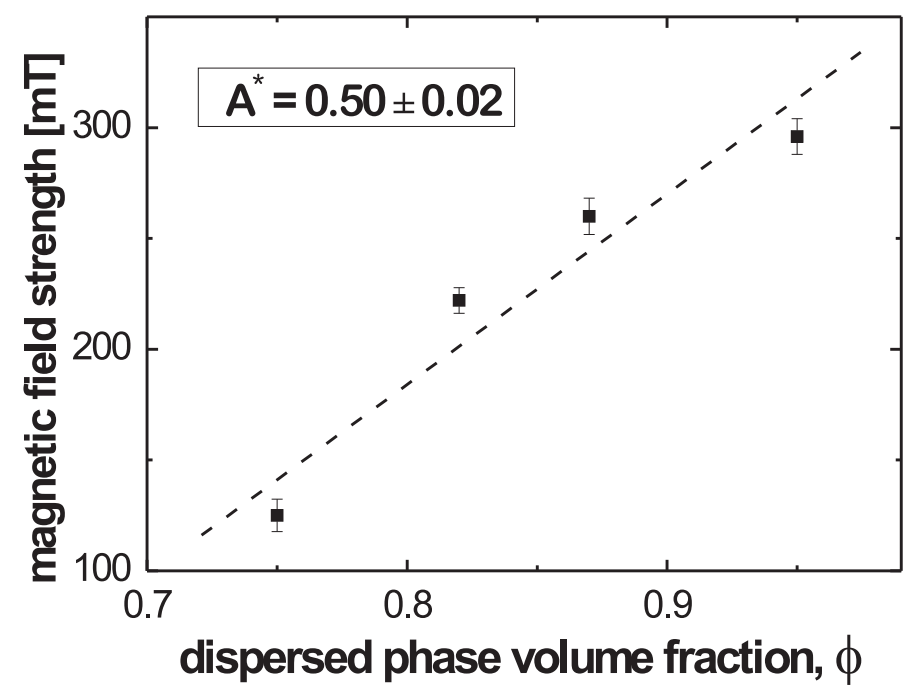

Figure 4.9: Magnetic field strength needed to form the critical plug height as function of the dispersed phase volume fraction. Higher dispersed phase volume fraction requires higher magnetic field strength to effect a transition. The dashed line is a guide to the eye. 
The minimum magnetic field strength needed to induce a transition from a zigzag to a bamboo arrangement i.e. in the narrow region where ferrofluid plug accumulated strongly varies with the continuous phase volume fraction, cf. Fig. 4.9. This is a consequence of the increased (negative) Laplace pressure in the Plateau borders for reduced continuous phase volume fractions [128, 129]. As the ferrofluid plug must grow by drawing liquid from the thin lamellae and Plateau borders, the plug formation is opposed to the dispersed phase volume fraction. Thus, the higher the dispersed phase volume fraction the larger the magnetic field strength is required to induce the transition.

Our findings indicate that the same switching effect induced by geometric constriction can be achieved using a ferrofluid. If a laterally inhomogeneous magnetic field is applied, ferrofluids tend to flow toward regions of increasing magnetic field strength, due to their high magnetic susceptibility. As a consequence, the ferrofluid (continuous phase) forming the lamellae of the gel emulsion should accumulate at the point of highest magnetic field strength. Thus, the volume fraction of the dispersed phase varies laterally along the channel in the vicinity of the pole shoe. The locally accumulated ferrofluid forms a plug at the center of the pole shoe, which acts as a geometric constriction just as demonstrated in the previous chapter. However, in this case the switch is active, allowing for on-demand manipulation of droplets. For the ferrofluid emulsions this is achieved by adjusting the current passing through the electromagnet.

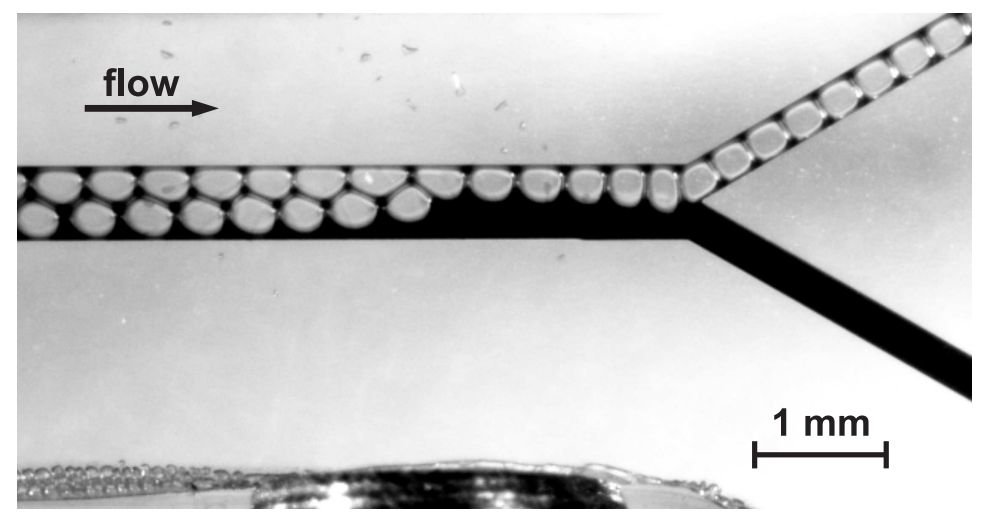

Figure 4.10: The ferrofluidic plug can act as a valve when used to obstruct one of the branches of a Y-junction.

There are two possible applications for microfluidic processing where the manipulation of droplet arrangements could be used. First, we consider a simple Y-junction. It can either act as a 'zipper', separating or combining two droplet rows reversibly (Fig. 4.1 a), or it can split 
the droplets when they arrive as a bamboo arrangement (Fig. 4.1 b) [105, 112, 115]. Thus, by changing the arrangement of the droplets (bamboo or zigzag) we can alter the operation of the Y-junction. Furthermore, we employed our magnetically responsive water-in-ferrofluid system in the context of a branched microchannel, cf. Fig. 4.10. Carefully positioning the electromagnet with respect to the junction of a Y-piece, we can accumulate the ferrofluid in front of one channel of the Y-junction. In this case, the ferrofluid will fully or partially obstruct one of the channel branches, behaving as an externally actuated valve. As one expects from our results in straight channels, the field strength needed to form a plug large enough to block one branch of the Y-junction depends on the dispersed phase volume fraction. 


\section{Chapter 5}

\section{D Structural Transitions of a Static Droplet Arrangements}

As we discussed in the previous chapters ( 3 and 4), the stability of certain droplet arrangements depends on the drop size with respect to the channel dimension. As we have seen in the experimental results so far, the dispersed phase volume fraction has an influence on the droplet arrangement. Fig. 5.1 shows a paradigmatic example of how a transition between metastable arrangements can be induced by changing the dispersed phase volume fraction. Here a zigzag structure is "forced" to transform into a bamboo-like structure by adding the continuous phase to the main channel via three side channels (upper left), thereby decreasing the dispersed phase volume fraction of the emulsion. The normalized $2 \mathrm{D}$ area of the droplet is $A^{*}=0.52$ in this example, which is close to the coexistence between bamboo and zigzag arrangements as derived from minimizing the surface free energy in 2D (as demonstrated in chapter 3), therefore transformations between those arrangements can be easily achieved. Furthermore, a meta-stable bamboo-like structure is transformed back to a zigzag structure by increasing the dispersed volume fraction again via withdrawing the continuous phase from the main channel by three of side channels (lower right). However, in this example, the emulsion was flowing continuously in the microchannel. In the following section we will discuss the same structure transition as a function of the dispersed phase volume fraction in the static case where certain droplet arrangements are in their equilibrium configuration.

To study the influence of the dispersed phase volume fraction as an extensive parameter on the stability of certain droplet arrangements we apply a lateral force to the droplets along the length of the channel. The preliminary results we have achieved so far show good agreement 
with a numerical calculation of the two-dimensional model of emulsion arrangements.

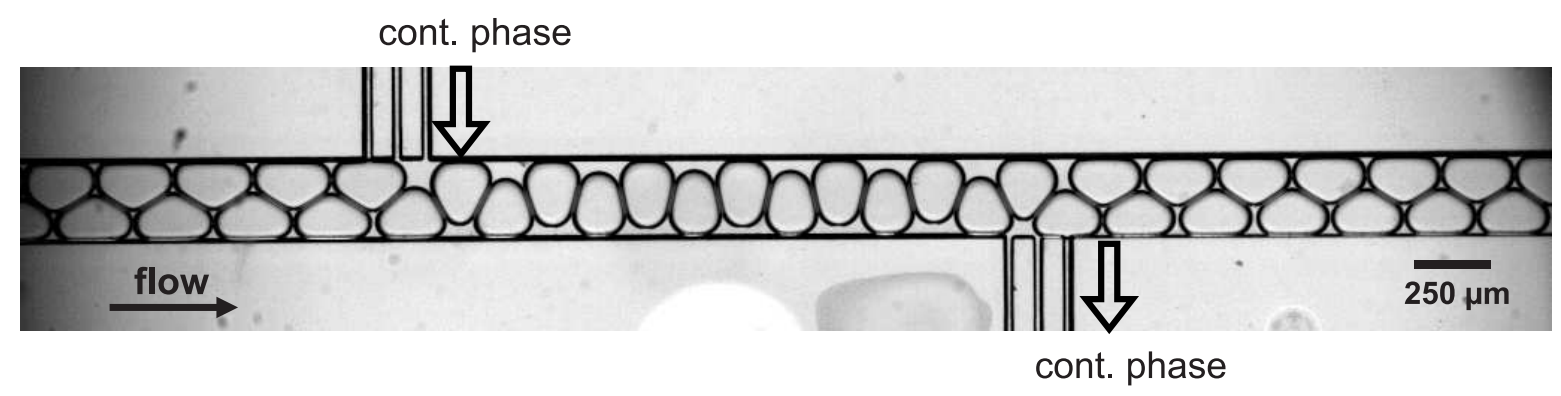

Figure 5.1: Transitions from zigzag to bamboo and the bamboo to zigzag arrangement are possible by changing the volume fraction of the emulsion. The flow direction is from left to right as indicated by the solid arrow. The open arrows at the side channel indicate the flow direction of the continuous phase.

\subsection{Device design and experimental conditions}

To achieve the static droplet arrangements in our microfluidic channel system, first, we had to optimize our channel design and second, the emulsion flow needed to be stopped without disturbing the desired droplet arrangement. Once the system was equilibrated a defined lateral force was applied to the droplets using a plug composed of an oil which is neither miscible with the continuous phase nor the dispersed phase of our emulsion (e.g. perfluorinated oil). The device fabrication started with creating a design in a CAD program. The microfluidic device used in our experiments consisted of 7 or 8 inlets and outlets (number of inlets and outlets depend on particular design of the microchannels) which was fabricated using photolithography of SU8 photoresist cf. Fig. 5.2 (for detailed description see chapter 2, microfluidic device design and fabrication section). The microfluidic device has two outlets (see Fig. 5.3) which are connected to a T-valve, so that regulating the T-valve one can open the first outlet while the other outlet is closed. To monitor the pressure, we used a pressure sensor that was connected to the channel via a tubing system. In fact, the pressure sensor measured the pressure difference between the plug channel and the side channel while the perflourinated oil was pushed through the channel. The flow rates were adjusted using microfluidic syringe pumps. Typical flow rates used in this experiment were $<10 \mu \mathrm{l} / \mathrm{h}$, which was achieved by using syringes (Hamilton) with volume of 50-100 $\mu$ l. 
At the beginning of an experiment the valve for outlet 1 is closed and all the produced droplets were flowing in direction of outlet 2 (cf. Fig. 5.3 ). When the channel is filled with droplets we close outlet 2 and open outlet 1 to direct all new generated droplets to outlet 1 . Subsequently, the flows of the continuous and the dispersed phases are stopped. It takes a few seconds until the flow stops and several minutes until the system gets to complete rest due to the pressure build up in the system. The inner diameter of the teflon tubing was chosen to be as small as possible (cf. Fig. 5.2) in order to keep the liquid volume smaller so that the flow could be stopped immediately without causing too much disorder in the channel. In our experiments we used teflon tubing with a $0.2 \mathrm{~mm}$ inner diameter. Now the experimental area (denoted area in the image of Fig. 5.3 ) is filled with monodisperse droplets with a volume fraction of $\approx 60 \%$ so that the droplets just touch each other. Once the system has equilibrated, small valves attached to the tubing of two "plug channels" 1, 2 are opened gently. After that, the plugs were driven forward stepwise by syringe pumps. The excess continuous phase is drained via the small side channels and the droplets are compressed. During this compression of the droplets the process is recorded by continuously capturing optical micrographs and monitoring the pressure between the side channel and the plug channel cf. Fig. 5.4. The pressure difference for a given droplet arrangement was taken when the new arrangement is equilibrated and the pressure difference had reached a stable value.

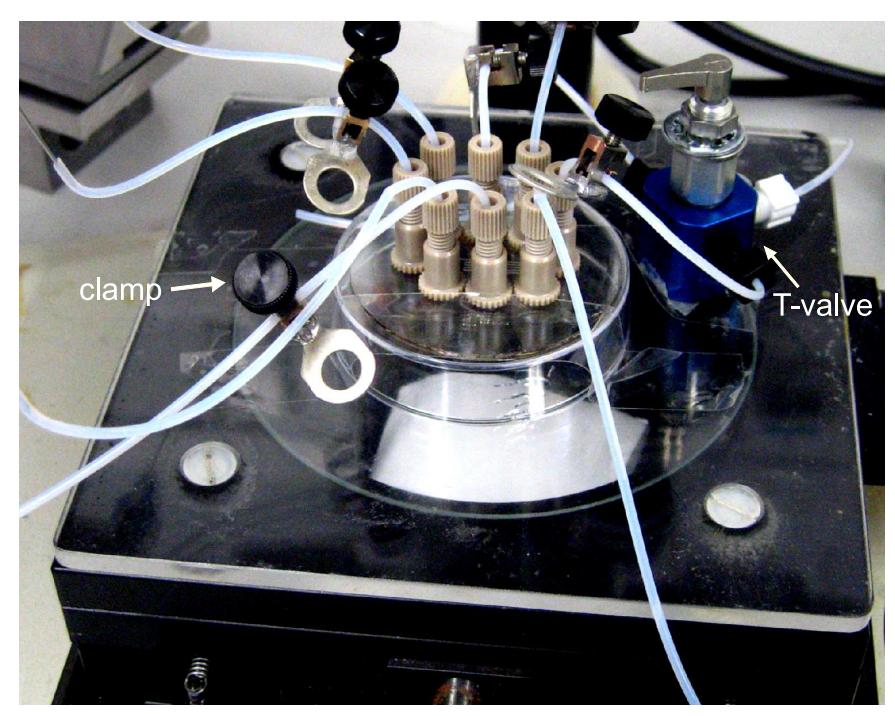

Figure 5.2: Experimental set-up with the microfluidic chip, t-valve and small clamps used in the experiment. 


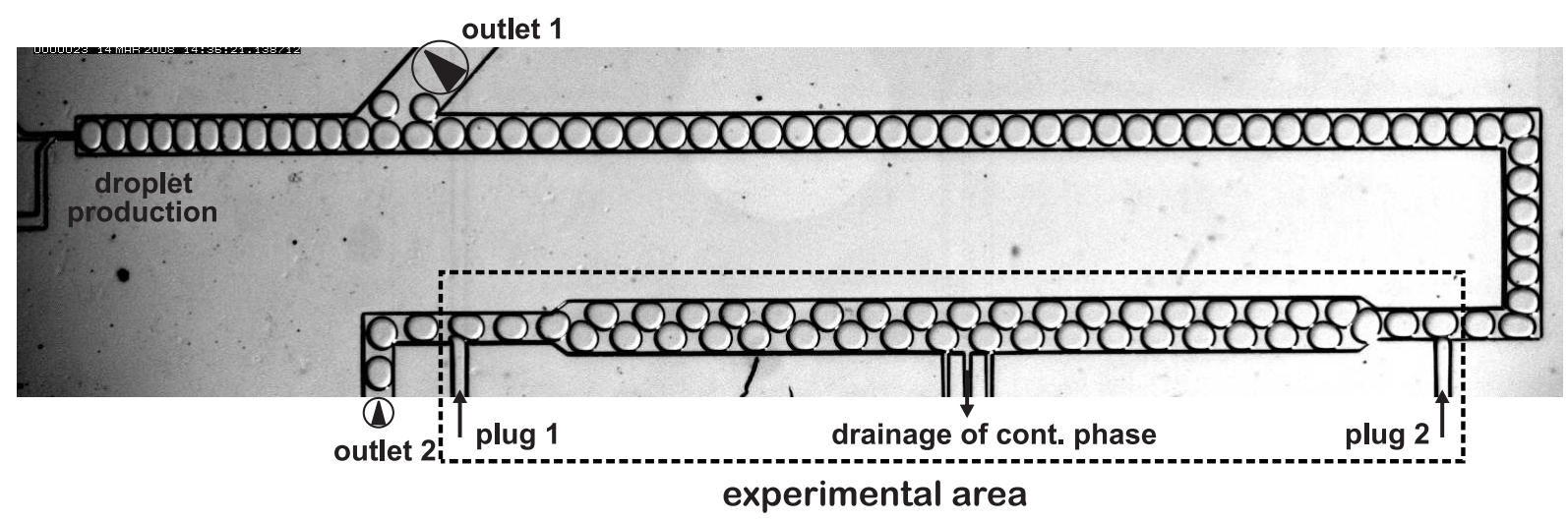

Figure 5.3: A micrograph of our channel network showing the production part, plug channels, side channels, and two outlet channels. Dashed lines denote the experimental area of interest.

\subsection{Results and discussion}

In our experimental system, the lateral force which is applied to the emulsion arrangement is the accessible control parameter. As a consequence of applying a lateral force the dispersed volume fraction changes and thus different droplet arrangements can be observed. Another control parameter that we have taken into account is the droplet size. Let us first discuss what observed in our static emulsion arrangements when the lateral force was applied for various rescaled drop sizes. In Fig. 5.4 where an initial configuration of a loose zigzag arrangement (see Fig. 5.4 a) with normalized drop size of $A^{*}=0.43$ was transformed into a close packed zigzag arrangement (see Fig. 5.4b) due to the applied lateral force. As a consequence of the presence of a lateral force in a direction of the channel, the droplets were compressed and the volume fraction of the emulsion was increased (the excess continuous phase escaped through the small side channels), thus we obtained different droplet arrangements. Increasing the lateral force even further did not change the droplet arrangement. Only the volume fraction of the dispersed phase had increased (Fig. $5.4 \mathrm{c}$ ). If repeat these experiments with a larger drop size $\left(A^{*}=0.58\right.$, cf. Fig. 5.5$)$ the initial one row arrangement is forced into a zigzag-like arrangement by applying a lateral force, cf. Fig. 5.5 b. Increasing the applied force result in the development of again a bamboo structure, as shown in Fig. 5.5 c. Increasing the lateral pressure further just changes the volume fraction whereas the bamboo arrangement is maintained (Fig. $5.5 \mathrm{~d}$ ).

If the droplets are smaller a zigzag arrangement is stable whereas for the larger drop size a bamboo arrangement is stable. However, there is a range between those two regions where 
(a)

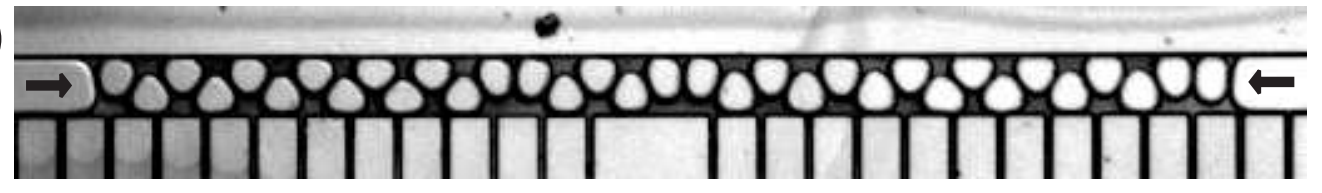

(b)

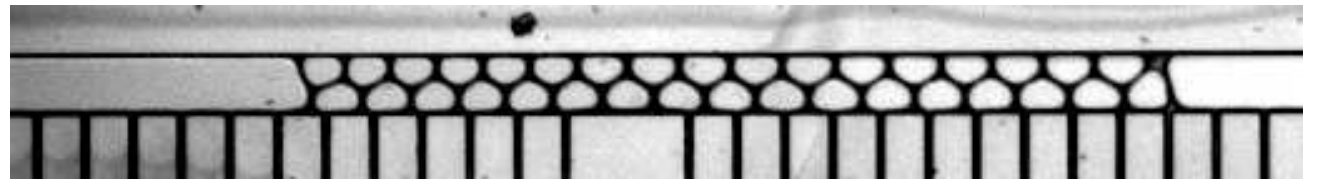

(c)

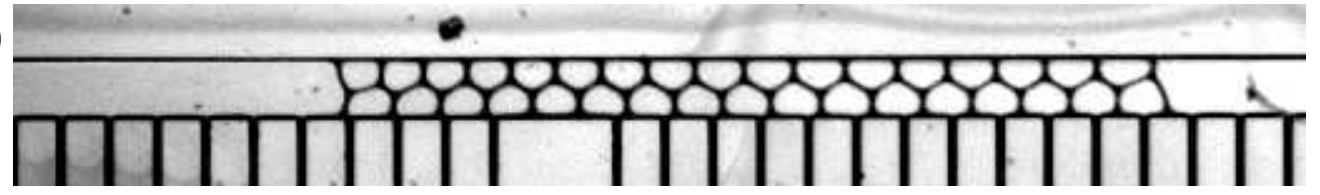

Figure 5.4: Time series of static emulsion arrangements, taken with different applied lateral forces, and different volume fractions. $A^{*}=0.43$.

a stable droplet arrangement will depend on an applied lateral force. The full experimental curves measured for the two drop sizes of $A^{*}=0.43$ and $A^{*}=0.58$ are shown in Fig. 5.6. For comparison, we have plotted our experimental curves together with the numerical results for the simple two-dimensional model for static emulsion arrangement, which was calculated by M. Brinkmann [130].

(a)

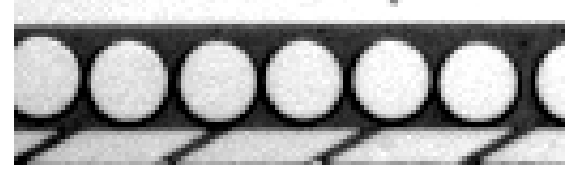

(b)

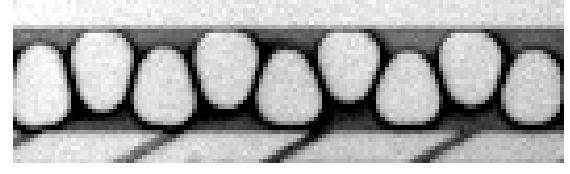

(c)

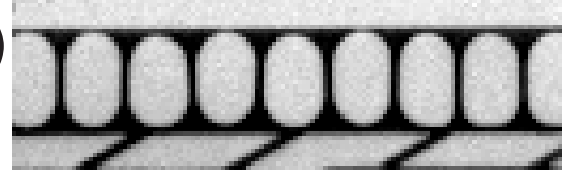

(d)

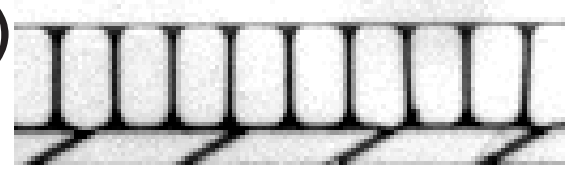

Figure 5.5: Time series of static emulsion arrangements, taken with different applied lateral forces, and different volume fractions. $A^{*}=0.58$.

In Fig. 5.6 the droplet arrangements as a function of a lateral force for various drop sizes are illustrated. Here, the dashed lines represent our preliminary experimental results and the solid lines are obtained by numerical minimization [130], using the "Surface Evolver". The transitions between a bamboo and a zigzag arrangement can occur if the applied lateral force is increased, cf. Fig. 5.6. 
The numerical results are obtained by minimizing the surface energy of a droplet arrangement at a given lateral force. Employing the final configuration of the previous minimization as the initial configuration of the following minimization one can increase or decrease the relevant control parameter by small steps after equilibrium has been reached. The step size of these changes has to be sufficiently small such that the actual droplet configuration stays close to the minimum of the interfacial energy. As shown in Fig. 5.6 in a certain range of the normalized drop size $A^{*}$, the lateral force into the direction of the channel may decrease with increasing volume fraction of the dispersed phase. This implies that a homogeneous distribution of droplets in the channel can be a subject to a mechanical instability as the droplets are compressed or decompressed, e.g. when the continuous phase is partially squeezed out or pushed into the emulsion. However, for a fixed amount of the continuous phase one may observe a spontaneous separation of an initially homogeneous arrangement into two homogeneous droplet arrangements with a higher, respectively lower volume fraction of the dispersed

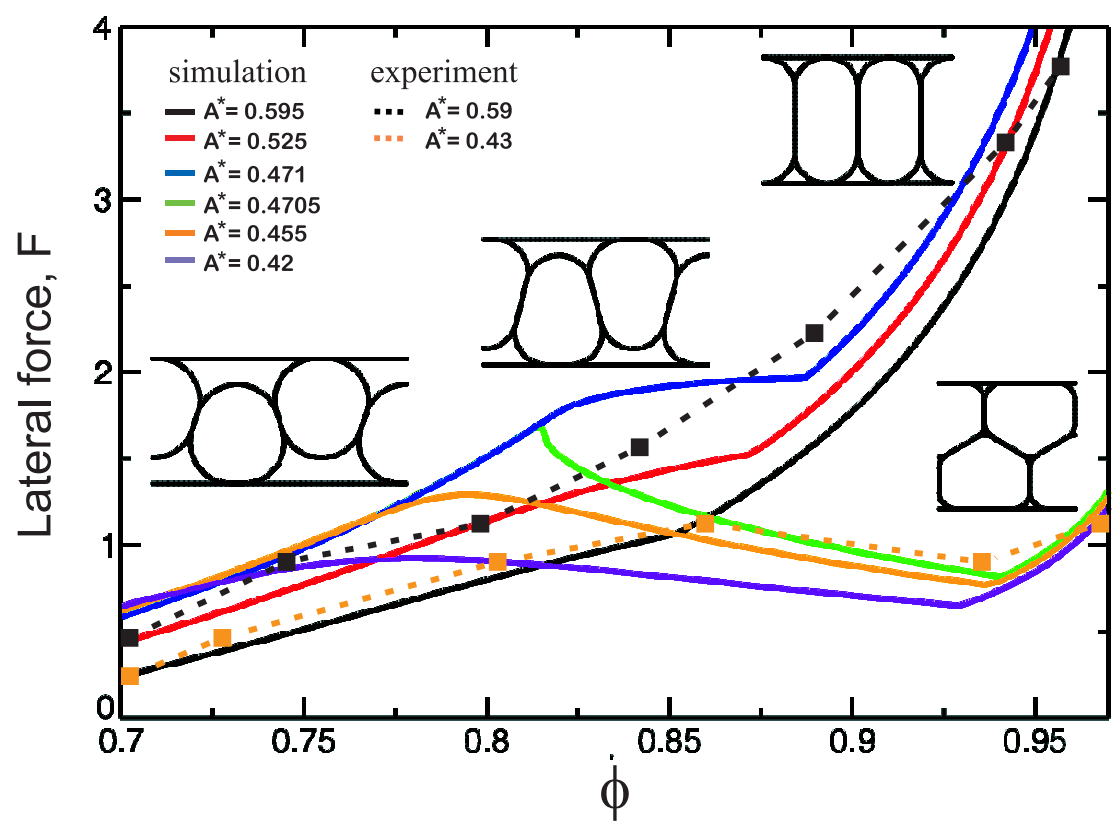

Figure 5.6: Lateral compressive force as a function of the volume fraction of the dispersed phase for different normalized '2D-drop volumes', $A^{*}=A / W^{2}$. The dashed lines are representing the experimental results, whereas the solid lines are obtained by simulation. The force shows a bifurcation to different equilibria at $A^{*}=0.4705$ and a volume fraction of the dispersed phase of 0.815 . 
phase. Qualitatively, the experimental results for the droplet compression agree well with the numerical calculation in that a monotone and a non-monotone dependence of force upon volume fraction can be obtained by varying droplet size, $A^{*}$ cf. Fig. 5.6. However, more detailed investigations need to be done in order to achieve quantitative agreement, especially to see the force bifurcation as is observed in the simulation. 



\section{Chapter 6}

\section{Formation and Manipulation of Droplet-Encapsulated Fibrin Network}

In the present chapter we use discrete microfluidics to study the encapsulated fibrin network formation and the properties. In order to investigate the evolution and manipulation of this robust network, we developed microfluidic devices that are capable of initiating fibrin network formation within droplets. This is achieved via targeted electrocoalescence between adjacent droplets and allows the early stages of the fibrin network to be observed directly. The use of droplets prohibits strong adhesion of fibrin to the microchannel walls during network formation by encapsulating the protein and enzyme within the dispersed phase. Furthermore, the incorporation of geometric structures into the microchannels enables the controlled deformation of individual droplets containing fibrin networks. The resulting droplets experience one of two conditions: either they traverse a pathway traveling continuously, or they park in a reaction chamber to fully evolve an isotropic network and then undergo controlled deformations.

\subsection{Fibrin}

The study of fibrin is motivated by the protein's biological relevance and ideal networkforming properties. Fibrin forms via the assembly of subunits of fibrinogen under conditions that dictate the physical properties of the network formed. Hemostasis, or healing of injured blood vessels via blood clotting, is initiated by the aggregation of platelets, which are small anuclear disks produced in bone marrow. Subsequent aggregation at the injury site is then aided by the presence of fibrin. Aggregation can also be detrimental in some cases, such as 
when foreign therapeutic scaffolds induce the formation of excessive blood clots [131]. The biodegradable nature and good tissue tolerance of fibrin networks have already been demonstrated in terms of commercially available wound covering agents. Furthermore, there is much interest in the deactivation of hemostasis with respect to artheriosclerosis [132], inflammationinduced coagulation [133], nervous system diseases such as multiple sclerosis [134], and certain cancers [135]. In addition, from a materials point of view single fibers of fibrin have been found to show remarkable elasticity[136]. Previous studies of fibrin formation have used bulk measurements, such as optical density or light scattering, to characterize fibrin gels [137139, 141]. Historically, most microscopy of fibrin networks in the literature resulted from electron microscopy measurements, which require staining and fixing of samples in processes that may alter the native structures [142-144]. While all of these previous studies have made important contributions to the understanding of fibrin formation, direct measurements of fibrin in its natural state are lacking.

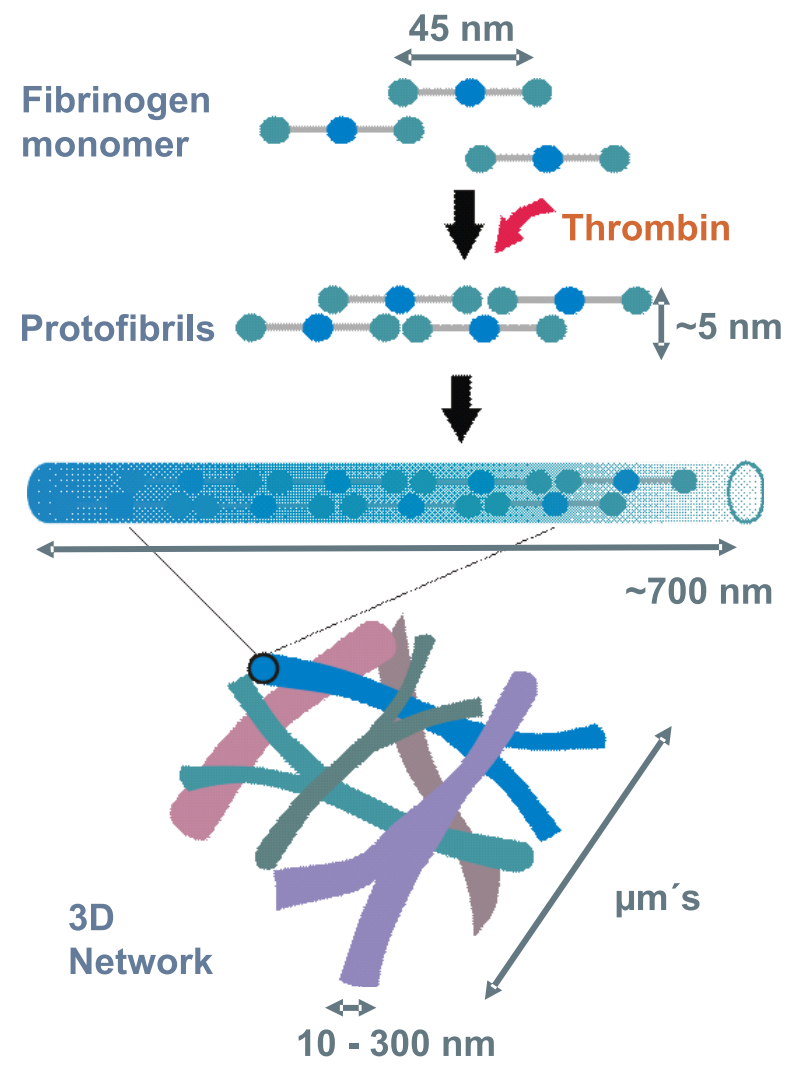

Figure 6.1: Hierarchical self-assembly of fibrin. 
The building blocks of fibrin are the fibrinogen monomers. They comprise about four percent of the total protein in blood plasma. Each fibrinogen molecule has a length of $45 \mathrm{~nm}$, and consists of a central domain flanked on either end by coiled-coil regions. Although the in vivo process of blood clotting involves a multi-step biological pathway, a minimal system may be conceived consisting only of the protein "monomers" of fibrinogen and the serine protease thrombin, cf. Fig. 6.1. A three-dimensional fibrin network is initially catalyzed by the presence of thrombin, which selectively cleaves fibrinopeptides located in the central domain of fibrinogen. This cleavage creates activated fibrinogen molecules that reassemble into protofibrils via knob-hole interactions. Subsequent aggregation of the fibrils into fibers eventually evolves into a three-dimensional network spanning micrometers, with properties that depend on salt and thrombin concentration [137-140].

\subsection{Experiments}

Microchannels were fabricated directly in SU8 photoresist using photolithography techniques. The typical channel dimensions are 20-120 $\mu \mathrm{m}$ wide and 20-50 $\mu \mathrm{m}$ deep. More detailed information about the device fabrication can be found in section 2.2.2.

Human fibrinogen (plasminogen, von Willebrand factor, and fibronectin depleted) was reconstituted in water to a concentration of $45 \mu \mathrm{M}$ according to the supplier's guidelines (Enzyme Research Laboratories, UK). Fluorescent fibrinogen Alexa Fluor ${ }^{\circledR} 546$ conjugate was also prepared according to guidelines, to a concentration of $4.4 \mu \mathrm{M}$ (Mobitec $\mathrm{GmbH})$. Fibrinogen and fluorescent fibrinogen were diluted 10:1 in a fibrinogen buffer (20 mM Sodium Citrate-HCl, pH 7.4). Human $\alpha$ - Thrombin, activity approximately $3000 \mathrm{NIH} \mathrm{u} / \mathrm{mg}$ (Enzyme Research Laboratories, UK), was reconstituted in water to a concentration of $94.6 \mu \mathrm{M}$ and further diluted in thrombin buffer (50 mM Sodium Citrate, 0.2 M NaCl, 0.1\% PEG - 10,000, $\mathrm{pH}$ 6.5). Relative molar ratios of fibrinogen to thrombin are given in the text as $[\mathrm{F}] /[\mathrm{T}]$. For comparative studies of beads in droplets, Fluoresbrite ${ }^{T M}$ carboxy NYO $0.50 \mu \mathrm{m}$ microspheres (Polysciences, Inc., Warrington PA) were diluted 1:1000 in thrombin solution and flowed through the microchip as usual.

Droplets of fibrinogen and thrombin solutions were formed in a continuous oil phase consisting of a low viscosity organic oil (Isopar ${ }^{T M} \mathrm{M}$, Exxon Mobil). In order to stabilize the emulsion, a non-ionic surfactant ( $2 \mathrm{wt} \% \mathrm{Span}^{\circledR} 80$, Sigma Aldrich) was added to the continuous phase. As shown in Fig. 6.2 the device contains two T-junction configurations where 
the droplets of the dispersed phase (in this case, protein solution) are formed [40, 41]. As the stream of the dispersed phase penetrates into the main channel, a droplet develops. The pressure gradient and shear force generated by the continuous phase distort the droplet in the downstream direction until the neck of the dispersed phase thins and breaks into a droplet (see Fig. 6.2 b). Droplets pair together at the following Y-intersection in the device and the two droplets pass through the electrodes very near to one another (Fig. 6.2 c). As shown in Fig. $6.2 \mathrm{~d}$ an applied potential $(50 \mathrm{~Hz})$ causes two neighboring droplets to coalesce due to an electric-field induced instability [36]. The details of electrocoalescence work has been discussed in section 2.5 .

\subsection{Fibrin network clotting in droplets}

In order to visualize the formation of fibrin, a microchip incorporating serpentine channels was designed. This allows the newly coalesced droplet, containing fibrinogen and thrombin, to fully evolve into a fibrin network while travelling over the extended length of the microchannel. The microscope remained in a fixed position on the device for these experiments. A high magnification objective (60X) was required to visualize the network in sufficient detail and, as a result, the camera field of view was only large enough to image one single droplet at a time. Imaging was carried out at a number of fixed positions along the serpentine microchannel to analyze the fibrin networks at various timepoints. Representative images are shown in Fig. 6.3 for $[\mathrm{F}] /[\mathrm{T}]=0.5$ and a droplet velocity $v=5 \mathrm{~mm} / \mathrm{s}$. The onset of mixing of the two solutions, seen in Fig. 6.3 a, is especially clear since the fibrinogen is fluorescent and the thrombin is not. Shortly after droplet coalescence, a homogeneous distribution of gray levels is measured within the droplet (Fig. 6.3 b). This confirms that complete mixing of the two components has occurred, and is set as the time point $t=0$ because we assume that the mixing is much faster than the fibrin-forming interactions between protein components. The evolution of fibrin formation and clotting is shown in Fig. $6.3 \mathrm{c}-\mathrm{f}$, which are taken at increasing distances (and, correspondingly, different reaction times $t$ ) along the device.

The presence of small, high intensity aggregates is found as the fibrin network develops. The number and size of these aggregates appear to be inversely related, and eventually a large, single clot of fibrin is found in droplets at the latest observation time, cf. Fig. $6.3 \mathrm{f}$. Moreover, the background intensity of the droplet evolves from light gray, at smallest $t$, to virtually black, at higher $t$. For a first analysis, the dimensionless parameter $\sigma^{*}$, defined here as the 

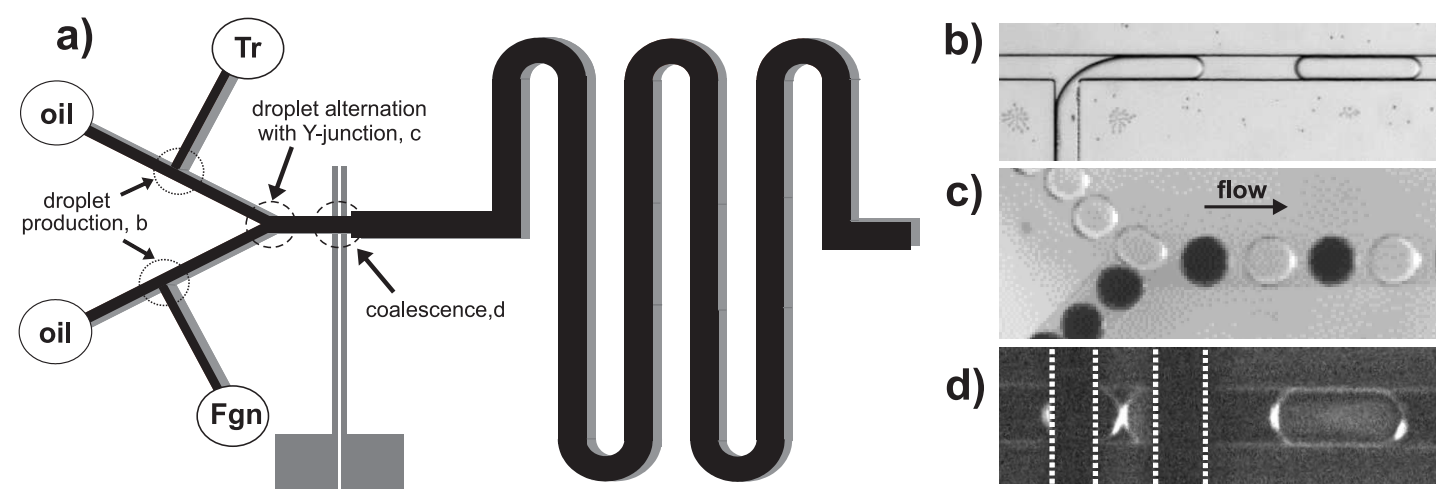

Figure 6.2: a) Microchannel design with 4 inlets. b) Droplets of thrombin (Tr) and fibrinogen (Fgn) solutions form at T-junctions. Droplets alternated b) at the Y-junction and d) coalesced when electric field is applied (electrodes marked with dashed lines).

normalized standard deviation of grayscale intensities in a droplet, was used to quantify these observations. This parameter is defined as $\sigma^{*}=(\sigma / I)_{t} /(\sigma / I)_{t=0}$, where I is the mean intensity and $\sigma$ its standard deviation.

The values of $\sigma^{*}$, averaged over many droplets, are plotted as a function of time in Fig. 6.3 . A substantial increase in $\sigma^{*}$ with increasing reaction time is found. This parameter seems to accurately describe the aggregation of fibrin as the droplets proceed through the device. Additionally, values of $\sigma^{*}$ for the same time point $(\mathrm{t}=2.7 \mathrm{~s})$ and droplet velocity $(\mathrm{v}=5 \mathrm{~mm} / \mathrm{s})$ but with different $[\mathrm{F}] /[\mathrm{T}]$ ratios $0.5,1$, and 5 are compared in the inset in Fig. 6.3. These ratios are changed by varying the upstream concentration of thrombin, [T]; in each experiment the concentration of fibrinogen, $[\mathrm{F}]$, is kept constant so that the amount of fluorophore is also conserved. It can be clearly observed that the aggregation is faster for the smaller ratio $[\mathrm{F}] /[\mathrm{T}]=0.5$, for which there is an excess of the enzyme thrombin compared to the substrate fibrinogen. Furthermore, for a higher ratio, $[\mathrm{F}] /[\mathrm{T}]=5$, the droplets remained homogeneously gray at all points in the microchip, indicating that the accessible time scale $\left(t_{\max } \sim 30 \mathrm{~s}\right)$ is not long enough in this case to visualize fibrin formation. The evolution of large scale aggregation, and the corresponding increase in $\sigma^{*}$, is particularly striking when compared to the values of $\sigma^{*}$ observed for fibrin networks formed under stopped flow conditions. In stopped flow conditions, $\sigma^{*}$ does not increase significantly from the starting value of 1 , when the two components have initially homogenously mixed. As seen in Fig. 6.3, the formation of a three-dimensional fibrin network in stopped flow does result in a slight increase in $\sigma^{*}$, but this value remains very low over all measured timescales (e.g. 1 hour, results not shown). 
Most importantly, $\sigma^{*}$ in stopped flow never approaches the higher values of $\sigma^{*}$ measured for moving droplets containing large aggregates.
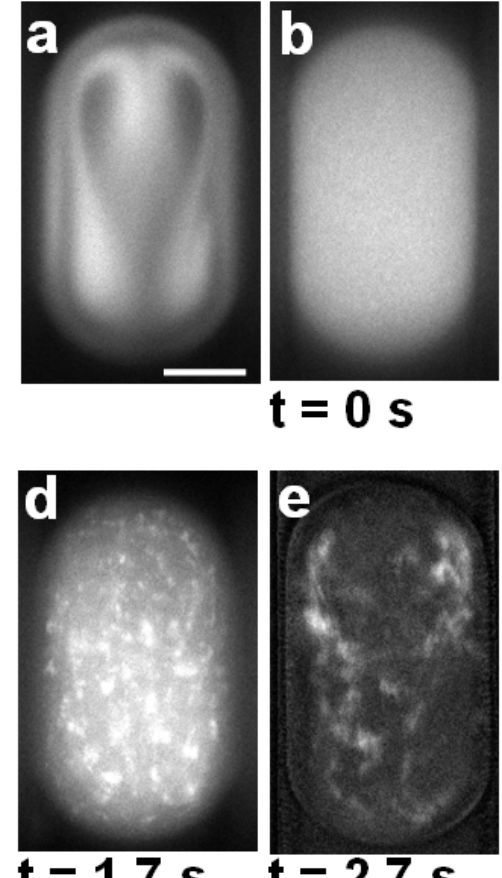

$\mathbf{t}=1.7 \mathrm{~s}$

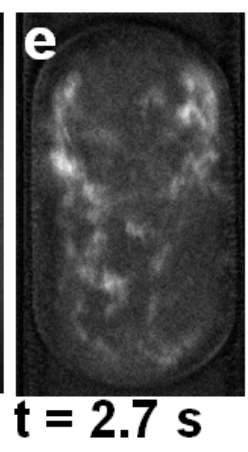

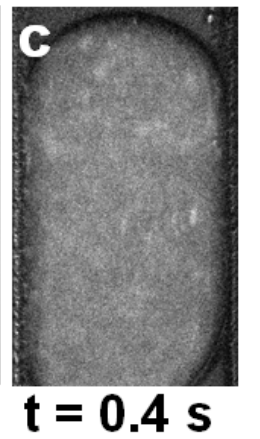

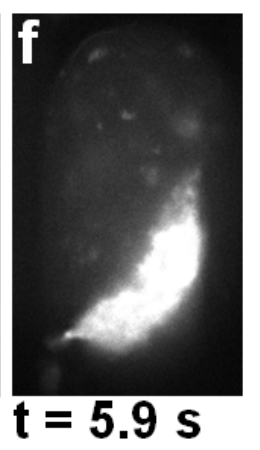

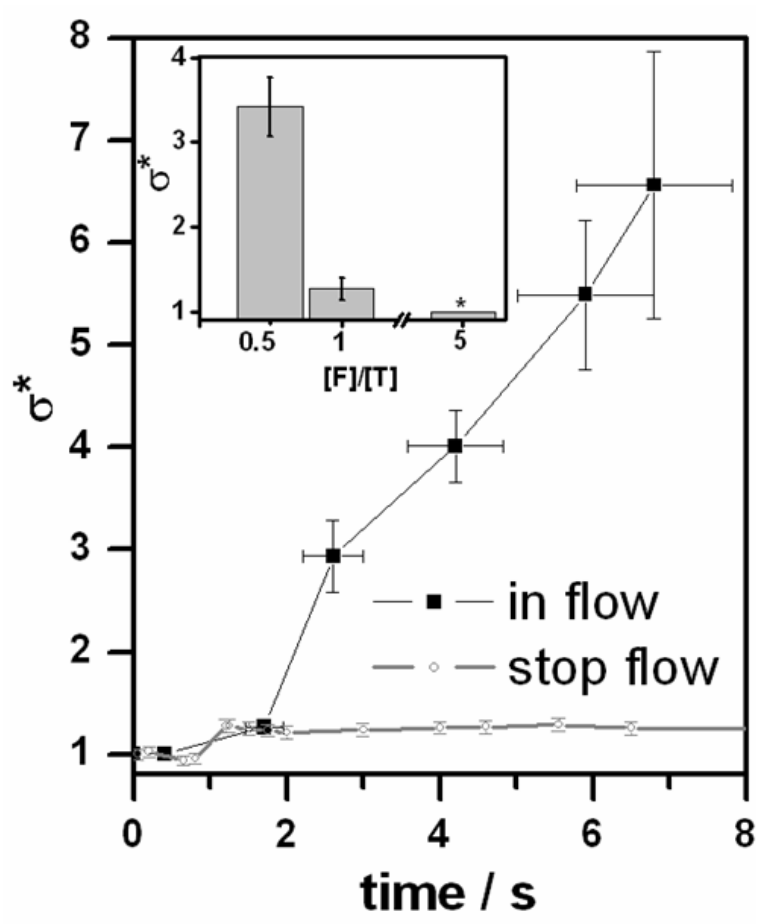

Figure 6.3: Fibrin forms in droplets $([\mathrm{F}] /[\mathrm{T}]=0.5, v=5 \mathrm{~mm} / \mathrm{s})$. Left: Representative images from (a) initial mixing stage to (f) final clotting; scale bar represents $60 \mu \mathrm{m}$. Right: Normalized standard deviation of droplet intensities $\sigma^{*}$ at different time points in flow compared to a droplet in stopped flow. Inset graph: For the same evolution time, $t=2.7 \mathrm{~s}, \sigma^{*}$ varies with $[\mathrm{F}] /[\mathrm{T}]$ and no fibrin formation is seen for high $[\mathrm{F}] /[\mathrm{T}]=5$ over the entire device (denoted with *).

The direct comparison between these two droplet conditions clearly demonstrates that the flow fields in moving droplets play an important role in the aggregation of fibrin networks. These flow fields apparently induce and accelerate fibrin interactions, culminating in protein aggregation. However, the circulating flow fields in microdroplets are known to be far from simple. Mixing between two liquids coalesced into a single compartment has been described as chaotic advection [145]. Recent measurements using confocal-PIV to follow the fluid motion in plug-shaped droplets indicate that indeed the circulating flow patterns are quite complex, with several vortices found at droplet edges [13, 14]. The flow direction and magnitude depends on the position within the droplet and, in similar plug-shaped droplets, the vortices 
at fluid-fluid interfaces can have velocities nearly $25 \%$ higher than the bulk droplet velocity (based on other measurements in our lab). In the case of fibrinogen and thrombin, there is a distinct consequence of this complex flow field which will now be addressed in more detail.

The formation of fibrin protofibrils is an intermediate step in the fibrin pathway, as illustrated in Fig. 6.1. The formation and lateral growth of these fibrils into larger fibers can, for the most part, be observed at optical resolution. As we cannot observe the smaller protofibrils using optical methods, the results presented here are indicative of larger fiber aggregation. The images taken at multiple timepoints clearly show the aggregation of protein into small clumps and then further aggregation into a single, large fibrin "particle". The irreversible aggregation is enhanced by local flow fields and is driven by physico-chemical intermolecular interactions of neighboring fibers with one another and with larger aggregates. The flow in the droplets increases particle-particle collisions, where particles can be fibrinogen, thrombin, fibrin fibrils or even fibrin aggregates. Thus, the flow field influences nearly all levels of protein formation and behavior. It also accelerates effects such as molecular crowding as well, which significantly alter the physical and chemical interactions of the protein, as qualitatively observed in the comparison with "stopped flow" conditions in Fig. 6.3. This local segregation between fibrin aggregates and solution appears to be a type of complex coacervation similar to what has been seen with other proteins [146]. Furthermore, the growth of these small particles into a large clump is likely to be mediated by electrostatic interactions. In summary, the impact of droplet flow fields on protein aggregation and clotting is an important consideration. The hydrodynamic effects within a droplet appear to significantly influence protein interactions and, therefore, comparisons between in vitro protein behavior and in vivo behavior must be carefully evaluated. However, it is plausible that flow fields in blood vessels may also promote molecular crowding and coacervation of fibrin in blood clots, both in blood flow as well as at the vessel injury site.

\subsection{Manipulation of fibrin network}

In order to reduce hydrodynamic influences on fibrin formation and to enable the online manipulation of fibrin networks, a second microfluidic device was employed. The ability to "park" a droplet creates an environment for internal droplet processes to proceed over long time scales. In this microchip, a reaction chamber allows the fibrin network to evolve in a quasi-stopped flow condition before it is mechanically manipulated through a series of mic- 


\section{Chapter 6. Formation and Manipulation of Droplet-Encapsulated Fibrin Network}

rochannel geometries. The droplet velocity is reduced in this reaction chamber by a substantial increase in the channel width, as shown in Fig. 6.4. The velocity of the droplets in the chamber is controlled not only by the inflow of liquid by the syringe pumps at the inlets but also by the withdrawal of continuous phase at an outlet from the side of the reaction chamber. Consequently, the droplet velocity is tunable and coalesced droplets can be held in the reaction chamber for a given residence time or "network ripening time", $t_{n}$.

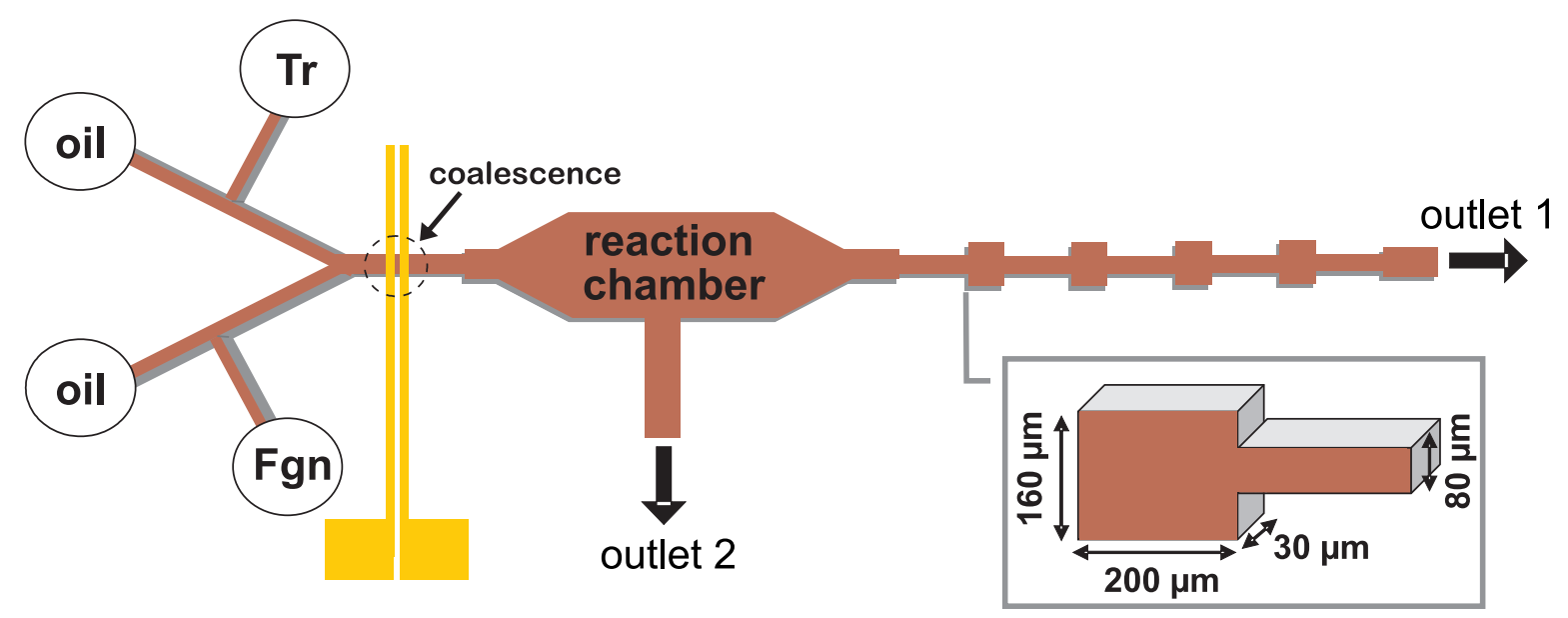

Figure 6.4: Microdevice incorporating a reaction chamber and geometric elements. The velocity $v_{2}$ of the side channel (outlet 2) controls the velocity of single droplets travelling through the microchannel outlet $1, v_{1}>v_{2}$.

Using this channel design, a ripening time $t_{n}$ on the order of tens of seconds in the reaction chamber is required to observe the type of network seen in Fig. $6.5 \mathrm{a}$ for $[\mathrm{F}] /[\mathrm{T}]=2$. At this time $t_{n}$, we observe an isotropic fibrous network that displays multiple crossing points of fibrils but does not show any large scale aggregation behaviors (i.e. those found under the flow conditions in the microchip described earlier). When the coalesced droplet sits in the reaction chamber, protofibril and larger fiber formation are able to proceed without any significant influence of hydrodynamic effects. Also, the fibers' thickness appears to be conserved for long observation times (e.g. 1 hour), which suggests that a steady state has been reached. The final result is a genuine droplet-spanning fibrin network. In the following analysis we treat this protein network as quasi-two-dimensional, due to the high aspect ratio of the droplet width to depth (5:1) and for simplicity of analysis. Some fluorophores are found at the dropletoil interface of coalesced droplets (possibly due to the surfactant system used here), but the fluorescence intensity at the interface does not change over long time periods and therefore 
this effect may be disregarded in a first approximation. Additionally, in every case where the fibrin networks form, some depletion of the network near the droplet-oil interface is observed, indicating that there are no specific interactions between the network and the droplet interface.
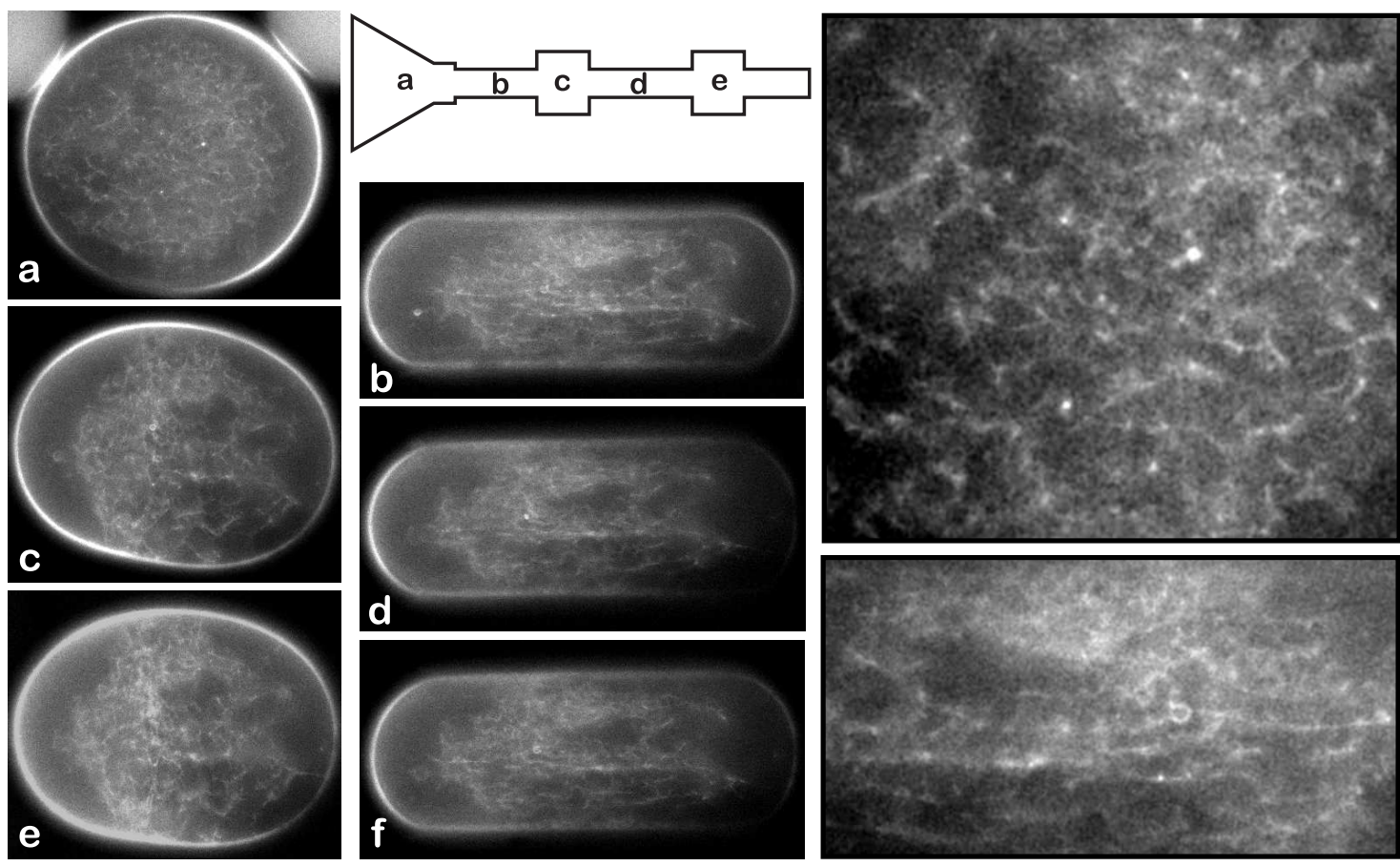

Figure 6.5: Images of the fibrin network in the same droplet $\left([\mathrm{F}] /[\mathrm{T}]=2, v_{\text {narrow }} \sim 200 \mu \mathrm{m} / \mathrm{s}\right)$ at positions a-e, corresponding to the sketch of device. Zoomed part from circle (right top) and deformed (right bottom) droplets are also shown. Scale bar is $40 \mu \mathrm{m}$.

The fibrous networks are manipulated as they pass through the microchannel, which contains several geometric obstacles. The microdevice shown in Fig. 6.4 contains narrow regions, which result in a compression of the droplets, followed by wide regions, which allow the droplet to regain its original circular shape. By appropriately adjusting the flow at the side channel of the reaction chamber, the droplets are driven through the final stages of the microdevice at velocities ranging up to $1 \mathrm{~mm} / \mathrm{s}$.

The images in Fig. 6.5 show one droplet prepared at $[\mathrm{F}] /[\mathrm{T}]=2$ as it passes through the microdevice. Due to the very slow flow speeds achievable using these devices, individual droplets could be followed by translating the microscope sample stage. Therefore, direct comparisons of the fibrin network during and after compression were possible. Comparing the squeezed networks in Fig. 6.5 a and Fig. 6.5 d, one sees qualitatively similar features. Centrally 


\section{Chapter 6. Formation and Manipulation of Droplet-Encapsulated Fibrin Network}

located fibers extend along the flow axis and a depletion region is found between the fibrin network and the surface of the droplet. The fibrin networks in the wide regions of Fig. $6.5 \mathrm{c}$ and Fig. $6.5 \mathrm{e}$, where the droplet shape reforms to a nearly circular cross-section, show a different fiber arrangement that approaches the isotropic network of Fig. 6.5 a. Similar features can be identified when comparing the circular droplets in Fig. $6.5 \mathrm{c}$ and Fig. $6.5 \mathrm{e}$, which indicates a restoration of general network features throughout the droplet's manipulation in the device. Altogether, Fig. 6.5 clearly shows that the fibrin networks formed under these conditions are able to compress and extend in a reproducible manner, indicating a considerable degree of structural integrity and network elasticity. Remarkably, this conservation occurs despite the fluid flow that percolates the fibrin network in the moving droplet.

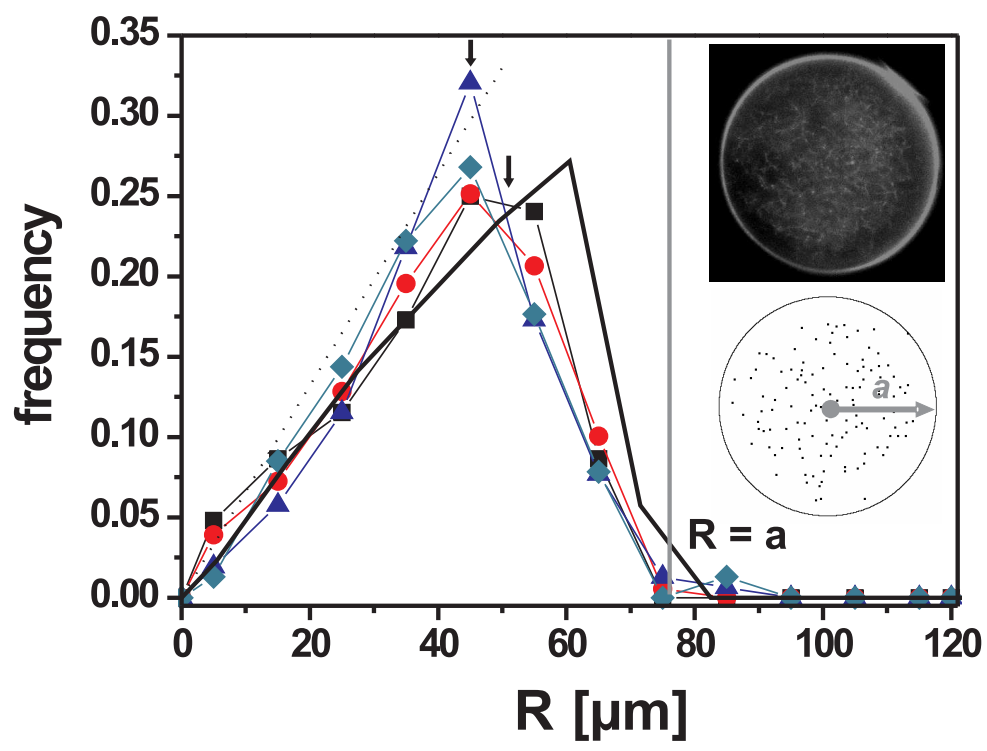

Figure 6.6: Histograms of network point distances $\mathrm{R}$ from center of mass of compressed droplets with $[\mathrm{F}] /[\mathrm{T}]=2$ and rate of deformation $\varepsilon=0.6 \mathrm{~s}^{-1}$. Network points (lower image) are determined from original data (upper image). Droplet radii and axis are marked with vertical gray line. Compared to the round droplet in the reaction chamber prior to any compression (black squares), circular droplets show a slight shift in peak frequency (arrows). The first, second, and third repetitions through the microchip are plotted with circles, triangles, and diamonds, respectively. For comparison, the histograms for beads in aqueous solution in a similar device are plotted in solid lines.

To appropriately describe the experimental condition of the droplets, we introduce a rate 
of deformation $\varepsilon=\Delta v / l$. Here, $\Delta v=v_{\text {narrow }}-v_{\text {wide }}$ is the velocity difference of the droplet in the two microchannel widths and $1=160 \mu \mathrm{m}$ is the typical length scale of deformation. To a good approximation, the velocities of the droplet depend on the width ratio of the deformation induced by the channels, $w_{\text {narrow }} / w_{\text {wide }}=80 \mu \mathrm{m} / 160 \mu \mathrm{m}=0.5$. This allows $\varepsilon$ to be calculated using only $v_{\text {narrow }}$, which was found to be consistently accessible experimentally, especially in cases where the fast moving droplet could not be tracked over long distances using the microscope stage. Using these assumptions, the rate of deformation can be calculated using $\varepsilon=v_{\text {narrow }} / 320 \mu \mathrm{m}$. Using these parameters, the data in Fig. 6.6 and Fig. 6.7 correspond to droplets undergoing a rate of deformation $\varepsilon=0.6 \mathrm{~s}^{-1}$.

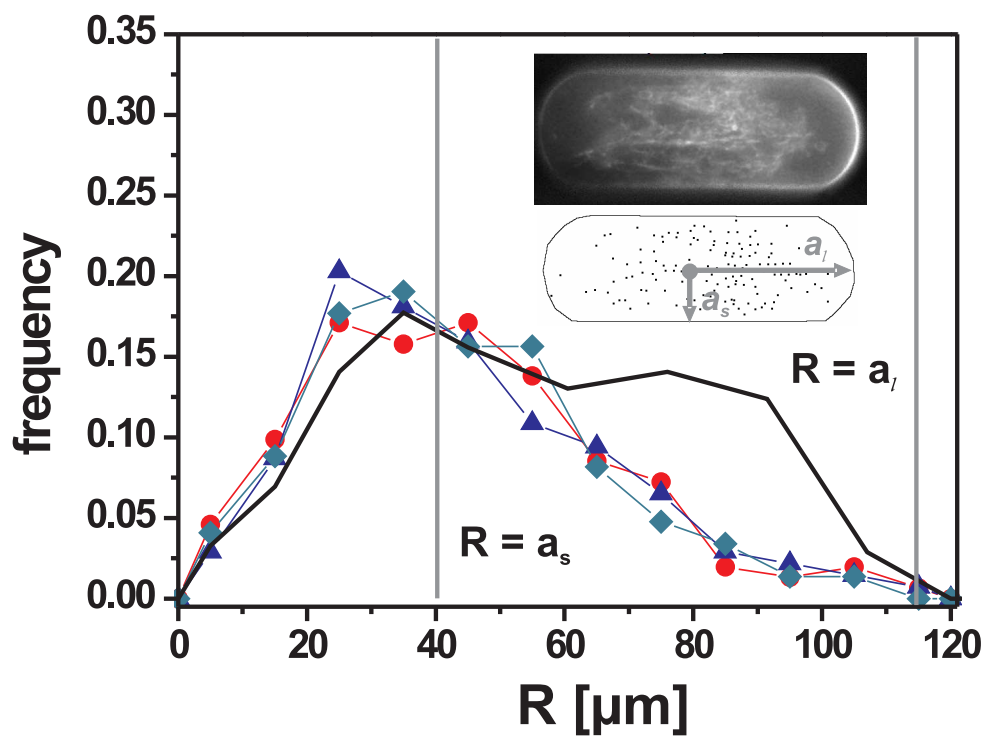

Figure 6.7: Histograms of network point distances $\mathrm{R}$ from center of mass of compressed droplets with $[\mathrm{F}] /[\mathrm{T}]=2$ and rate of deformation $\varepsilon=0.6 \mathrm{~s}^{-1}$. Network points (lower image) are determined from original data (upper image). Droplet radii and axes are marked with vertical gray lines. Compared to the round droplet in the reaction chamber prior to any compression (black squares), circular droplets show a slight shift in peak frequency (arrows). The first, second, and third repetitions through the microchip are plotted with circles, triangles, and diamonds, respectively. For comparison, the histograms for beads in aqueous solution in a similar device are plotted in solid lines.

Points of high intensity in the droplets are correlated to fibril cross-over in the interconnected fibrin network. An analysis of these points was conducted in order to quantify the structural similarities or differences between the networks in compressed or widened regions. 


\section{Chapter 6. Formation and Manipulation of Droplet-Encapsulated Fibrin Network}

Using image analysis software, the intense network features were identified by a binary algorithm with a conserved tolerance level. Examples of this process are shown in Fig. 6.6 and Fig. 6.7, where the original image frame is shown above the resulting point distributions for a droplet in both a wide (Fig. 6.6) and narrow (Fig. 6.7) channel. The positions of these features were used to calculate the distance $\mathrm{R}$ of each point from the droplet center (i.e. center of mass). Histograms of these distances are plotted in Fig. 6.6 and Fig. 6.7 for a single droplet passing through wide and narrow channel regions. For comparison, the distribution of network positions within the droplet in the reaction chamber, i.e. the native fibrin network, is also shown in Fig. 6.6.

All of the distributions shown in Fig. 6.6 and Fig. 6.7 for the circular droplets in wide regions approximately follow a linear scaling in $R$ up to a given threshold of around $\mathrm{R}=45 \mu \mathrm{m}$.

Depletion of the network from the droplet-oil interface is evident from visual inspection of these images and results in a decrease in the network point frequencies close to the physical droplet radius (gray line). These results agree with the expected distribution of points contained within a circular area, except for the depletion effect. The depletion is exacerbated by the presence of the interacting protein network. A comparison of the distribution of fluorescent beads in droplets studied in a similar device (black curve in Fig. 6.6 ) highlights the significant depletion that occurs with fibrin networks at the perimeter of the compartment. Furthermore, a larger depletion of the network is measured in the wide regions as compared to the native structure found in the reaction chamber (arrows, Fig. 6.6). This increased depletion is a consequence of droplet deformation and may also be due to neighboring fiber interactions driven by electrostatic and crowding effects. It is important to note that after the droplet initially experiences a deformation, the extent of depletion remains conserved. In fact, all of the distributions from wide channel regions shown in Fig. 6.6 (with the exception of the data from the droplet in the reaction chamber) overlay remarkably well. This suggests that the fibrin network recovers completely after repeated cycles of squeezing, which is qualitatively seen in the microscopy images.

A similar reproducibility is found when considering the distributions for the elongated droplets in the narrow channels, Fig. 6.7. In these regions the droplet assumes a plug of stadium shape, which is neither a rectangle nor an ellipse, and is described by a short axis $a_{s}$ and long axis $a_{l}$. These two length scales emerge in the distributions and are labeled with vertical gray lines in Fig. 6.7. For distances less than $a_{s}$, the distributions of intensities scale linearly until reaching a threshold distance of about $25 \mu \mathrm{m}$, and then decrease reflecting depletion from the walls at as in a manner similar to that found in the circular droplets. However, the linear 
scaling in the plugs has a slope that is higher than that measured in the droplets of Fig. 6.6 . This suggests an increased densification of the network when the droplet is compressed. The behavior of fibrin at distances between as and al significantly deviates from measurements of fluorescent beads in similar plugs (black line in Fig. 6.7). Although the distribution of beads remains high to distances of nearly $100 \mu \mathrm{m}$, the frequency of point distances in the fibrin network substantially decreases on the same length scale. However, the extent of depletion is conserved upon repeated compression of the droplet. This implies that the network behaves in an affine manner. Furthermore, there is no observation of delayed network recovery, because the distributions shown in Fig. 6.6 and Fig. 6.7 do not vary significantly at similarly shaped device positions.

\subsection{Coacervation of deformed fibrin networks}

At slow velocities and rates of deformation, $\varepsilon$, fibrin networks centered within the droplet can survive several cycles of compression. Furthermore, the network under these conditions is elastically deformable. However, at higher $\varepsilon$, non-affine deformations or inelastic network properties may be accessed if the deformation is faster than the recovery of the network. This is achieved by increasing the droplet velocity through the microchannel (of the microdevice shown in Fig. 6.4). At sufficiently high $\varepsilon$, two distinct phases are present within the droplet. This is clearly visualized in the upper image in Fig. 6.8, which shows a droplet with $\varepsilon=1.125$ $\mathrm{s}^{-1}\left(v_{\text {narrow }}=360 \mu \mathrm{m} / \mathrm{s}\right)$. The fibrin network concentrates toward the rear of the droplet with respect to the flow direction. For comparison, Fig. 6.8 also contains an image showing the centered network in a droplet $\varepsilon=0.5 \mathrm{~s}^{-1}$. Based on observation of droplets moving at different velocities, the phenomenon of extreme fibrin separation is found for droplets experiencing a rate of deformation $\varepsilon>1 \mathrm{~s}^{-1}$.

A comparison of droplets at a range of velocities is plotted in Fig. 6.8 for $[\mathrm{F}] /[\mathrm{T}]=2$. Images can once again be analyzed using the standard deviation of intensities normalized by the mean intensity. In this case, it is appropriate to use the relation $\sigma=(\sigma / I)_{\text {narrow }} /(\sigma / I)_{\text {chamber }}$, where $(\sigma / I)_{\text {narrow }}$ is the value for a droplet undergoing its first deformation in the narrow region of the microchannel and $(\sigma / I)_{\text {chamber }}$ is the "original" value of the isotropic network state of the same droplet while it resided in the reaction chamber. Comparing the calculated values of $\sigma$ with visual inspection of fluorescence images, it appears that $\sigma>2$ correlates with a segregation of fibrin-rich and fibrin-poor regions within a single droplet. Moreover, a critical 
rate of deformation $\varepsilon \approx 1 \mathrm{~s}^{-1}$ can be identified as a threshold for this coacervation behavior.

The effect of this threshold velocity is manifested as irreversible clotting, similar to that observed in the microdevice discussed earlier (Fig. 6.2). However, in the reaction chamber, the droplets initially contained an isotropic, fibrous ripened network. This is considerably different to the homogenous mixture used as the starting point in measurements of $\sigma^{*}$ in the serpentine device, where the continuously moving droplet did not allow droplet spanning network formation. The fact that a similar $\sigma$ parameter describes the aggregation phenomena in both cases demonstrates the generality of hydrodynamically induced aggregation. Therefore, these hydrodynamic effects may be a crucial consideration in droplet-based experiments, where multiple components experience microfluidic flow conditions. This effect is especially important in bionetwork formation, as shown here, and other processes such as polymerization (e.g. online particle or capsule synthesis). Furthermore, the complex effects of hydrodynamics

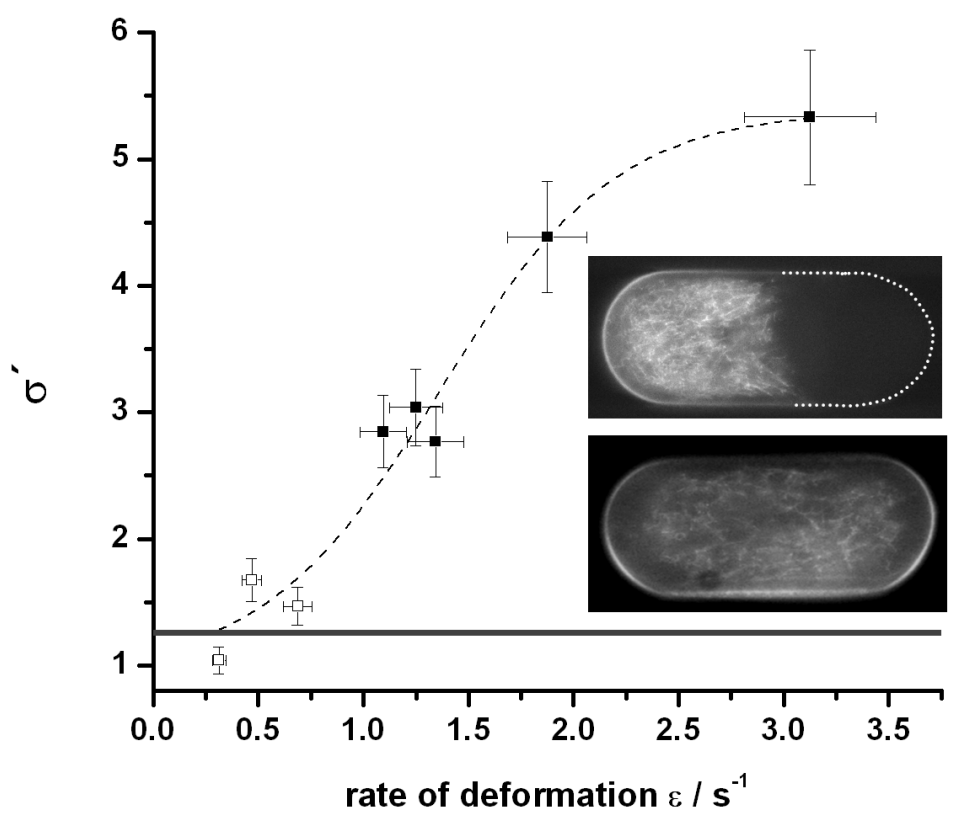

Figure 6.8: Two "phases" of fibrin-rich and fibrin-poor regions are found for droplets experiencing rates of deformation $\varepsilon$ approximately one or greater, which is reflected by their higher values of $\sigma$ (upper image). No separation is visually observed for less than one, where $\sigma<$ 2 (white data points, also see lower image). For comparison, the horizontal gray line is the average $\sigma^{*}$ value in stopped flow from Fig. 6.3. Dotted line is guide to the eye.

on network aggregation under microfluidic flow conditions might suggest a general tendency 
of fibrin networks to aggregate in vitro. The preferred aggregation states also serve as a reminder that the networks are delicate and non-equilibrium in their nature and therefore access to them, via microfluidic tools, is particularly novel.

The protein fibrin was selected to explore the influence of microfluidic flow on the formation and aggregation of a bionetwork. Fibrinogen forms a three dimensional fibrin network that is a primary component of blood clots in vivo. By its nature, fibrin exhibits strong adhesion to surfaces including microchannel walls and therefore, the networks in this study were confined within droplets. This was achieved using tailored microfluidic chips, containing sites for droplet formation, electrocoalescence, flow and quasi-static observations, and mechanical manipulation the fibrin networks. The formation of fibrin was initiated in a droplet by coalescing two droplets, one with the fibrinogen monomer and the other containing the enzyme thrombin. The development of fibrin fibers within the resulting droplet required time for activation and fibril growth, and eventually neighboring fibers interact to form a three-dimensional network due to physical and chemical inter-fiber contacts.

Hydrodynamic flow within the droplets was found to dramatically accelerate the aggregation of fibrin into small and, eventually, very large particles, i.e. clots. However, when the flow was quasi-static, isotropic three-dimensional networks formed. In this case, the fibrin formation was not influenced by extreme flow patterns because it remained "parked" in a chamber. These isotropic networks were then driven through repeated geometric confinements to study the elasticity of the networks. Fluorescence analysis of the fibrin network at low velocities did not show any evidence of inelastic deformation, despite undergoing repeated cycles of compression. However, a critical rate of deformation $\varepsilon$ was identified, above which fibrin-rich and fibrin-poor regions appear within the droplet. In this case, the clotting behavior is analogous to that found earlier for networks which form under continuous flow conditions. In both situations, the standard deviation of intensities normalized by the mean intensity, i.e. $\sigma^{*}$ or $\sigma$, is an appropriate parameter to quantify the extent of aggregation [147].

Our microfluidic approach allows the visualization of fibrin coacervation under a variety of experimental conditions. Therefore, the tendency of fibrin networks to aggregate can be approached in multiple ways to understand some general principles of the protein behavior. In the case of fibrin, its naturally "sticky" properties are conserved in the microdevices, which manifests as a form of "clotting". High quality fluorescence images allow the direct visualization of the networks and aggregates. This, in concert with precise mixing of small volumes in microfluidic devices, enables detailed studies of network behavior and conformation under confinement and well-controlled experimental conditions. Generally speaking, the powerful 
experimental approach demonstrated here is broadly relevant to a wide variety of physical systems where a greater understanding of the delicate interplay between material properties and flow is desired. 


\section{Chapter 7}

\section{Summary}

The main objective of this thesis was to study the topological transitions between the different arrangements of an emulsion e.g. by varying the geometry of the microchannel. In-situ production of a monodisperse emulsion was achieved using T-junction or step emulsification techniques. Due to surface energy minimization, the droplet arrangement was largely determined by the microchannel geometry and the droplet dimensions, while the dispersed phase volume fraction determined the packing density.

We found that droplet arrangements can be stable or metastable depending on the geometry of the confining microchannel. These arrangements were manipulated between the bamboo and zigzag arrangement or zigzag and three-row arrangement provided the normalized droplet size $A^{*}$ is close to $A^{*}=1 / \sqrt{3}$ or $A^{*}=1 / 3(1+1 / 2 \sqrt{3})^{-1}$, respectively, representing the energetic stability criterions in a $2 \mathrm{D}$ geometry. In these cases one can reversibly change between energetically meta-stable and stable arrangements. This was achieved either by static or by temporary variable geometric constrictions generated by an applied inhomogeneous magnetic field. In both cases the transition is induced purely geometrically by a tapered constriction. The range of drop size $A^{*}$, where either a bamboo, a zigzag, or three-row arrangement is accessible depends on the exact shape of the geometric constriction, e.g. the downstream angle, and the dispersed volume fraction. The temporarily generated constriction in the case of ferrofluids can be furthermore used to actively select the outgoing channel of a Y-junction.

Besides the precise geometry of the constriction, the hysteretic behavior also depends on the volume fraction of the dispersed phase. When an emulsion is flowing through the tapered channel where the channel width is continuously increasing we found a structural transition between bamboo to zigzag and zigzag to three-row arrangements. Conversely, as the channel 
continuously narrows the reverse process occurs, but at different channel widths, revealing the hysteretic behavior. This hysteresis is found to increase with the volume fraction of the dispersed phase and becomes noticeable for volume fraction $\phi>0.8$. Consequently, the probability to retain a particular droplet arrangement increases with increasing dispersed phase volume fraction. The physical reason for this hysteretic behavior is the finite energy required for topological transitions of the foam-like network. The stability of certain droplet arrangements and their transitions were studied for the simple two-dimensional model for static emulsion arrangements. By applying a lateral force into the static droplet arrangement, we could achieve the equilibrium conditions of different arrangements due to the variation of dispersed phase volume fraction during the droplet compression. We compared the experimental results with the numerical calculation and found qualitative agreement.

Furthermore, by incorporating corners into the microfluidic channel network we performed precise manipulation of the individual droplet positions with respect to the neighboring droplets. The phase shifts of the droplets were explained by considering the path length differences of the inner and outer row of droplets travelling through the corner. The number of phase shifts as a function of drop size, dispersed volume fraction and corner angles were studied quantitatively and show reasonable agreement with the theoretical consideration based on the path length differences. However, the number of phase shifts is not additive if we combine several corners of different angles. Our experimental result suggest that the phase shifts can be maintained if appropriately shaped corners are inserted along a straight channel. This may be applied to combinatorial multistep reactions where the neighbouring droplets have to be exchanged.

Finally, the techniques developed so far were applied to a biophysical problem. By isolating sticky biological species within the nanoliter volume of droplets and coalescing them online we were able to observe the evolution of an encapsulated fibrin network. The development of fibrin fibers within the droplet required time for activation and fibril growth, and eventually neighboring fibers interact to form a three-dimensional network due to physical and chemical inter-fiber contacts. Hydrodynamic flow within the droplets was found to dramatically accelerate the aggregation of fibrin into small and, eventually, very large particles, i.e. clots. However, when the droplet was parked in the reaction chamber, isotropic threedimensional networks formed. These isotropic networks were then driven through repeated geometric confinements to study the elasticity of the networks. Fluorescence analysis of the fibrin network at low velocities did not show any evidence of inelastic deformation, despite undergoing repeated cycles of compression. However, a critical velocity was identified, above 
which fibrin-rich and fibrin-poor regions appear within the droplet. In this case, the clotting behavior was analogous to that dynamic case where fibrin networks formed under continuous flow conditions. In both situations, the standard deviation of intensities normalized by the mean intensity was found to be an appropriate parameter to quantify the extent of aggregation. 



\section{Appendices}





\section{Appendix A}

\section{Symbols and notations}

\begin{tabular}{c|c|c}
\hline Symbol & Description & value $/$ unit \\
\hline \hline$R e$ & Reynolds number & - \\
$\rho$ & Density & $\mathrm{kg} / \mathrm{m}^{3}$ \\
$v$ & Velocity & $\mathrm{m} / \mathrm{s}$ \\
$\eta$ & Viscosity & $\mathrm{Pa} \cdot \mathrm{s}$ \\
$l$ & Length & $\mathrm{m}$ \\
$C a$ & Capillary number & - \\
$R$ & Radius & $\mathrm{m}$ \\
$V$ & Volume & $\mathrm{m}^{3}$ \\
$d$ & Distance & $\mathrm{m}$ \\
$D$ & Diffusion coefficient & $\mathrm{cm} / \mathrm{s}$ \\
$t$ & Time & $\mathrm{s}$ \\
$\gamma, \gamma_{0}$ & Surface tension & $\mathrm{N} / \mathrm{m}$ \\
$\gamma_{i j}$ & Interfacial tension with $\{$ i,j\} being any two mediums $\{\mathrm{S}, \mathrm{L}, \mathrm{V}\}$ & $\mathrm{N} / \mathrm{m}$ \\
$\Delta \mathrm{P}$ & Laplace pressure & $\mathrm{Pascal}$ \\
$r_{1}, r_{2}$ & Principal radii of curvatures & $\mathrm{m}$ \\
$g$ & Acceleration due to gravity & $\approx .8 \mathrm{~m} / \mathrm{s}^{2}$ \\
$\lambda$ & Wavelength & $\mathrm{m}$ \\
$\omega$ & Frequency & $\mathrm{Hz}$ \\
$U$ & Voltage & $\mathrm{V}$ \\
$\epsilon$ & Dielectric constant & - \\
$q$ & Wave number & $\mathrm{m}{ }^{-1}$ \\
$A$ & Projected area of a droplet & - \\
$W$ & Channel width & - \\
& &
\end{tabular}




\begin{tabular}{c|c|c}
$A^{*}$ & Normalized 2D area of a droplet & - \\
$\phi$ & Dispersed phase volume fraction & - \\
$E$ & Excess free energy & - \\
$\alpha$ & Corner angle & degrees \\
$\beta$ & The angle of deviation from the straight channel & - \\
$\delta$ & Phase shift & - \\
$\Delta$ & Average difference in the pathlength & - \\
$n$ & Number of rows in a droplet arrangement & $180^{\circ}$ \\
$\pi$ & Ratio of a circle's circumference to it's diameter & $\mathrm{ml} / \mathrm{h}$ \\
$Q_{t}$ & Total flow rate & $\mathrm{ml} / \mathrm{h}$ \\
$Q_{d}$ & Dispersed phase flow rate & $\mathrm{ml} / \mathrm{h}$ \\
$Q_{c}$ & Continuous phase flow rate & Pascal $\left(\mathrm{N} / \mathrm{m}^{2}\right)$ \\
$P$ & Pressure & $\mathrm{kg} \cdot \mathrm{m} / \mathrm{s}^{2}$ \\
$F$ & Force & $\mathrm{s}$ \\
$\varepsilon$ & Rates of deformation & - \\
$\sigma^{*}$ & The normalized standard deviation of grayscale intensities & - \\
$I$ & Mean intensity & -
\end{tabular}




\section{Appendix B}

\section{Recipe for photolithographic devices}

\section{B.1 Underlayer}

1. Rinse round glass slides with isopropanol (2-propanol), dry in nitrogen stream

2. Bake at $200^{\circ} \mathrm{C}$ for $3-5$ minutes

3. Spincoat OMNICOAT at $3000 \mathrm{rpm}$

4. Bake at $200^{\circ} \mathrm{C}$ for $>2 \mathrm{~min}$

5. Cool hotplate to $95^{\circ} \mathrm{C}$ (WAIT before continuing)

6. Spincoat SU8-2005 at $5000 \mathrm{rpm}$

7. Bake at $65^{\circ} \mathrm{C}$ for $1 \mathrm{~min}$

8. Bake at $95^{\circ} \mathrm{C}$ for $3 \mathrm{~min}$

9. Expose entire wafer (ie. no mask, soft contact) at $15 \mathrm{~mW} / \mathrm{cm}^{2}$ for $20 \mathrm{sec}$

10. Bake at $65^{\circ} \mathrm{C}$ for $1 \mathrm{~min}$

11. Bake at $95^{\circ} \mathrm{C}$ for $1 \mathrm{~min}$

12. Plasma on 'HI' for $30 \mathrm{sec}$ (samples upside down to avoid dust settling on layer).

\section{B.2 Channel structures}

\section{B.2.1 Resist Structure: $\mathbf{3 0} \mu \mathrm{m}$ channel height}

1. Spincoat SU8-50 at $2000 \mathrm{rpm}$

2. Soft bake at $65^{\circ} \mathrm{C}$ for $4 \mathrm{~min}$

3. Soft bake at $95^{\circ} \mathrm{C}$ for $13 \mathrm{~min}$ 
4. Expose (through mask, HP contact mode) at $15 \mathrm{~mW} / \mathrm{cm}^{2}$ for $30 \mathrm{sec}$

5. Post expose bake at $65^{\circ} \mathrm{C}$ for $1 \mathrm{~min}$

6. Post expose bake at $95^{\circ} \mathrm{C}$ for $2 \mathrm{~min}$

7. Develop for 2 min in SU8 developer on mechanical shaker.

\section{B.2.2 Resist Structure: $\mathbf{5 0} \mu \mathrm{m}$ channel height}

1. Spincoat SU8-50 at $1500 \mathrm{rpm}$

2. Soft bake at $65^{\circ} \mathrm{C}$ for $5 \mathrm{~min}$

3. Soft bake at $95^{\circ} \mathrm{C}$ for $15 \mathrm{~min}$

4. Expose (through mask, HP contact mode) at $15 \mathrm{~mW} / \mathrm{cm}^{2}$ for $30 \mathrm{sec}$

5. Post expose bake at $65^{\circ} \mathrm{C}$ for $1 \mathrm{~min}$

6. Post expose bake at $95^{\circ} \mathrm{C}$ for $2 \mathrm{~min}$

7. Develop for 5 min in SU8 developer on mechanical shaker.

\section{B.2.3 Resist Structure: $80 \mu \mathrm{m}$ channel height}

1. Spincoat SU8-100 at $2000 \mathrm{rpm}$

2. Soft bake at $65^{\circ} \mathrm{C}$ for $10 \mathrm{~min}$

3. Soft bake at $95^{\circ} \mathrm{C}$ for $30 \mathrm{~min}$

4. Expose (through mask, HP contact mode) at $15 \mathrm{~mW} / \mathrm{cm}^{2}$ for $30 \mathrm{sec}$

5. Post expose bake at $65^{\circ} \mathrm{C}$ for $1 \mathrm{~min}$

6. Post expose bake at $95^{\circ} \mathrm{C}$ for $5 \mathrm{~min}$

7. Develop for $5 \mathrm{~min}$ in SU8 developer on mechanical shaker.

\section{B.2.4 Resist Structure: $120 \mu \mathrm{m}$ channel height}

1. Spincoat SU8-100 at $1700 \mathrm{rpm}$

2. Soft bake at $65^{\circ} \mathrm{C}$ for $13 \mathrm{~min}$

3. Soft bake at $95^{\circ} \mathrm{C}$ for $37 \mathrm{~min}$

4. Expose (through mask, HP contact mode) at $15 \mathrm{~mW} / \mathrm{cm}^{2}$ for $30 \mathrm{sec}$

5. Post expose bake at $65^{\circ} \mathrm{C}$ for $1 \mathrm{~min}$

6. Post expose bake at $95^{\circ} \mathrm{C}$ for $7 \mathrm{~min}$

7. Develop for 8 min in SU8 developer on mechanical shaker. 


\section{B.3 Preparation of PMMA coated cover plate}

1. Rinse round glass slides with isopropanol (2-propanol), dry in nitrogen stream

2. Bake at $180^{\circ} \mathrm{C}$ for $3-5$ minutes

3. If needed, gold electrodes were thermally vapor deposited at $10^{-6}$ mbar onto the cover plate, with an underlying chromium adhesion layer.

4. Prepare the wires for electrode using CW2400 Conductive Epoxy Glue (ITW Chemtronics ${ }^{\circledR}$ )

5. Spincoat PMMA Photoresist (A11) at $1500 \mathrm{rpm}$

6. Bake at $180^{\circ} \mathrm{C}$ for $>2 \min$

7. Check layer to ensure NO bubbles etc...

\section{B.4 Thermal bonding of the channels and the cover plate}

1. Drill the holes for the tubing connections through the supporting glass substrate

2. Clean the channels by nitrogen stream

3. Bond the cover plate coated with PMMA and the channel system at $160^{\circ}$ at the thermal bonding device by applying the pressure (max 0.6 bar) 2-3 times repeatedly to achieve a good bonding.

\section{B.5 Nanoport bonding}

1. Prepare the bonding surfaces of both the Port and the microdevice by cleaning with isopropanol

2. Bond the Nanoport Flat Bottom Port to the microdevice using heat activated adhesive film tape (FST 3M 583)

3. Clamp the Port to the microdevice. Place clamped Ports in oven at $120^{\circ}$ for 90 mins to develop a complete bonding

4. Connect the microchannel with teflon tubing using Nanoport Nut FT PEEK and Ferrule. 



\section{References}

[1] Reyes, D. R.; Iossifidis, D.; Auroux, P. A.; Manz, A. Anal. Chem. 2002, 74, 2623.

[2] Atencia, J.; Beebe, D. J. Nature 2005, 437, 648.

[3] Teh, S.; Lin, R.; Hung, L.; Lee, A. P. Lab Chip 2002, 8, 198.

[4] Joanicot, M.; Ajdari, A. Science 2005, 309, 887.

[5] Liau, A.; Karnik, R.; Majumdar, A.; Doudna Cate, J. H. Anal. Chem. 2005, 77, 7618.

[6] Stone, Z. B.; Stone, H. A. Phys. Fluids 2005, 17, 063103.

[7] Tanthapanichakoon, W.; Aoki, N.; Matsuyama, K.; Mae, K. Chem. Eng. Sci. 2006, 61, 4220.

[8] Grigoriev, R. O.; Schatz, M. F.; Sharma, V. Lab Chip 2006, 6, 1369.

[9] Sarrazin F.; Loubiere, K.; Prat, L.; Gourdon, C.; Bonometti, T.; Magnaudet, J. AIChE 2006, 52, 4061.

[10] Chen, D. L.; Li, L:; Reyes, S.; Adamson, D. N.; Ismagilov, R. F. Langmuir 2007, 23, 2255 .

[11] Thorsen, T.; Maerkl, S. J.; Quake, S. R. Science 2002, 298, 580.

[12] Kashid, M. N.; Gerlach, I.; Goetz, S.; Franzke, J.; Acker, J. F.; Platte, F.; Agar, D. W.; Turek, S. Ind. Eng. Chem. Res. 2005, 44, 5003.

[13] Kinoshita, H.; Kaneda, S.; Fujii, T.; Oshima, M. Lab Chip 2007, 7,338. 
[14] Malsch, D.; Kielpinski, M.; Merthen, R.; Albert, J.; Mayer, G.; Köhler, J. M.; Süsse, H.; Stahl, M.; Henkel, T. Chem. Eng. J. 2008, 135S, S166.

[15] Eckhardt, B.; Hascoet, E. Phys. Rev. E 2005, 72, 037301.

[16] Oddy, M. H.; Santiago, J. G.; Mikkelsen, J. C. Anal. Chem. 2001, 73, 5822.

[17] Stroock, A. D.; et al., Science 2002, 295, 647.

[18] Solomon, T. H.; Mezic, I. Nature 2003, 425, 376.

[19] Stone, H. A.; Stroock, A. D.; Ajdari, A. Ann. Rev. Fluid Mech. 2004, 36, 381.

[20] Tabeling, P.; et al., Phil. Trans. Roy. Soc A 2004, 362, 987.

[21] Nguyen, N.-T.; Wu, Z. J. Micromech. Microeng. 2005, 15, R1.

[22] Song, H.; Tice, J. D.; Ismagilov, R. F. Angew. Chem. Int. Ed. 2003, 42, 767.

[23] Li, L.; Boedicker, J. Q.; Ismagilov, R. F. Anal. Chem. 2007, 79, 2756.

[24] Song, H.; Ismagilov, R. F. J. Am. Chem. Soc. 2003, 125, 14613.

[25] Chen, D. L.; Gerdts, C. J.; Ismagilov, R. F. J. Am. Chem. Soc. 2005, 127, 9672.

[26] Song, H.; Chen, D. L.; Ismagilov, R. F. Angew. Chem. Int. Ed. 2006, 45, 7336.

[27] Song, H.; Li, H. W.; Munson, M. S.; Van Ha, T. G.; Ismagilov, R. F. Anal. Chem. 2006, $78,4839$.

[28] Köhler J. M.; et al., Chem. Eng. J. 2004, 101, 201.

[29] Zheng, B.; Roach, L. S.; Ismagilov, R. F. J. Am. Chem. Soc. 2003, 125, 11170.

[30] Zheng, B.; Tice, J. D.; Ismagilov, R. F. Anal. Chem. 2004, 76, 4977.

[31] He, M.; Edgar, J. S.; Jeffries, G. D. M.; Lorenz, R. M.; Shelby, J. P.; Chiu, D. T. Anal. Chem. 2005, 77, 1539.

[32] Linder, V.; Sia, S. K.; Whitesides, G. M. Anal. Chem. 2005, 77, 64.

[33] Hatakeyama, T.; Chen, D. L.; Ismagilov, R. F. J. Am. Chem. Soc. 2006, 126, 2518. 
[34] Adamson, D. N.; Mustafi, D.; Zhang, J. X. J.; Zheng, B.; Ismagilov, R. F. Lab Chip 2006, 6, 1178.

[35] Chen, D. L.; Ismagilov, R. F. Curr. Opin. Chem. Biol. 2006, 10, 226.

[36] Priest, C.; Herminghaus, S.; Seemann, R. Appl. Phys. Lett. 2006, 13, 134101.

[37] Fair, R. B. Microfluidics Nanofluidics 2007, 3,245.

[38] Link, D. R.; Grasland-Mongrain, E.; Duri, A.; Sarrazin, F.; Cheng, Z.; Cristobal, G.; Marquez, M.; Weitz, D. A. Angew. Chem. Int. Ed. 2006 45, 2556.

[39] Lau, B. T. C.; Baitz, C. A.; Dong, X. P.; Hansen, C. L. J. Am. Chem. Soc. 2007, 129, 454.

[40] Thorsen, T.; Roberts, R. W.; Arnold, F. H.; Quake, S. R. Phys. Rev. Lett. 2001, 86, 4163.

[41] Nisisako, T.; Torii, T.; Higuchi, T. Lab Chip 2002, 2, 24.

[42] Anna, L.; Bontoux, N.; Stone, H. A. Appl. Phys. Lett. 2003, 82, 364.

[43] Gañan-Calvo, A. M.; Gordillo, J. M. Phys. Rev. Lett 2001, 87, 274501.

[44] Priest, C.; Herminghaus, S.; Seemann, R. Appl. Phys. Lett. 2006, 88, 024106.

[45] Priest, C.; Surenjav, E.; Herminghaus, S.; Seemann, R. Proc. 4th World Congress on Emulsions 2006.

[46] Nguyen, N.; Lassemono, S.; Chollet, F. A. Sens. Actuators, B 2006, 117, 431.

[47] Niu, X.; Zhang, M.; Peng, S.; Wen, W.; Sheng, P. Biomicrofluidics 2007, 1, 044101.

[48] Velev, O. D.; Prevo, B.G.; Bhatt, K. H. Nature 2003, 426515.

[49] Engl, W.; Roche, M.; Colin, A.; Panizza, P.; Ajdari, A. Phys. Rev. Lett. 2005, 95, 208304.

[50] Ahn, K.; Kerbage, C.; Hunt, T.; Westervelt, R. M.; Link, D. R.; Weitz, D. A. Appl. Phys. Lett. 2006, 88, 024104.

[51] Tan, Y.C.; Fisher, J. S.; Lee, A. I.; Christini, V.; Lee, A. P. Lab Chip 2004, 4, 292. 
[52] Niu, X.; Gulati, S.; Edel, J. B.; deMello, A. J. Lab Chip 2008, 8, 1837.

[53] Kralj, J. G.; Schmidt, M. A.; Jensen, K. F. Lab Chip 2005, 5, 531.

[54] Chabert, M.; Dorfman, K. D.; Viovy, J. L. Electrophoresis, 2005, 26, 3706.

[55] Mullis, K. B.; Ferré, F.; Gibbs, R. A. Birkhäuser, Boston 1994.

[56] Sugiura, S.; Odad, T.; Izumidaa, Y.; Aoyagid, Y.; Satakef, M.; Ochiaie, A.; Ohkohchid, N.; Nakajimaa, M. Biomaterials 2005, 26, 3327.

[57] Barbulovic-Nad, I.; Philip, H. Y.; Parkc, S.; Wheeler, A. R. Lab Chip 2008, 8, 519.

[58] Hansen, C. L.; Classen, S.; Berger, J. M.; Quake, S. R. J. Am. Chem. Soc. 2006, 128, 3142 .

[59] Xu, S.; Nie, Z.; Seo, M.; Lewis, P.; Kumacheva, E.; Stone, H. A. Garstecki, P.; Weibel, D. B.; Gitlin, I.; Whitesides, G. M. Angew. Chem. Int. Ed. 2005, 44, 724.

[60] Kim, J.; Utada, A. S.; Fernández-Nieves, A.; Hu, Z.; Weitz, D. A. Angew. Chem. Int. Ed. 2007, 46, 1819.

[61] Demirci, U.; Montesano, G. Lab Chip 2007, 7, 1139.

[62] Hansen, C.; and Quake, S. R. Curr. Opin. Str. Bio. 2003, 13, 538.

[63] Sia, S. K.; Whitesides, G. M. Electrophoresis 2003, 24, 3563.

[64] Hirano, M.; Torii, T.; Higuchi, T.; Kobayashi, M.; Yamazaki, H. The Proceedings of the $\mu$ TAS 2003 2003, 1, 473.

[65] Hansen, F. K.; Rodsrun, G. J. Coll. Inter. Sci. 1991, 141, 1.

[66] Landfester, K. Macromol. Symp. 2000, 150, 171.

[67] El-Aasser, M. S.; Sudol, E. D. J. Coat. Tech. Res. 2004, 1, 21.

[68] Goodwin, G. W. Colloids and Interfaces with Surfactants and Polymers - An Introduction 2004.

[69] Pickering, S. U. J. Chem. Soc. 1907, 91, 2001. 
[70] Bancroft, W. D. J. Phys. Chem. 1913, 17, 501.

[71] Griffin, W. C. J. Society of Cosmetic Chemists 1949, 311.

[72] Schramm, L. L. Emulsions, Foams, and Suspensions Fundamentals and Applications 2005.

[73] Krieger, M.; Dougherty, T. G. Trans. Soc. Rheol. 1959, 3, 137.

[74] Nakashima, T.; Shimizu, M.; Kukizaki, M. Key Eng. Mater. 1991, 61, 513.

[75] Abrahamse, A. J.; van der Padt, A.; Boom, R. M.; de Heij, W. B. C. AIChE J. 2001, 47, 1285.

[76] Gañan-Calvo, A. M. Phys. Rev. Lett. 1998, 80, 285.

[77] Mason, T. G.; Bibette, J. Langmuir 1997, 13, 4600.

[78] Perrin, P. Langmuir 1998, 14, 5977.

[79] Sugiura, S.; Nakajima, M.; Seki, M. Langmuir 2002, 18, 3854.

[80] Dreyfus, R.; Tabeling, P.; Willaime, H. Phys. Rev. Lett. 2003, 90, 144505.

[81] Seo, M.; Paquet, C.; Nie, Z.; Xu, S.; Kumacheva, E. Soft Matter 2007, 3, 986.

[82] Okushima, S.; Nisisako, T.; Torii, T.; Higuchi, T. Langmuir 2004, 20, 9905.

[83] Utada, A. S.; Lorenceau, E.; Link, D. R.; Karlon, D. P.; Stone, H. A.; Weitz, D. A. Science 2005, 308, 537.

[84] Nisisako, T. Chem. Eng. Technol. 2008, 31, 1091.

[85] Muschiolik, G. Curr. Opin. Colloid Interface Sci. 2007, 12, 213.

[86] Miyazawa, K.; Yajima, I.; Kaneda, I.; Yanaki, T. J. Cosmet. Sci. 2000, 51, 239.

[87] Davis, S. S.; Walker, I. M. Methods Enzymol. 1987, 149, 51.

[88] Taylor, P. Adv. Coll. Inter. Sci. 1998, 75, 107.

[89] Mun, S.; McClements, D. J. Langmuir 2006, 22, 1551. 
[90] Weaire, D.; Hutzler, S. The Physics of Foams 2001, Clarendon, Oxford.

[91] During the T1 process, the initial local configuration of the lamellae, as described by an "H", approaches an unstable "X" and separates spontaneously to an "H" rotated by an angle of $90^{\circ}$.

[92] Durand, M.; Stone, H. A. Phys. Rev.Lett. 2006, 97, 226101.

[93] Weaire, D.; Vaz, M. F.; Teixeira, P. I. C.; Fortes, M. A. Soft Matter 2007, 3, 47.

[94] Drenckhan, W.; Hutzler, S.; Weaire, D. Modern Trends in Physics Research 2005, 748, 22.

[95] Rosensweig, R. E. Ferrohydrodynamics 1997, Dover publications, inc, New York.

[96] Pollack, M. G.; Shenderov, A. D.; Fair, R. B. Lab Chip 2002, 2, 96.

[97] Feng, R.; Farris, R. J. J. Micromech. Microeng. 2003, 13, 80.

[98] Gunther, A.; Khan, S. A.; Thalmann, M.; Trachsel, F.; Jensen, K. F. Lab Chip 2004, 4, 278.

[99] Zheng, B.; Tice, J. D.; Roach, L. S.; Ismagilov, R. F. Angew. Chem. Int. Ed. 2004, 43, 2508.

[100] Zheng, B.; Ismagilov, R. F. Angew. Chem. Int. Ed. 2005, 44, 2520.

[101] Garstecki, P.; Fuerstman, M. J.; Stone, H.; Whitesides, G. M. Lab Chip 2006, 6, 437.

[102] Lorenceau, E.; Utada, A. S.; Link, D. R.; Cristobal, G.; Joanicot, M.; Weitz, D. A. Langmuir 2005, 21, 9183.

[103] Gañan-Calvo, A. M.; Martin-Banderas, L.; Gonzalez- Prieto, R.; Rodriguez-Gil, A.; Berdun-Alvarez, T.; Cebolla, A.; Chavez, S.; Flores-Mosquera, M. Int. Jour. Pharm 2006, 324, 19.

[104] Schneider, T.; Zhao, H.; Jackson, J. K.; Chapman, G. H.; Dykes, J.; Haefeli, U.O. published in J. Phar. Sci..

[105] Link, D. R.; Anna, S. L.; Weitz, D. A.; Stone, H. A. Phys. Rev. Lett. 2004, 92, 054503. 
[106] Chokkalingam, V.; Herminghaus, S.; Seemann, R. Submitted to Appl. Phys. Lett..

[107] Chokkalingam, V.; Priest, C.; Krämer, M.; Maier, W. F.; Herminghaus, S.; Seemann, R. The Proceedings of the $\mu$ TAS 2007, Eds. J.-L. Viovy, P. Tabeling, S. Descroix, L. Malaquin, ISBN 978-0-9798064-0-7.

[108] Bremond, N., Thiam, A. R., Bibette, J. Phys. Rev. Lett. 2008, 100, 024501.

[109] Fidalgo, L. M., Abell, C., Huck, W. T. S. Lab Chip 2007, 7, 984.

[110] Herminghaus, S. Phys. Rev. Lett. 2007, 83, 2359.

[111] Weibel, D. B.; Krulthof, M.; Potenta, S.; Sla, S. K.; Lee, A.; Whitesides, G. M. Anal. Chem. 2005, 77, 4726.

[112] Drenckhan, W.; Cox, S. J.; Delaney, G.; Holste, H.; Weaire, D.; Kern, N. Coll. Surf. A 2005, 263, 52 .

[113] Solans, C.; Pons, R.; Kunieda, H. Modern Aspects of Emulsion Science 1999, book chapter 11, 366-394.

[114] Hutzler, S.; Peron, N.; Weaire, D.; Drenckhan, W. Eur. Phys. J. E 2004,14,381.

[115] Hutzler, S.; Weaire, D.; Elias, F.; Janiaud, E. Phil. Mag. Lett. 2002, 82, 297.

[116] Elias, F.; Bacri, J. C.; Flament, C.; Janiaud, E.; Talbot, D.; Drenckhan, W.; Hutzler, S.; Weaire, D. Coll. Surf. A 2005,263,65.

[117] Surenjav, E.; Evans, H.; Pfohl, T.; Priest, C.; Herminghaus, S.; Seemann, R. The Proceedings of the $\mu$ TAS 2007, Eds. J.-L. Viovy, P. Tabeling, S. Descroix, L. Malaquin, ISBN 978-0-9798064-0-7, 339.

[118] Surenjav, E.; Priest, C.; Herminghaus, S.; Seemann, R. Lab Chip 2009, DOI: 10.1039/B808160C.

[119] Strictly speaking, the excess free energy per unit area of a lamella is $2 \gamma+F(h)$, where $h$ is the lamella thickness and $F(h)$ is the effective interface potential. The latter comes into play when the lamellae have time to thin down to molecular dimensions. This is probably not of great importance in our dynamic system. We neglect $F(h)$ throughout. 
[120] Füredi, Z. Discrete Comput. Geom. 1991, 6, 95.

[121] Priest, C.; Surenjav, E.; Herminghaus, S.; Seemann, R. The Proceedings of the $\mu$ TAS 2007, Eds. J.-L. Viovy, P. Tabeling, S. Descroix, L. Malaquin, ISBN 978-0-9798064-07,931 .

[122] Raj, K.; Moskowitz, B.; Casciari, R. J. Magn. Magn. Mater. 1995, 149, 174.

[123] Pamme, N. Lab Chip 2006, 6, 24.

[124] Hatch, A.; Kamholz, A. E.; Holman, G.; Yager, P.; Bohringer, K. F. J. Microelectromech. Syst. 2001, 10, 215.

[125] Hartshorne, H.; Backhouse, C. J.; Lee, W. E. Sens. Actuators, B 2004, 99, 592.

[126] Shinkai, M. J. Biosci. Bioeng. 2002, 94, 606.

[127] Pankhurst, Q. A.; Connolly, J.; Jones, S. K.; Dobson, J. J. Phys. D: Appl. Phys. 2003, $36,167$.

[128] Kern, N.; Weaire, D. Philos. Mag. 2003, 83, 2973.

[129] Höhler, R.; Sang, Y. Y. C.; Lorenceau, E.; Cohen-Addad, S. published in Langmuir 2007.

[130] Brinkmann, M.; Surenjav, E.; Seemann, R.; Herminghaus, S. in preparation.

[131] Ratner, B. Biomat. Sci, Intro. Mat. Med. Academic Press, 1996.

[132] Stormorken, H.; Sakariassen, K. S. Thrombosis Research 1997, 88, 1.

[133] Levi, M.; Keller, T. T.; Gorp, E. V.; Cate, H. T. Cardiovascular Research 2003, 60, 26.

[134] Adams, R. A.; Passino, M.; Sachs, B. D.; Nuriel, T.; Akassoglou, K. Mol. Interv. 2004, $4,163$.

[135] Rickles, F. R.; Patierno, S.; Fernandez, P. M. Chest 2003, 124, 58.

[136] Liu, W.; Jawerth, L. M.; Sparks, E. A.; Falvo, M. R.; Hantgan, R. R.; Superfine, R.; Lord, S. T.; Guthold, M. Science 2006, 313, 634.

[137] Stasio, E. D.; Nagaswami, C.; Weisel, J. W.; Cera, E. D. Biophys. J. 1998, 75, 1973. 
[138] Ferri, F.; Greco, M.; Arcovito, G.; Spirito, M. D.; Rocco, M. Phys. Rev. E 2002, 66, 011913.

[139] Ferri, F.; Greco, M.; Arcovito, G.; Bassi, F. A.; Spirito, M. D.; Paganini, E.; Rocco, M. Phys. Rev. E 2001, 63, 031401.

[140] Weisel, J. W.; David, a. J. M. S.; Parry, A. D. in Advances in Protein Chemistry Academic Press, Editon edn., 2005, 70, 247.

[141] Carr, M. E.; Hermans, J. Macromolecules 1978, 11, 46.

[142] Ryan, E. A.; Mockros, L. F.; Weisel, J. W.; Lorand, L. Biophys. J. 1999, 77, 2813.

[143] Voter, W. A.; Lucaveche, C.; Blaurock, A. E.; Erickson, H. P. Biopolymers 1986, 25, 2359.

[144] Weisel, J. W. Biophys. Chem. 2004, 112, 267.

[145] Song, H.; Bringer, M. R.; Tice, J. D.; Gerdts, C. J.; Ismagilov R. F. App. Phys. Lett. 2003, 83, 4664 .

[146] deKruif, C. G.; Weinbreck, F.; Vries, R. Curr. Opin. Coll. Int. Sci. 2004, 9, 340.

[147] Evans, H.; Surenjav, E.; Priest, C.; Herminghaus, S.; Seemann, R.; Pfohl, T. Submitted to Lab Chip. 



\section{Acknowledgments}

I thank Prof. Dr. Stephan Herminghaus for the provision of my $\mathrm{PhD}$ candidature at the Max Planck Institute for Dynamics and Self-Organization. I am gratefully thanks for the MaxPlanck Society for providing me all the accessible possibilities to conduct my research under this institution.

Particular thanks go to Prof. Dr. Ralf Seemann for his supervision during my Ph.D project. His enormous support, stimulating suggestions and encouragement helped me in every time of doing the research and writing of this thesis.

I wish to thank Dr. Craig Priest for his help and confidence especially in the early stage of my Ph.D project and also his continuous support from Australia after his return. I would also like to thank Dr. Heather Evans and Dr. Thomas Pfohl for our successful collaboration on fibrin network project and Dr. Martin Brinkmann for his contributions to my thesis on the numerical calculations of 2D emulsion instability.

I thank my colleagues Konstantina, Shashi, Julia, Venkat, Mario, Somnath, Dr. Krishnaharya, Dr. Audrey Steinberger, Dr. Zeina Khan and Dr. Yasutaka Iwashita for their help and support while my stay in Göttingen. I really enjoyed a lot to live in the environment of all well motivated people that were always supportive.

I want to thank to my friends from Göttingen, the family of Dr. Davaasambuu Jav, Byambadorj, Ganpurev, Oyunsanaa, Dr. Markus Hauck and Matthias, Dr. Byambajav Buyandelger, Odgerel, Khaliunaa for their accompany during my stay in Göttingen.

I would like to give a special thanks to my husband Undrakh and my sweet son Irmuun whose patient love and encouragement enabled me to complete this work. I also want to thank my family in Mongolia, my parents and my brothers for their endless love towards me. 



\section{Publications}

1. C. Priest, E. Surenjav, S. Herminghaus, R. Seemann, 'Gel emulsions for microfluidic processing', Proc. 4th World Congress on Emulsions, (2006).

2. C. Priest, E. Surenjav, S. Herminghaus, R. Seemann, 'Droplet-Based Microfluidics at high Dispersed-Phase Volume Fraction', Proc. muTAS2007 Vol. 1, pp. $931-933$ (2007) ISBN: 978-0-9798064-0-7.

3. E. Surenjav, H. Evans, T. Pfohl, C. Priest, S. Herminghaus, R. Seemann, 'Manipulation of Monodisperse Gel Emulsions in Microchannels', Proc. тиTAS2007, Vol. 1, pp. 339-342 (2007) ISBN: 978-0-9798064-0-7.

4. E. Surenjav, C. Priest, S. Herminghaus, R. Seemann, 'Manipulation of gel emulsions by variable microchannel geometry' Lab Chip, (2009) 9, 325.

5. H. Evans, E. Surenjav, C. Priest, S. Herminghaus, R. Seemann, T. Pfohl, 'In Situ formation, manipulation, and imaging of droplet-encapsulated fibrin networks' (submitted to Lab Chip).

6. E. Surenjav, C. Priest, S. Herminghaus, R. Seemann, 'Droplet reorganization at a corner' (in preparation).

7. M. Brinkmann, E. Surenjav, R. Seemann, S. Herminghaus, 'Stability of $2 D$ emulsion topology' (in preparation). 



\title{
Lebenslauf
}

\section{Persönlicher Status}

\author{
Name : $\quad$ Enkhtuul Surenjav \\ Geburtsdatum : $\quad$ 4. March 1979 \\ Geburtsort : $\quad$ Uws Provinz, Mongolei \\ Wohnort : $\quad$ Albrecht-Thaer Weg 8b, 37075 Göttingen \\ Staatsangehörigkeit : Mongolisch
}

\section{Ausbildung}

2006 - 2008 Promotionsstudium Physik an der Georg-August-Universität Göttingen.

2005 - 2008 Promotion am Max-Planck-Institut für Dynamik und Selbstorganisation, Göttingen.

$2000-2005$ Wiss. Mitarbeiter am Institut für Chemie und Chemische Technologie, MAS, Ulaanbaatar, Mongolei.

2002 - 2003 Wiss. Mitarbeiter an der Fakultät für Chemie und Chemische Industrie, Universität Genua, Italien.

$2000-2002$ Masterarbeit in Chemischer Technologie an der Universität Ulaanbaatar, Mongolei.

1996 - 2000 Bachelor in Chemie und Chemische Technologie an der Universität Ulaanbaatar, Mongolei. 